\title{
A Broad Class of Conservative Numerical Methods for Dispersive Wave Equations
}

\author{
Hendrik Ranocha ${ }^{* 1}$, Dimitrios Mitsotakis ${ }^{\dagger 2}$, and David I. Ketcheson ${ }^{\ddagger 1}$ \\ ${ }^{1}$ King Abdullah University of Science and Technology (KAUST), \\ Computer Electrical and Mathematical Science and Engineering Division \\ (CEMSE), Thuwal, 23955-6900, Saudi Arabia \\ ${ }^{2}$ School of Mathematics and Statistics, Victoria University of Wellington, \\ Wellington 6140, New Zealand
}

September 14, 2020

\begin{abstract}
We develop a general framework for designing conservative numerical methods based on summation by parts operators and split forms in space, combined with relaxation Runge-Kutta methods in time. We apply this framework to create new classes of fully-discrete conservative methods for several nonlinear dispersive wave equations: Benjamin-Bona-Mahony (BBM), Fornberg-Whitham, Camassa-Holm, Degasperis-Procesi, Holm-Hone, and the BBM-BBM system. These full discretizations conserve all linear invariants and one nonlinear invariant for each system. The spatial semidiscretizations include finite difference, spectral collocation, and both discontinuous and continuous finite element methods. The time discretization is essentially explicit, using relaxation Runge-Kutta methods. We implement some specific schemes from among the derived classes, and demonstrate their favorable properties through numerical tests.
\end{abstract}

Key words. invariant conservation, summation by parts, finite difference methods, spectral collocation methods, continuous Galerkin methods, discontinuous Galerkin methods, relaxation schemes

AMS subject classification. 65M12, 65M70, 65M06, 65M60, 65M20, 35Q35

\section{Introduction}

In this work we study and develop numerical discretizations for nonlinear dispersive wave equations. One of the most important features of such equations is the existence of nonlinear invariants. In addition to the total mass, many dispersive wave models possess other invariants that may represent the energy or another important physical quantity. Perhaps the most interesting feature of these systems, related to the presence of conserved quantities, is the existence of solitary wave solutions. For non-integrable systems, numerical methods are an essential tool for studying solitary waves; even for integrable systems, numerical methods are very useful for exploring solution

${ }^{*}$ ORCID: 0000-0002-3456-2277

${ }^{\dagger}$ ORCID: 0000-0003-2700-6093

†ORCID: 0000-0002-1212-126X 
behavior [5]. Both analysis and numerical experiments have demonstrated that such studies are best undertaken using numerical methods that exactly preserve the invariants of the system in question $[8,35]$. Specifically, conservative methods possess discrete solitary wave solutions that accurately approximate the true solitary waves, with an amplitude that is constant in time and a phase error that grows linearly in time [30]. In contrast, non-conservative methods typically yield discrete solutions with amplitude errors that grow linearly in time and (therefore) phase errors that grow quadratically in time. Conservative methods are thus especially desirable for conducting studies of solitary wave properties such as speed-amplitude relationships and solitary wave interactions [35], and for long-time simulations. At the same time, discrete conservation properties can be useful for proving numerical stability.

Significant work has been devoted to the development of conservative methods for certain nonlinear dispersive wave equations [13, 19, 20, 33-36, 92, 93, 103, 110-112]. Nevertheless, and despite their known advantages, conservative fully-discrete schemes are not widely available for many important dispersive nonlinear wave equations, and most methods being proposed and used are non-conservative; see e.g. [4, 5, 14, 17, 37-39, 76, 99, 100, 104]. Indeed, the development of accurate and stable schemes (even without nonlinear invariant conservation) is a challenging task and often requires the application of implicit time discretizations [16, 38, 109].

Usually, proving the conservation of invariants of dispersive partial differential equations (PDEs) at the continuous level requires application of the product/chain rule and integration by parts. To mimic this procedure at the semidiscrete level (discrete in space, continuous in time), summation by parts (SBP) operators are used, which provide a discrete analogue of integration by parts. A review of the relevant theory can be found in [28, 41,95]. Nowadays, many different schemes have been formulated in the SBP framework, e.g. finite difference [94], finite volume [73, 74], discontinuous Galerkin [45], and flux reconstruction methods [88]. At internal interfaces or external boundaries, SBP methods can be combined with a weak imposition of interface/boundary conditions using so-called simultaneous approximation terms (SATs) to bound the energy of the semidiscretization $[23,24]$.

Since the chain and product rules cannot hold discretely for many high-order discretizations [82], split forms that preserve local conservation laws are used; cf. [43]. These are related to entropy-conservative methods in the sense of Tadmor $[42,60,79,96]$. Although the idea to use split forms is not exactly new [90, eq. (6.40)], it is still state of the art and enables the construction of numerical methods with desirable properties [46]. Conservative discretizations based on classical finite element methods require the exact integration of nonlinear terms, which can become very costly or even impossible for non-polynomial nonlinearities. Conservative methods based on split forms do not require exact integration, so they can both be cheaper and result in better stability properties [102].

All of the previously existing conservative numerical methods such as $[50,63,64,105,111]$ are constructed using ad hoc techniques tailored specifically to both the equation and the numerical method. Typically, the ideas used to construct one such scheme cannot immediately be adapted to another equation or type of discretization. In contrast, we propose a unifying spatial discretization framework based on SBP operators. We first establish general technical results and then apply these to concrete physical models, obtaining a set of necessary algebraic conditions for conservative semidiscretizations. These conditions can be satisfied by numerical schemes from any of the classes included in the unifying SBP framework.

To transfer the semidiscrete conservation results to fully-discrete schemes, the recent relaxation approach is used [53, 83-85, 89]. First ideas for such techniques date back to [92, 93] and [32, pp. 265-266] but have been developed widely just recently.

The numerical methods developed and studied in this article are implemented in Julia [12], using the time integration schemes of DifferentialEquations.j1 [78] and Matplotlib for the plots [51]. The source code for all numerical methods and the experiments is available online [86].

This article is structured as follows. Firstly, the concept of SBP operators is recalled in Section 2 and some technical results are provided that will be applied later to prove the discrete conservation 
properties. Afterwards, the relaxation approach in time is briefly summarized in Section 3 . Having established the framework of numerical methods, we concentrate on the Benjamin-Bona-Mahony (Section 4.1), Fornberg-Whitham (Section 4.2), Camassa-Holm (Section 4.3), Degasperis-Procesi (Section 4.4), and Holm-Hone (Section 4.5) equations as well as the BBM-BBM system (Section 4.6). For each dispersive wave model, conservative numerical methods are constructed and tested in some numerical experiments. Finally, we summarize the development and provide an outlook on future research in Section 5.

\section{Summation by parts operators}

In this section, periodic and non-periodic SBP operators are introduced at first in a general way. Afterwards, several examples of classical schemes are rephrased as SBP schemes. While there are generalizations of SBP methods $[26,27,81,87,88]$, we concentrate here on nodal collocation schemes where the boundary points are included. Thus, an interval $\left[x_{\min }, x_{\max }\right]$ is discretized using $\operatorname{a~grid~}^{1} \boldsymbol{x}=\left(\boldsymbol{x}_{1}, \ldots, \boldsymbol{x}_{N}\right)^{T}$, where $x_{\min }=\boldsymbol{x}_{1} \leq \boldsymbol{x}_{2} \leq \cdots \leq \boldsymbol{x}_{N}=x_{\max }$. A function $u:\left[x_{\min }, x_{\max }\right] \rightarrow \mathbb{R}$ is represented discretely on the grid $\boldsymbol{x}$ by its nodal values $\boldsymbol{u}=\left(\boldsymbol{u}_{1}, \ldots, \boldsymbol{u}_{N}\right)^{T}$, where $\boldsymbol{u}_{i}=u\left(\boldsymbol{x}_{i}\right)$. Multiplication of discrete grid functions $\boldsymbol{u}, \boldsymbol{v}$ is performed pointwise, i.e. $(\boldsymbol{u} \boldsymbol{v})_{i}=\boldsymbol{u}_{i} \boldsymbol{v}_{i}$.

\subsection{First-derivative operators}

The idea of summation by parts operators is to mimic integration by parts. Hence, compatible derivative and integration/quadrature operators are necessary [58, 94].

Definition 2.1. Given a grid $x$, a $p$-th order accurate $i$-th derivative matrix $D_{i}$ is a matrix that satisfies

$$
\forall k \in\{0, \ldots, p\}: \quad D_{i} x^{k}=k(k-1) \ldots(k-i+1) x^{k-i},
$$

with the convention $x^{0}=\mathbf{1}$ and $0 x^{k}=0$. We say $D_{i}$ is consistent if $p \geq 0$.

We will make frequent use of the vectors

$$
\boldsymbol{e}_{L}=(1,0, \ldots, 0)^{T}, \quad \boldsymbol{e}_{R}=(0, \ldots, 0,1)^{T},
$$

in order to evaluate grid functions at the left or right endpoint, respectively.

Definition 2.2. A first-derivative SBP operator consists of a grid $x$, a consistent first-derivative matrix $D_{1}$, and a symmetric and positive-definite matrix $M$, such that

$$
M D_{1}+D_{1}^{T} M=\boldsymbol{e}_{R} \boldsymbol{e}_{R}^{T}-\boldsymbol{e}_{L} \boldsymbol{e}_{L}^{T}
$$

We refer to $M$ as a mass matrix or norm matrix ${ }^{2}$.

First-derivative SBP operators mimic integration by parts via

$$
\begin{gathered}
\underbrace{\underbrace{\boldsymbol{u}^{T} M D_{1} \boldsymbol{v}+\boldsymbol{u}^{T} D_{1}^{T} M \boldsymbol{v}}}=\underbrace{\overbrace{\int_{\text {min }}^{x_{\max }} u\left(\partial_{x} v\right)+\int_{x_{\text {min }}}^{x_{\max }}\left(\partial_{x} u\right) v}^{\boldsymbol{u}^{T} \boldsymbol{e}_{R} \boldsymbol{e}_{R}^{T} \boldsymbol{v}-\boldsymbol{u}^{T} \boldsymbol{e}_{L} \boldsymbol{e}_{L}^{T} \boldsymbol{v},}}=\overbrace{u\left(x_{\max }\right) v\left(x_{\max }\right)-u\left(x_{\min }\right) v\left(x_{\min }\right)} .
\end{gathered}
$$

Of course, integration by parts requires some smoothness at the continuous level, e.g. absolute continuity of $u, v$. Such minimal smoothness assumptions are often used for formal a priori

\footnotetext{
${ }^{1}$ Here we include the possibility of repeated nodes in order to accommodate DG meshes in the most natural form.

${ }^{2}$ The term mass matrix is common for finite element methods. In the finite difference SBP community, the name norm matrix is more common.
} 
estimate. The purpose of SBP operators is to enable such a priori estimates also at the discrete level.

In the case of periodic boundary conditions (under which $x_{\min }$ and $x_{\max }$ are identical), the evaluations at the endpoints of the domain cancel. Hence, a periodic SBP operator can be defined as follows.

Definition 2.3. A periodic first-derivative SBP operator consists of a grid $\boldsymbol{x}$, a consistent first-derivative matrix $D_{1}$, and a symmetric and positive-definite matrix $M$ such that

$$
M D_{1}+D_{1}^{T} M=0
$$

We will often refer to an operator $D_{i}$ as a (periodic) SBP operator if the other operators (such as the mass matrix $M$ ) are clear from the context. We always assume derivative operators are consistent, but we will usually omit this term.

In periodic domains, first-derivative SBP operators are associated with skew-symmetric differentiation matrices. Hence, they are usually energy-conservative for linear hyperbolic problems. To allow for energy-dissipative SBP methods, upwind operators can be used, cf. [66, 70].

Definition 2.4. A first-derivative upwind SBP operator consists of a grid $\boldsymbol{x}$, consistent first-derivative matrices $D_{1, \pm}$, and a symmetric and positive-definite matrix $M$, such that

$$
M D_{1,+}+D_{1,-}^{T} M=\boldsymbol{e}_{R} \boldsymbol{e}_{R}^{T}-\boldsymbol{e}_{L} \boldsymbol{e}_{L}^{T}, \quad \frac{1}{2} M\left(D_{1,+}-D_{1,-}\right) \text { is negative semidefinite. }
$$

In matrix form, $D_{1,+}$ is biased toward the upper-triangular part (i.e. it has more nonzero entries in the upper part than in the lower) and $D_{1,-}$ is biased toward the lower-triangular part. The notion of upwind SBP operators can of course be extended to periodic domains.

Definition 2.5. A periodic first-derivative upwind SBP operator consists of a grid $\boldsymbol{x}$, consistent firstderivative matrices $D_{1, \pm}$, and a symmetric and positive-definite matrix $M$, such that

$$
M D_{1,+}+D_{1,-}^{T} M=0, \quad \frac{1}{2} M\left(D_{1,+}-D_{1,-}\right) \text { is negative semidefinite. }
$$

Remark 2.6. If $D_{1, \pm}$ are upwind SBP operators in a bounded or periodic domain, then $D_{1}=$ $\frac{1}{2}\left(D_{1,+}+D_{1,-}\right)$ is a (central) SBP operator. Furthermore, we can trivially obtain an upwind SBP operator from any (central) SBP operator $D_{1}$ by taking $D_{1,+}=D_{1}=D_{1,-}$. Though we term it upwind, this latter operator is of course non-dissipative. In general, upwind SBP operators introduce dissipation if $D_{1,+} \neq D_{1,-}$.

\subsection{Second-derivative operators}

Similarly to first-derivative SBP operators, second-derivative operators can be defined by mimicking integration by parts at the discrete level $[69,71]$.

Definition 2.7. A second-derivative SBP operator consists of a grid $\boldsymbol{x}$, a consistent second-derivative matrix $D_{2}$, a symmetric and positive-definite matrix $M$, and derivative vectors $\boldsymbol{d}_{L}, \boldsymbol{d}_{R}$ approximating the evaluation of the first derivative at the left/right endpoint as $\boldsymbol{d}_{L / R}^{T} \boldsymbol{u} \approx \boldsymbol{u}^{\prime}\left(x_{\min / \max }\right)$, such that

$$
M D_{2}=-A_{2}+\boldsymbol{e}_{R} \boldsymbol{d}_{R}^{T}-\boldsymbol{e}_{L} \boldsymbol{d}_{L}^{T}, \quad A_{2} \text { is symmetric and positive semidefinite. }
$$

First- and second-derivative SBP operators $D_{1}, D_{2}$ are said to be compatible if they are based on the same mass matrix $M$ and $-A_{2} \leq-D_{1}^{T} M D_{1}$ (in the sense of the induced quadratic forms). 
Remark 2.8. If SBP operators for different derivatives are applied in the same context, it will be assumed that they have the same mass matrix $M$.

Second-derivative SBP operators mimic integration by parts via

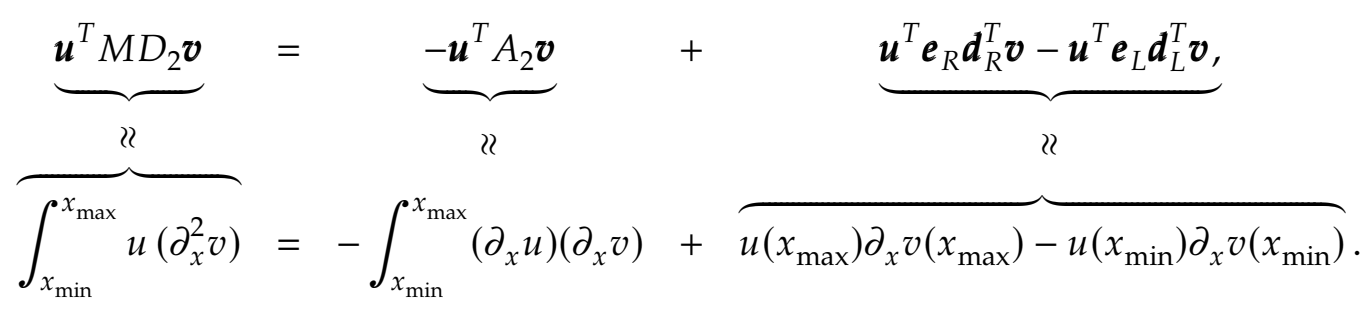

In periodic domains, the boundary terms vanish again, resulting in the following

Definition 2.9. A periodic second-derivative SBP operator consists of a grid $\boldsymbol{x}$, a consistent secondderivative matrix $D_{2}$, and a symmetric and positive-definite matrix $M$ such that

$$
M D_{2}=-A_{2}, \quad A_{2} \text { is symmetric and positive semidefinite. }
$$

One way to obtain a second-derivative SBP operator is to square a first-derivative operator: $D_{2}=D_{1}^{2}$. In the context of finite difference methods, the resulting operator is known as a widestencil operator. Compatibility of first- and second-derivative operators means that for any other choice of $D_{2}$ we have $-\boldsymbol{u}^{T} A_{2} \boldsymbol{u} \leq \boldsymbol{u}^{T} D_{1}^{T} M D_{1} \boldsymbol{u}$, i.e. the wide-stencil operator is the least dissipative (for the heat equation) of all compatible operators. In periodic domains with an even number of nodes, the highest frequency of grid oscillations is mapped to zero by such wide-stencil operators. To be able to damp such grid oscillations, it is preferable to use narrow-stencil operators [69]. For first-derivative upwind SBP operators $D_{1, \pm}$, both $D_{1,+} D_{1,-}$ and $D_{1,-} D_{1,+}$ are second-derivative SBP operators.

\subsection{Fourth-derivative operators}

Finally, fourth-derivative SBP operators can be defined as follows [65].

Definition 2.10. A fourth-derivative SBP operator consists of a grid $\boldsymbol{x}$, a consistent fourth-derivative matrix $D_{4}$, a symmetric and positive-definite matrix $M$, and derivative vectors $\boldsymbol{d}_{L, i}^{T}, \boldsymbol{d}_{R, i}^{T}$ approximating the evaluation of the $i$ th derivative at the left/right endpoint, such that

$$
M D_{2}=A_{4}+\boldsymbol{e}_{R} \boldsymbol{d}_{R, 3}^{T}-\boldsymbol{e}_{L} \boldsymbol{d}_{L, 3}^{T}-\boldsymbol{d}_{R, 1} \boldsymbol{d}_{R, 2}^{T}+\boldsymbol{d}_{L, 1} \boldsymbol{d}_{L, 2}^{T}, \quad A_{4} \text { is symmetric and positive semidefinite. }
$$

Fourth-derivative SBP operators mimic integration by parts via

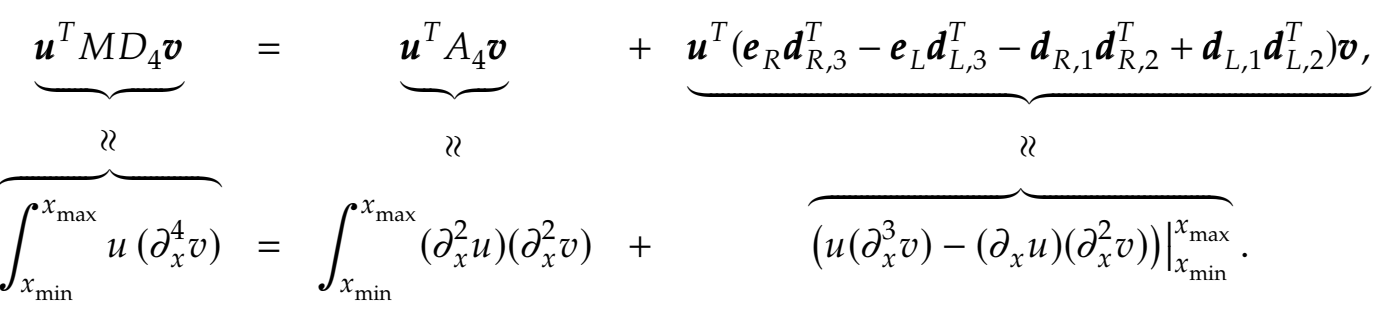

In periodic domains, the boundary terms vanish again, resulting in the following

Definition 2.11. A periodic fourth-derivative SBP operator consists of a grid $\boldsymbol{x}$, a consistent fourthderivative matrix $D_{4}$, and a symmetric and positive-definite matrix $M$ such that

$$
M D_{2}=A_{4}, \quad A_{4} \text { is symmetric and positive semidefinite. }
$$




\subsection{Finite difference and collocation methods}

Classical central finite difference methods result in periodic SBP operators with mass matrix

$$
M=\Delta x \mathrm{I},
$$

where $\Delta x=\left(x_{\max }-x_{\min }\right) / N$ is the grid spacing. Similarly, Fourier collocation methods [44, 57] and wavelet collocation methods [52] yield periodic SBP operators with the same mass matrix as central finite difference methods.

The first high-order finite difference SBP operators on bounded domains were proposed in [94] and later developed in several articles, e.g. [69]. Usually, these operators are associated with a uniform grid although some optimized versions employ adapted grid nodes near the boundaries [68].

Another class of SBP methods with diagonal mass matrix are spectral methods using nodal Lobatto-Legendre bases for polynomials of degree $p[22,45]$. These schemes result in SBP operators with a diagonal mass matrix $M=\operatorname{diag}\left(\omega_{0}, \ldots, \omega_{p}\right)$, where $\omega_{i}$ are the Lobatto-Legendre quadrature weights. The associated grid $\boldsymbol{x}$ is given by the Lobatto-Legendre quadrature nodes.

In periodic domains, finite difference schemes are usually applied globally. If multi-block finite difference or spectral collocation methods shall be used, they have to be constructed using (non-periodic) SBP operators on each element. Then, the elements have to be coupled either in a discontinuous way using SATs as described in Section 2.5 or continuously as described in Section 2.6.

\subsection{Nodal discontinuous Galerkin methods}

Multiple first-derivative SBP operators on bounded domains can be coupled in a discontinuous finite element way via SATs to construct global SBP operators, cf. [25, 27, 40]. The construction and the corresponding proof are reproduced here for completeness and convenience of the reader. Here and in the following, we consider only the coupling of two SBP operators on adjacent grids $\boldsymbol{x}_{l / r}$, where $\boldsymbol{x}_{l}=\left(\boldsymbol{x}_{i, l}\right)_{i=1}^{N_{l}}$ is the grid on the left- and $\boldsymbol{x}_{r}=\left(\boldsymbol{x}_{i, r}\right)_{i=1}^{N_{r}}$ is the grid on the right-hand side. Additionally, these grids have one node location in common: $\boldsymbol{x}_{N_{l}, l}=\boldsymbol{x}_{1, r}$.

Theorem 2.12. Consider two first-derivative SBP operators $D_{1, l / r}$ on the grids $\boldsymbol{x}_{l / r}$ with $x_{N_{l}, l}=x_{1, r}$. Then,

$$
D_{1}=\left(\begin{array}{cc}
D_{1, l}-\frac{1}{2} M_{l}^{-1} \boldsymbol{e}_{R, l} \boldsymbol{e}_{R, l}^{T} & \frac{1}{2} M_{l}^{-1} \boldsymbol{e}_{R, l} \boldsymbol{e}_{L, r}^{T} \\
-\frac{1}{2} M_{r}^{-1} \boldsymbol{e}_{L, r} \boldsymbol{e}_{R, l}^{T} & D_{1, r}+\frac{1}{2} M_{r}^{-1} \boldsymbol{e}_{L, r} \boldsymbol{e}_{L, r}^{T}
\end{array}\right), \quad M=\left(\begin{array}{cc}
M_{l} & 0 \\
0 & M_{r}
\end{array}\right),
$$

yields a first-derivative SBP operator on the joint grid $\boldsymbol{x}=\left(\boldsymbol{x}_{1, l}, \ldots, \boldsymbol{x}_{N_{l}, l}, \boldsymbol{x}_{1, r}, \ldots, \boldsymbol{x}_{N_{r}, r}\right)^{T}$ with $N=$ $N_{l}+N_{r}$ grid nodes. This SBP operator has the same order of accuracy as the less accurate operator of $D_{1, l / r}$.

Proof. The SBP property (2.3) is satisfied since

$$
\begin{aligned}
M D_{1}+D_{1}^{T} M & =\left(\begin{array}{cc}
M_{l} D_{1, l}+D_{1, l}^{T} M_{l}-\boldsymbol{e}_{R, l} \boldsymbol{e}_{R, l}^{T} & 0 \\
0 & M_{r} D_{1, r}+D_{1, r}^{T} M_{r}+\boldsymbol{e}_{L, r} \boldsymbol{e}_{L, r}^{T}
\end{array}\right) \\
& =\left(\begin{array}{cc}
-\boldsymbol{e}_{L, l} \boldsymbol{e}_{L, l}^{T} & 0 \\
0 & \boldsymbol{e}_{R, r} \boldsymbol{e}_{R, r}^{T}
\end{array}\right) .
\end{aligned}
$$

The order of accuracy can be checked by applying $D_{1}$ to a polynomial and noting that the interface terms vanish because of continuity of polynomials.

Remark 2.13. The derivative operator constructed in (2.15) yields

$$
M D_{1}\left(\begin{array}{l}
\boldsymbol{u}_{l} \\
\boldsymbol{u}_{r}
\end{array}\right)=\left(\begin{array}{c}
M_{l} D_{1, l} \boldsymbol{u}_{l}+\boldsymbol{e}_{R, l}\left(f^{\mathrm{num}}\left(\boldsymbol{e}_{R, l}^{T} \boldsymbol{u}_{l}, \boldsymbol{e}_{L, r}^{T} \boldsymbol{u}_{r}\right)-\boldsymbol{e}_{R, l}^{T} \boldsymbol{u}_{l}\right) \\
M_{r} D_{1, r} \boldsymbol{u}_{r}-\boldsymbol{e}_{L, r}\left(f^{\text {num }}\left(\boldsymbol{e}_{R, l}^{T} \boldsymbol{u}_{l}, \boldsymbol{e}_{L, r}^{T} \boldsymbol{u}_{r}\right)-\boldsymbol{e}_{L, r}^{T} \boldsymbol{u}_{r}\right)
\end{array}\right)
$$


where

$$
f^{\text {num }}\left(u_{-}, u_{+}\right)=\frac{u_{-}+u_{+}}{2}
$$

is the central numerical flux. This is the strong-form DG discretization on two elements using the central numerical flux between them and ignoring the other boundaries. For nodal DG methods on Lobatto-Legendre nodes, this strong form is equivalent to the prevalent weak form because of the SBP property (2.3), cf. [56], which discretizes (ignoring the boundary at $x_{1, l}$ )

$$
-\int_{x_{1, l}}^{x_{N_{l}, l}}\left(\partial_{x} \varphi_{l}\right) u+\varphi_{l}\left(x_{N_{l},}\right) f^{\text {num }}\left(u_{-}\left(x_{N_{l}, l}\right), u_{+}\left(x_{N_{r}, r}\right)\right)
$$

on the left element, where $\varphi_{l}$ is a test function.

Remark 2.14. The nodal DG methods used in this article are constructed by coupling multiple elements/blocks using the SBP operator with diagonal mass matrix determined by Lobatto-Legendre quadrature [55, Chapter 1] discontinuously as described in Theorem 2.12. The resulting discretization is the discontinuous Galerkin spectral element method [45]. For these methods, $\Delta x$ is the size (length) of one element.

Corollary 2.15. Coupling first-derivative SBP operators discontinuously as described in Theorem 2.12 on a periodic domain results in a periodic first-derivative SBP operator.

The discontinuously coupled first-derivative SBP operator $D_{1}$ in (2.15) can be squared to get a second-derivative SBP operator. This corresponds to the first method of Bassi and Rebay [10], cf. [9]. In order to increase the order of accuracy for DG methods for diffusive problems, the application of alternating upwind fluxes has been proposed in [29], resulting in the local DG (LDG) method [107], which is of the form $D_{2}=D_{1,+} D_{1,-}$ or $D_{2}=D_{1,-} D_{1,+}$ with first-derivative upwind SBP operators described in

Theorem 2.16. Consider two first-derivative upwind SBP operators $D_{1, \pm, l / r}$ on the grids $\boldsymbol{x}_{l / r}$ with $\boldsymbol{x}_{N_{l}, l}=$ $x_{1, r}$. Then,

$$
\begin{gathered}
D_{1,+}=\left(\begin{array}{cc}
D_{1,+, l}-M_{l}^{-1} \boldsymbol{e}_{R, l} \boldsymbol{e}_{R, l}^{T} & M_{l}^{-1} \boldsymbol{e}_{R, l} \boldsymbol{e}_{L, r}^{T} \\
0 & D_{1,+, r}
\end{array}\right), \quad D_{1,-}=\left(\begin{array}{cc}
D_{1,-, l} & 0 \\
-M_{r}^{-1} \boldsymbol{e}_{L, r} \boldsymbol{e}_{R, l}^{T} & D_{1,-, r}+M_{r}^{-1} \boldsymbol{e}_{L, r} \boldsymbol{e}_{L, r}^{T}
\end{array}\right), \\
M=\left(\begin{array}{cc}
M_{l} & 0 \\
0 & M_{r}
\end{array}\right)
\end{gathered}
$$

yield first-derivative upwind SBP operators on the joint grid $\boldsymbol{x}=\left(\boldsymbol{x}_{1, l}, \ldots, \boldsymbol{x}_{N_{l}, l}, \boldsymbol{x}_{1, r}, \ldots, \boldsymbol{x}_{N_{r}, r}\right)^{T}$ with $N=N_{l}+N_{r}$ nodes. These operators have the same order of accuracy as the less accurate of the given operators.

Proof. The upwind SBP property (2.6) can be verified by applying it for each operator $D_{1, \pm, l / r}$. Moreover,

$$
\begin{array}{r}
\left(\begin{array}{l}
\boldsymbol{u}_{l} \\
\boldsymbol{u}_{r}
\end{array}\right)^{T} M\left(D_{1,+}-D_{1,-}\right)\left(\begin{array}{l}
\boldsymbol{u}_{l} \\
\boldsymbol{u}_{r}
\end{array}\right)=\boldsymbol{u}_{l}^{T} M_{l}\left(D_{1,+, l}-D_{1,-, l}\right) \boldsymbol{u}_{l}+\boldsymbol{u}_{r}^{T} M_{r}\left(D_{1,+, r}-D_{1,-, r}\right) \boldsymbol{u}_{r} \\
-\left(\boldsymbol{e}_{R, l}^{T} \boldsymbol{u}_{l}\right)^{2}+2\left(\boldsymbol{e}_{R, l}^{T} \boldsymbol{u}_{l}\right)\left(\boldsymbol{e}_{L, r} \boldsymbol{u}_{r}\right)-\left(\boldsymbol{e}_{L, r} \boldsymbol{u}_{r}\right)^{2} \leq 0 .
\end{array}
$$

The order of accuracy can be checked as for Theorem 2.12 .

\subsection{Nodal continuous Galerkin methods}

An alternative to the discontinuous coupling of multiple elements is a continuous coupling of firstderivative operators as in continuous finite element methods, cf. [47, 48]. Continuous Galerkin methods have also been studied from the point of view of SBP operators in other articles, e.g. [1, 
$2,72]$. In contrast to the discontinuous coupling which uses the interface node twice and allows a multivalued solution there, the continuous coupling uses the interface node only once.

To describe the continuous coupling, indices of matrices will be denoted by subscripts using a syntax similar to MATLAB and Julia [12], i.e. $\left(M_{l}\right)_{1: N_{l}-1,1: N_{l}-1}$ denotes the upper left block of $M_{l}$ excluding the last column and row.

Theorem 2.17. Consider two first-derivative SBP operators $D_{1, l / r}$ on the grids $\boldsymbol{x}_{l / r}$ with $\boldsymbol{x}_{N_{l}, l}=\boldsymbol{x}_{1, r}$. Then,

$$
\begin{gathered}
D_{1}=M^{-1}\left(\begin{array}{ccc}
\left(M_{l} D_{1, l}\right)_{1: N_{l}-1,1: N_{l}-1} & \left(M_{l} D_{1, l}\right)_{1: N_{l}-1, N_{l}} & 0 \\
\left(M_{l} D_{1, l}\right)_{N_{l}, 1: N_{l}-1} & \left(M_{l} D_{1, l}\right)_{N_{l}, N_{l}}+\left(M_{r} D_{1, r}\right)_{1,1} & \left(M_{r} D_{1, r}\right)_{1,2: N_{r}} \\
0 & \left(M_{r} D_{1, r}\right)_{2: N_{r}, 1} & \left(M_{r} D_{1, r}\right)_{2: N_{r}, 2: N_{r}}
\end{array}\right), \\
M=\left(\begin{array}{ccc}
\left(M_{-}\right)_{1: N_{l}-1,1: N_{l}-1} & \left(M_{-}\right)_{1: N_{l}-1, N_{l}} & 0 \\
\left(M_{-}\right)_{N_{l}, 1: N_{l}-1} & \left(M_{-}\right)_{N_{l}, N_{l}}+M_{+, 1,1} & \left(M_{+}\right)_{1,2: N_{r}} \\
0 & \left(M_{+}\right)_{2: N_{r}, 1} & \left(M_{+}\right)_{2: N_{r}, 2: N_{r}}
\end{array}\right),
\end{gathered}
$$

yields a first-derivative SBP operator on the joint grid $\boldsymbol{x}=\left(\boldsymbol{x}_{1, l}, \ldots, \boldsymbol{x}_{N_{l}, l}=\boldsymbol{x}_{1, r}, \boldsymbol{x}_{2, r}, \ldots, \boldsymbol{x}_{N_{r}, r}\right)^{T}$ with $N=N_{l}+N_{r}-1$ grid nodes. This SBP operator has the same order of accuracy as the less accurate operator of $D_{1, l / r}$.

Proof. The new mass matrix $M$ is obviously symmetric and positive definite. Moreover,

$$
M D_{1}+D_{1}^{T} M=\left(\begin{array}{ccc}
\left(\boldsymbol{e}_{L, l} \boldsymbol{e}_{L, l}^{T}\right)_{1: N_{l}-1,1: N_{l}-1} & 0 & 0 \\
0 & 0 & 0 \\
0 & 0 & \left(\boldsymbol{e}_{R, r} \boldsymbol{e}_{R, r}^{T}\right)_{2: N_{r}, 2: N_{r}}
\end{array}\right)
$$

Again, the order of accuracy can be checked by applying $D_{1}$ to a polynomial.

Remark 2.18. The derivative constructed in (2.22) yields a strong-form CG discretization on two elements which (ignoring the other boundaries) is equivalent to the usual weak-form CG discretization

$$
-\int_{x_{1, l}}^{x_{N_{r}, r}}\left(\partial_{x} \varphi\right) u
$$

where $\varphi$ is a global test function, because of the SBP property (2.3).

Remark 2.19. The nodal CG methods used in this article are constructed by coupling multiple elements/blocks using the SBP operator with diagonal mass matrix determined by Lobatto-Legendre quadrature [55, Chapter 1] continuously as described in Theorem 2.17. For these methods, $\Delta x$ is the size (length) of one element.

Example 2.20. Coupling SBP operators using nodal Lobatto-Legendre bases for polynomials of degree $p=1$ continuously on a uniform mesh results in the classical finite difference SBP operator

$$
M=\Delta x\left(\begin{array}{ccccc}
1 / 2 & & & \\
& 1 & & \\
& & \ddots & \\
& & 1 & \\
& & & 1 / 2
\end{array}\right), \quad D_{1}=\frac{1}{\Delta x}\left(\begin{array}{ccccc}
-1 & 1 & & & \\
-1 / 2 & 0 & 1 / 2 & & \\
& \ddots & \ddots & \ddots & \\
& & -1 / 2 & 0 & 1 / 2 \\
& & & -1 & 1
\end{array}\right) .
$$

Corollary 2.21. Coupling first-derivative SBP operators continuously as described in Theorem 2.17 on a periodic domain results in a periodic first-derivative SBP operator.

Similarly to (central) SBP operators, first-derivative upwind SBP operators can also be coupled continuously. 
Theorem 2.22. Consider two first-derivative upwind SBP operators $D_{1, l / r, \pm}$ on the grids $\boldsymbol{x}_{l / r}$ with $\boldsymbol{x}_{N_{l, l}}=$ $x_{1, r}$. Then,

$$
\begin{gathered}
D_{1, \pm}=M^{-1}\left(\begin{array}{ccc}
\left(M_{l} D_{1, l, \pm}\right)_{1: N_{l}-1,1: N_{l}-1} & \left(M_{l} D_{1, l, \pm}\right)_{1: N_{l}-1, N_{l}} & 0 \\
\left(M_{l} D_{1, l, \pm}\right)_{N_{l}, 1: N_{l}-1} & \left(M_{l} D_{1, l, \pm}\right)_{N_{l}, N_{l}}+\left(M_{r} D_{1, r, \pm}\right)_{1,1} & \left(M_{r} D_{1, r, \pm}\right)_{1,2: N_{r}} \\
0 & \left(M_{r} D_{1, r, \pm}\right)_{2: N_{r}, 1} & \left(M_{r} D_{1, r, \pm}\right)_{2: N_{r}, 2: N_{r}}
\end{array}\right), \\
M=\left(\begin{array}{ccc}
M_{-, 1: N_{l}-1,1: N_{l}-1} & M_{-, 1: N_{l}-1, N_{l}} & 0 \\
M_{-, N_{l}, 1: N_{l}-1} & M_{-, N_{l}, N_{l}}+M_{+, 1,1} & M_{+, 1,2: N_{r}} \\
0 & M_{+, 2: N_{r}, 1} & M_{+, 2: N_{r}, 2: N_{r}}
\end{array}\right),
\end{gathered}
$$

yields first-derivative upwind SBP operators on the joint grid $\boldsymbol{x}=\left(\boldsymbol{x}_{1, l}, \ldots, \boldsymbol{x}_{N_{l}, l}=\boldsymbol{x}_{1, r}, \boldsymbol{x}_{2, r}, \ldots, \boldsymbol{x}_{N_{r}, r}\right)^{T}$ with $N=N_{l}+N_{r}-1$ nodes. These operators have the same order of accuracy as the less accurate given operators.

Proof. The mass matrix $M$ is the same as in Theorem 2.17 and hence symmetric and positive definite. Moreover,

$$
M D_{1,+}+D_{1,-}^{T} M=\left(\begin{array}{ccc}
\left(\boldsymbol{e}_{L, l} \boldsymbol{e}_{L, l}^{T}\right)_{1: N_{l}-1,1: N_{l}-1} & 0 & 0 \\
0 & 0 & 0 \\
0 & 0 & \left(\boldsymbol{e}_{R, r} \boldsymbol{e}_{R, r}^{T}\right)_{2: N_{r}, 2: N_{r}}
\end{array}\right)
$$

Furthermore, $M\left(D_{1,+}-D_{1,-}\right)$ is negative semidefinite.

Second-derivative operators can be coupled analogously.

Theorem 2.23. Consider two second-derivative SBP operators $D_{2, l / r}$ on the grids $\boldsymbol{x}_{l / r}$ with $\boldsymbol{x}_{N_{l}, l}=\boldsymbol{x}_{1, r}$. Then,

$$
\begin{gathered}
D_{2}=M^{-1}\left(\begin{array}{ccc}
\left(-A_{2, l}-\boldsymbol{e}_{L, l} \boldsymbol{d}_{L, l}^{T}\right)_{1: N_{l}-1,1: N_{l}-1} & \left(-A_{2, l}-\boldsymbol{e}_{L, l} \boldsymbol{d}_{L, l}^{T}\right)_{1: N_{l}-1, N_{l}} & 0 \\
\left(-A_{2, l}\right)_{N_{l}, 1: N_{l}-1} & \left(-A_{2, l}\right)_{N_{l}, N_{l}}+\left(-A_{2, r}\right)_{1,1} & \left(-A_{2, r}\right)_{1,2: N_{r}} \\
0 & \left(-A_{2, r}+\boldsymbol{e}_{R, r} \boldsymbol{d}_{R, r}^{T}\right)_{2: N_{r}, 1} & \left(-A_{2, r}+\boldsymbol{e}_{R, r} \boldsymbol{d}_{R, r}^{T}\right)_{2: N_{r}, 2: N_{r}}
\end{array}\right), \\
M=\left(\begin{array}{ccc}
M_{-, 1: N_{l}-1,1: N_{l}-1} & M_{-, 1: N_{l}-1, N_{l}} & 0 \\
M_{-, N_{l}, 1: N_{l}-1} & M_{-, N_{l}, N_{l}}+M_{+, 1,1} & M_{+, 1,2: N_{r}} \\
0 & M_{+, 2: N_{r}, 1} & M_{+, 2: N_{r}, 2: N_{r}}
\end{array}\right),
\end{gathered}
$$

yields a second-derivative SBP operator on the joint grid $\boldsymbol{x}=\left(\boldsymbol{x}_{1, l}, \ldots, \boldsymbol{x}_{N_{1}, l}=\boldsymbol{x}_{1, r}, \boldsymbol{x}_{2, r}, \ldots, \boldsymbol{x}_{N_{r}, r}\right)^{T}$ with $N=N_{l}+N_{r}-1$ grid nodes. This SBP operator has the same order of accuracy as the less accurate operator of $D_{2, l / r}$.

Proof. The new mass matrix $M$ is the same as in Theorem 2.17 and hence symmetric and positive definite. Additionally,

$$
\begin{aligned}
M D_{2}= & \left(\begin{array}{ccc}
\left(-A_{2, l}\right)_{1: N_{l}-1,1: N_{l}-1} & \left(-A_{2, l}\right)_{1: N_{l}-1, N_{l}} & 0 \\
\left(-A_{2, l}\right)_{N_{l}, 1: N_{l}-1} & \left(-A_{2, l}\right)_{N_{l}, N_{l}}+\left(-A_{2, r}\right)_{1,1} & \left(-A_{2, r}\right)_{1,2: N_{r}} \\
0 & \left(-A_{2, r}\right)_{2: N_{r}, 1} & \left(-A_{2, r}\right)_{2: N_{r}, 2: N_{r}}
\end{array}\right) \\
& +\left(\begin{array}{ccc}
\left(-\boldsymbol{e}_{L, l} \boldsymbol{d}_{L, l}^{T}\right)_{1: N_{l}-1,1: N_{l}-1} & \left(-\boldsymbol{e}_{L, l} \boldsymbol{d}_{L, l}^{T}\right)_{1: N_{l}-1, N_{l}} & 0 \\
0 & 0 & 0 \\
0 & \left(\boldsymbol{e}_{R, r} \boldsymbol{d}_{R, r}^{T}\right)_{2: N_{r}, 1} & \left(\boldsymbol{e}_{R, r} \boldsymbol{d}_{R, r}^{T}\right)_{2: N_{r}, 2: N_{r}}
\end{array}\right),
\end{aligned}
$$

where the first matrix is negative semidefinite. This is of the required form $M D_{2}=-A_{2}+\boldsymbol{e}_{R} \boldsymbol{d}_{R}^{T}-$ $\boldsymbol{e}_{L} \boldsymbol{d}_{L}^{T}$. Again, the order of accuracy can be checked by applying $D_{2}$ to a polynomial. 
Remark 2.24. Ignoring the outer boundaries, applying the SBP property (2.7) to the continuously coupled second-derivative operator yields a direct discretization of the weak form

$$
-\int_{x_{1, l}}^{x_{N_{r}, r}}\left(\partial_{x} \varphi\right)\left(\partial_{x} u\right) .
$$

Corollary 2.25. Coupling second-derivative SBP operators continuously as described in Theorem 2.23 on a periodic domain results in a periodic second-derivative SBP operator.

Example 2.26. Coupling second-derivative SBP operators using nodal Lobatto-Legendre bases for polynomials of degree $p=1$ continuously on a uniform mesh results in

$$
M=\Delta x\left(\begin{array}{cccc}
1 / 2 & & & \\
& 1 & & \\
& & \ddots & \\
& & 1 & \\
& & & 1 / 2
\end{array}\right), \quad D_{2}=\frac{1}{\Delta x^{2}}\left(\begin{array}{ccccc}
0 & 0 & & & \\
1 & -2 & 1 & & \\
& \ddots & \ddots & \ddots & \ddots \\
& & 1 & -2 & 1 \\
& & & 0 & 0
\end{array}\right),
$$

which is very similar to the narrow-stencil second-derivative SBP operator

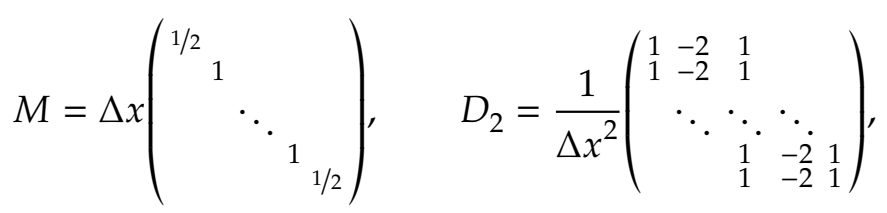

of [69] but uses a different boundary closure.

\subsection{Some useful properties of periodic SBP operators}

Here, we gather some properties of periodic SBP operators that will be useful to prove conservation properties later in the article.

Lemma 2.27. Periodic first, second, and fourth-derivative operators satisfy $\mathbf{1}^{T} M D_{i}=\mathbf{0}^{T}$. Periodic firstderivative upwind SBP operators satisfy $\mathbf{1}^{T} M D_{1, \pm}=\mathbf{0}^{T}$.

Proof. Applying the defining conditions (2.5) \& (2.10) \& (2.13) and consistency of the derivative operators yields

$$
\begin{aligned}
& \mathbf{1}^{T} M D_{1}=-\mathbf{1}^{T} D_{1}^{T} M=\mathbf{0}^{T}, \\
& 1^{T} M D_{2}=\mathbf{1}^{T} D_{2}^{T} M=\mathbf{0}^{T}, \\
& 1^{T} M D_{4}=\mathbf{1}^{T} D_{4}^{T} M=0^{T} .
\end{aligned}
$$

Similarly,

$$
\mathbf{1}^{T} M D_{1, \pm}=-\mathbf{1}^{T} D_{1, \mp}^{T} M=\mathbf{0}^{T}
$$

Lemma 2.28. If $D_{2}$ is a periodic second-derivative SBP operator with mass matrix $M$, then $\mathbf{1}^{T} M\left(\mathrm{I}-D_{2}\right)^{-1}=$ $\mathbf{1}^{T} M$.

Proof. Since $M\left(\mathrm{I}-\mathrm{D}_{2}\right)^{-1}$ is symmetric,

$$
\mathbf{1}^{T} M\left(\mathrm{I}-D_{2}\right)^{-1}=\left(M\left(\mathrm{I}-D_{2}\right)^{-1} \mathbf{1}\right)^{T}=(M \mathbf{1})^{T} .
$$

Here, we used $\left(\mathrm{I}-D_{2}\right) \mathbf{1}=1$, since $D_{2} 1=\mathbf{0}$ for any consistent second-derivative approximation $D_{2}$. 
Lemma 2.29. If $D_{1}, D_{2}$ are commuting periodic first- and second-derivative SBP operators with the same mass matrix $M$, then $M\left(\mathrm{I}-D_{2}\right)^{-1} D_{1}$ is skew-symmetric.

Proof. Since

$$
\mathrm{I}-D_{2}=M^{-1}\left(\mathrm{I}-M D_{2} M^{-1}\right) M=M^{-1}\left(\mathrm{I}-D_{2}^{T}\right) M
$$

we have

$$
M\left(\mathrm{I}-D_{2}\right)^{-1} D_{1}=\left(\mathrm{I}-D_{2}^{T}\right)^{-1} M D_{1}=-\left(\mathrm{I}-D_{2}^{T}\right)^{-1} D_{1}^{T} M=-D_{1}^{T}\left(\mathrm{I}-D_{2}^{T}\right)^{-1} M .
$$

In order to use the lemmas above, we will need pairs of first- and second-derivative operators that commute. In the following examples we see that certain natural approaches lead to commuting operators, while others do not.

Example 2.30. The finite difference methods described in Section 2.4 in periodic domains result in commuting first- and second-derivative operators, since these can be represented by circulant matrices, i.e. by Toeplitz matrices where each row is obtained from the preceding row by cyclically shifting every entry one step to the right [61, Section C.7].

Example 2.31. Let $D_{1}$ be a first-derivative SBP operator and let $D_{1, \pm} \neq D_{1}$ be first-derivative upwind SBP operators all with the same mass matrix $M$. Clearly, the first- and second-derivative operators $\left(D_{1}, D_{1}^{2}\right)$ commute. On the other hand, $D_{1}$ does not in general commute with the second-derivative operators $D_{2}=D_{1,-} D_{1,+}$ or $D_{2}=D_{1,+} D_{1,-}$. Furthermore, in general $M D_{2} D_{1}$ is not skew-symmetric.

For example, in a periodic domain $[-1,3]$ with two elements using nodal Lobatto-Legendre bases for polynomials of degree $p=1$, we have

$$
\begin{gathered}
D_{1}=\left(\begin{array}{cccc}
0 & \frac{1}{2} & 0 & -\frac{1}{2} \\
-\frac{1}{2} & 0 & \frac{1}{2} & 0 \\
0 & -\frac{1}{2} & 0 & \frac{1}{2} \\
\frac{1}{2} & 0 & -\frac{1}{2} & 0
\end{array}\right), \quad D_{1,-}=\left(\begin{array}{cccc}
\frac{1}{2} & \frac{1}{2} & 0 & -1 \\
-\frac{1}{2} & \frac{1}{2} & 0 & 0 \\
0 & -1 & \frac{1}{2} & \frac{1}{2} \\
0 & 0 & -\frac{1}{2} & \frac{1}{2}
\end{array}\right), \quad D_{1,+}=\left(\begin{array}{cccc}
-\frac{1}{2} & \frac{1}{2} & 0 & 0 \\
-\frac{1}{2} & -\frac{1}{2} & 1 & 0 \\
0 & 0 & -\frac{1}{2} & \frac{1}{2} \\
1 & 0 & -\frac{1}{2} & -\frac{1}{2}
\end{array}\right), \\
M D_{1,-} D_{1,+} D_{1}=\left(\begin{array}{cccc}
\frac{1}{4} & -\frac{5}{4} & -\frac{1}{4} & \frac{5}{4} \\
\frac{1}{4} & -\frac{1}{4} & -\frac{1}{4} & \frac{1}{4} \\
-\frac{1}{4} & \frac{5}{4} & \frac{1}{4} & -\frac{5}{4} \\
-\frac{1}{4} & \frac{1}{4} & \frac{1}{4} & -\frac{1}{4}
\end{array}\right), \quad M D_{1,+} D_{1,-} D_{1}=\left(\begin{array}{cccc}
\frac{1}{4} & -\frac{1}{4} & -\frac{1}{4} & \frac{1}{4} \\
\frac{5}{4} & -\frac{1}{4} & -\frac{5}{4} & \frac{1}{4} \\
-\frac{1}{4} & \frac{1}{4} & \frac{1}{4} & -\frac{1}{4} \\
-\frac{5}{4} & \frac{1}{4} & \frac{5}{4} & -\frac{1}{4}
\end{array}\right) .
\end{gathered}
$$

Hence, the second-derivative operator obtained via the LDG procedure does not, in general, commute with the corresponding first-derivative operator. Using instead the first method of Bassi and Rebay, $D_{2}=D_{1}^{2}$, the operators commute.

Example 2.32. Similarly to the discontinuous coupling described in Example 2.31, a continuous coupling also does not result in commuting first- and second-derivative operators and $M D_{1} D_{2}$ is not skew-symmetric in general. In a periodic domain $[-1,3]$ with two elements using nodal Lobatto-Legendre bases for polynomials of degree $p=2$,

$$
\begin{aligned}
D_{1} & =\left(\begin{array}{cccc}
0 & 1 & 0 & -1 \\
-\frac{1}{2} & 0 & \frac{1}{2} & 0 \\
0 & -1 & 0 & 1 \\
\frac{1}{2} & 0 & -\frac{1}{2} & 0
\end{array}\right), & D_{2}=\left(\begin{array}{cccc}
-\frac{7}{2} & 2 & -\frac{1}{2} & 2 \\
1 & -2 & 1 & 0 \\
-\frac{1}{2} & 2 & -\frac{7}{2} & 2 \\
1 & 0 & 1 & -2
\end{array}\right), \\
M= & \left.\begin{array}{cccc}
\frac{2}{3} & 0 & 0 & 0 \\
0 & \frac{4}{3} & 0 & 0 \\
0 & 0 & \frac{2}{3} & 0 \\
0 & 0 & 0 & \frac{4}{3}
\end{array}\right), & M D_{2} D_{1}=\left(\begin{array}{cccc}
0 & -2 & 0 & 2 \\
\frac{4}{3} & 0 & -\frac{4}{3} & 0 \\
0 & 2 & 0 & -2 \\
-\frac{4}{3} & 0 & \frac{4}{3} & 0
\end{array}\right) .
\end{aligned}
$$

Hence, the second-derivative operator obtained via the continuous coupling procedure does not, in general, commute with the corresponding first-derivative operator. Moreover, $M D_{2} D_{1}$ is indefinite. In order to obtain commuting operators, one may again to use the squared first-derivative operator as a second-derivative operator, resulting in a wide-stencil operator. 
Lemma 2.33. If $D_{1, \pm}$ are periodic upwind SBP operators, $M\left(\mathrm{I}-D_{1,-} D_{1,+}\right)^{-1} D_{1,-}$ is positive semidefinite and $M\left(\mathrm{I}-D_{1,+} D_{1,-}\right)^{-1} D_{1,+}$ is negative semidefinite.

Proof. It suffices to check whether

$$
\left\langle\boldsymbol{u},\left(\mathrm{I}-D_{1,-} D_{1,+}\right)^{-1} D_{1,-} \boldsymbol{u}\right\rangle_{M} \geq 0
$$

for all $\boldsymbol{u}$. Equivalently, one can consider $\boldsymbol{w}=\left(\mathrm{I}-D_{1,-} D_{1,+}\right)^{-1} \boldsymbol{u}$ and compute

$$
\begin{aligned}
& \left\langle\boldsymbol{u},\left(\mathrm{I}-D_{1,-} D_{1,+}\right)^{-1} D_{1,-} \boldsymbol{u}\right\rangle_{M} \\
= & \left\langle\boldsymbol{w}, D_{1,-}\left(\mathrm{I}-D_{1,-} D_{1,+}\right) \boldsymbol{w}\right\rangle_{M}=-\left\langle\boldsymbol{w},\left(\mathrm{I}-D_{1,-} D_{1,+}\right) D_{1,+} \boldsymbol{w}\right\rangle_{M} \\
= & \frac{1}{2}\left\langle\boldsymbol{w},\left(D_{1,-}-D_{1,+}\right) \boldsymbol{w}\right\rangle_{M}-\frac{1}{2}\left\langle\boldsymbol{w}, D_{1,-}^{2} D_{1,+} \boldsymbol{w}\right\rangle_{M}+\frac{1}{2}\left\langle\boldsymbol{w}, D_{1,-} D_{1,+}^{2} \boldsymbol{w}\right\rangle_{M} \\
= & \frac{1}{2}\left\langle\boldsymbol{w},\left(D_{1,-}-D_{1,+}\right) \boldsymbol{w}\right\rangle_{M}+\frac{1}{2}\left\langle D_{1,+} \boldsymbol{w},\left(D_{1,-}-D_{1,+}\right) D_{1,+} \boldsymbol{w}\right\rangle_{M} \geq 0 .
\end{aligned}
$$

The other assertion is verified by exchanging + and - .

\subsection{Choice of appropriate split forms}

Since SBP operators mimic integration by parts discretely, proofs of invariant conservation can be transferred to the discrete level directly if this tool is used. However, systematic integration by parts is often coupled with the application of the product or chain rule, which do not, in general, hold discretely [82]. Instead, split forms can be used in a systematic way. A general recipe for constructing discretely conservative split forms is given in the following.

Check whether conservation of a nonlinear invariant can be proved using only integration by parts and symmetry properties of differential operators. If so, apply the same steps discretely using SBP operators. Otherwise, write nonlinear terms as linear combinations of different split forms obtained by the product/chain rule and repeat the procedure.

As an example, consider Burgers' equation

$$
\partial_{t} u(t, x)+\partial_{x} \frac{u(t, x)^{2}}{2}=0
$$

in a periodic domain. Conservation of the $L^{2}$ norm can be shown by applying the chain rule as in

$$
\frac{\mathrm{d}}{\mathrm{d} t} \frac{1}{2}\|u(t)\|_{L^{2}}^{2}=\int u \partial_{t} u=-\frac{1}{2} \int u \partial_{x} u^{2}=-\frac{1}{3} \int \partial_{x} u^{3}=0 .
$$

In order to achieve discrete conservation, we look for a way to show conservation using only integration by parts. To this end, consider the general splitting of the nonlinear term:

$$
\partial_{x} \frac{u^{2}}{2}=\alpha \partial_{x} u^{2}+(1-2 \alpha) u \partial_{x} u
$$

where $\alpha \in \mathbb{R}$ is a real parameter. The energy method using only integration by parts yields

$$
\frac{\mathrm{d}}{\mathrm{d} t} \frac{1}{2}\|u(t)\|_{L^{2}}^{2}=\int u \partial_{t} u=-\alpha \int u \partial_{x} u^{2}-(1-2 \alpha) \int u^{2} \partial_{x} u=(1-3 \alpha) \int u \partial_{x} u^{2} .
$$

Energy conservation can be obtained by taking $\alpha=1 / 3$, and this leads naturally to a conservative numerical method. 
The quadratic nonlinearity of Burgers' equation appears in several of the dispersive wave equations considered in this article, and conservative methods for them can be designed using the split form just derived. Such split forms can also be generalized to higher-order polynomial nonlinearities [80, Section 4.5] and there are efficient means to evaluate these for FD, DG, and CG methods $[46,79]$. The same idea can even be applied to get conservative discretizations for non-polynomial nonlinearities [43], but the resulting schemes cannot be interpreted in terms of split forms.

\section{Relaxation methods in time}

In the previous section, we have developed tools for producing conservative spatial discretizations; in the following sections, these will be applied to obtain conservative semi-discrete methods for specific wave equations. These methods reduce an initial boundary value PDE to an initial value ODE system

$$
u^{\prime}(t)=f(u(t)), \quad u(0)=u_{0},
$$

satisfying a conservation property

$$
\frac{\mathrm{d}}{\mathrm{d} t} J(u)=0
$$

for some nonlinear invariant $J$. Herein we employ one-step integration methods and we enforce the conservation property discretely in time, so that $J\left(u^{n}\right)=J\left(u^{n-1}\right)=J\left(u_{0}\right)$. We can achieve this by combining our conservative spatial discretizations with relaxation methods in time $[53,84,85$, 89].

We start with a Runge-Kutta method

$$
\begin{aligned}
y_{i} & =u^{n}+\Delta t \sum_{j=1}^{s} a_{i j} f\left(t_{n}+c_{j} \Delta t, y_{j}\right), \quad i \in\{1, \ldots, s\}, \\
u\left(t_{n}+\Delta t\right) \approx u^{n+1} & =u^{n}+\Delta t \sum_{i=1}^{s} b_{i} f\left(t_{n}+c_{i} \Delta t, y_{i}\right),
\end{aligned}
$$

and define

$$
d^{n}:=\sum_{i=1}^{s} b_{i} f_{i},
$$

where we use the shorthand $f_{i}:=f\left(t_{n}+c_{i} \Delta t, y_{i}\right)$. In general the new solution $u^{n}$ will not be conservative, so we replace the update formula (3.3b) with an update in the same direction but of a (possibly) different length:

$$
u\left(t_{n}+\gamma^{n} \Delta t\right) \approx u_{\gamma}^{n+1}=u^{n}+\gamma^{n} \Delta t d^{n} .
$$

The relaxation parameter $\gamma^{n}$ is chosen as a solution of the conservation equation

$$
J\left(u_{\gamma}^{n+1}\right)=J\left(u^{n}\right)
$$

Thus $\gamma^{n}$ is obtained by solving a scalar nonlinear equation, using some root-finding method. By the general theory on relaxation methods, there is exactly one root $\gamma^{n}=1+O\left(\Delta t^{p-1}\right)$ of (3.6) [85, Theorem 2.14]. Other possible roots, such as the trivial solution $\gamma=0$, are further away from unity. For quadratic invariants $J$, these two roots are the only roots and can be computed explicitly. Similarly, the root $\gamma^{n}$ closest to unity can also be computed explicitly for cubic invariants such as for the BBM-BBM system discussed in Section 4.6. However, these explicit formulas can be sensitive 
to floating point errors; we have found that the application of standard root finding algorithms such as those of [3] is efficient and often results in more accurate solutions.

The resulting solution $u_{\gamma}^{n+1}$ conserves the invariant $J$ by construction. In contrast to projection methods, the relaxation approach also automatically conserves linear invariants (as long as the semi-discretization conserves them). The solution (3.5) has the same local order of accuracy as that given by the original Runge-Kutta method (3.3).

The use of relaxation Runge-Kutta methods in this context may be viewed as an application of the ideas developed in [84]. For some dispersive wave problems, the value of $J$ might change over time due to boundary conditions or the presence of dissipative terms; in this case relaxation methods can also be used to improve the accuracy of the time evolution of $J[53,89]$. For more details regarding the properties of relaxation methods, including multistep relaxation methods, we refer the reader to [85].

\section{Conservative discretizations of specific wave equations}

In this section we develop new conservative discretizations for several nonlinear dispersive wave equations, using the tools from the previous two sections. Most of the discretizations are based on using appropriate splittings for nonlinear terms and using a basis with a diagonal mass matrix. For some of the equations studied (namely, the Fornberg-Whitham, Camassa-Holm, Hone-Holm, and BBM-BBM equations) we also require that the various discrete derivative operators commute. For other equations (namely, the BBM and Degasperis-Procesi equations) this is not necessary.

Most of the proposed methods (all except for the BBM-BBM system) require that the discrete derivative operators have a diagonal mass matrix. Alternatively, one can achieve conservation by discretizing the conservative form of the equation and using exact integration of all variational forms. The latter approach has been used to construct conservative methods, e.g. in $[62,105,108$, 111]. An advantage of the present approach is that exact integration of the nonlinear terms is not necessary if the mass matrix is diagonal.

We focus on the development of the discretizations and proofs of their conservation properties, but we also provide results of simple numerical tests that confirm the theoretical properties of the schemes. Numerical results for each equation are described in the corresponding section and are also summarized at the end of this work in Table 1. More extensive numerical experiments, such as studies of solitary wave interaction, are left to future work.

We test the accuracy using the method of manufactured solutions, which consists of choosing a smooth solution a priori and then adding a source term $f(t, x)$ to the PDE so that the solution satisfies it [91]. In order to isolate the spatial discretization errors, we discretize in time using the fifth-order explicit Runge-Kutta pair of [97] with adaptive time stepping and a local error tolerance of $1.0 \times 10^{-12}$, without relaxation. For all of the spatial discretizations proposed, linearized stability analysis suggests that the maximum stable time step is either proportional to $\Delta x$ or independent of $\Delta x$, so explicit time integration can be efficient.

Conservation tests are performed using solitary wave solutions, obtained either analytically or via the Petviashvili method [77] using a Fourier collocation method with $N=2^{16}$ nodes. For these tests we use the classical 4th-order method of Kutta [59], with relaxation. Other space and time discretizations have been tested as well but are not shown here. We remark in advance that in all cases, these tests demonstrate conservation of all linear invariants and the selected nonlinear invariant. Results for specific discretizations are shown only when they reveal something of further interest.

In the following, all errors of the form $\left\|u-u_{\text {ana }}\right\|$, where $u$ is the numerical approximation and $u_{\text {ana }}$ the analytical solution, are computed using the discrete norm induced by the mass matrix $M$. These discrete $L^{2}$ errors are used to compute the experimental order of convergence (EOC). 


\subsection{Benjamin-Bona-Mahony equation}

Consider the Benjamin-Bona-Mahony equation [11] (also known as regularized long wave equation)

$$
\begin{aligned}
\left(\mathrm{I}-\partial_{x}^{2}\right) \partial_{t} u(t, x)+\partial_{x} f(u(t, x))+\partial_{x} u(t, x) & =0, \quad t \in(0, T), x \in\left(x_{\min }, x_{\max }\right), \\
u(0, x) & =u^{0}(x), \quad x \in\left[x_{\min }, x_{\max }\right], \\
f(u) & =\frac{u^{2}}{2},
\end{aligned}
$$

with periodic boundary conditions, which can also be written as

$$
\partial_{t} u(t, x)+\left(\mathrm{I}-\partial_{x, P}^{2}\right)^{-1} \partial_{x}(f(u(t, x))+u(t, x))=0,
$$

where $\left(\mathrm{I}-\partial_{x, P}^{2}\right)^{-1}$ is the inverse of the elliptic operator $\mathrm{I}-\partial_{x}^{2}$ with periodic boundary conditions. The functionals

$$
\begin{aligned}
& J_{1}^{\mathrm{BBM}}(u)=\int_{x_{\text {min }}}^{x_{\max }} u, \\
& J_{2}^{\mathrm{BBM}}(u)=\frac{1}{2} \int_{x_{\min }}^{x_{\max }}\left(u^{2}+\left(\partial_{x} u\right)^{2}\right)=\frac{1}{2} \int_{x_{\min }}^{x_{\max }} u\left(\mathrm{I}-\partial_{x}^{2}\right) u, \\
& J_{3}^{\mathrm{BBM}}(u)=\int_{x_{\text {min }}}^{x_{\text {max }}}(u+1)^{3},
\end{aligned}
$$

are invariants of solutions [75]. In the following, we will construct numerical methods that conserve the linear (4.3a) and quadratic (4.3b) invariants but not necessarily the cubic invariant (4.3c).

\subsubsection{Conservative numerical methods}

The rate of change of the quadratic invariant $(4.3 \mathrm{~b})$ is

$$
\int_{x_{\min }}^{x_{\max }} u\left(\mathrm{I}-\partial_{x}^{2}\right) \partial_{t} u=-\int_{x_{\min }}^{x_{\max }} u \partial_{x} \frac{u^{2}}{2}-\int_{x_{\min }}^{x_{\max }} u \partial_{x} u .
$$

The first integral on the right-hand side is exactly the one appearing in the energy rate for Burgers' equation (2.43). Hence, the linear and quadratic invariants are conserved semidiscretely if periodic SBP operators are employed and the semidiscretization uses the split form

$$
\partial_{t} \boldsymbol{u}+\left(\mathrm{I}-D_{2}\right)^{-1}\left(\frac{1}{3} D_{1} \boldsymbol{u}^{2}+\frac{1}{3} \boldsymbol{u} D_{1} \boldsymbol{u}+D_{1} \boldsymbol{u}\right)=\mathbf{0} .
$$

Theorem 4.1. If $D_{1}$ is a periodic first-derivative SBP operator with diagonal mass matrix $M$ and $D_{2}$ is a periodic second-derivative SBP operator, then the semidiscretization (4.5) conserves the invariants (4.3a) and (4.3b) of (4.1).

Proof. The linear invariant (4.3a) is conserved since

$$
\begin{aligned}
\frac{\mathrm{d}}{\mathrm{d} t} \mathbf{1}^{T} M \boldsymbol{u} & =\mathbf{1}^{T} M \partial_{t} \boldsymbol{u}=-\mathbf{1}^{T} M\left(\mathrm{I}-D_{2}\right)^{-1}\left(\frac{1}{3} D_{1} \boldsymbol{u}^{2}+\frac{1}{3} \boldsymbol{u} D_{1} \boldsymbol{u}+D_{1} \boldsymbol{u}\right) \\
& =-\mathbf{1}^{T} M\left(\frac{1}{3} D_{1} \boldsymbol{u}^{2}+\frac{1}{3} \boldsymbol{u} D_{1} \boldsymbol{u}+D_{1} \boldsymbol{u}\right)=0,
\end{aligned}
$$

where Lemma 2.28 has been used.

Since $\mathrm{I}-D_{2}$ is a symmetric operator, the semidiscrete rate of change of the quadratic invariant $(4.3 b)$ is

$$
\frac{1}{2} \frac{\mathrm{d}}{\mathrm{d} t} \boldsymbol{u}^{T} M\left(\mathrm{I}-D_{2}\right) \boldsymbol{u}=\boldsymbol{u}^{T} M\left(\mathrm{I}-D_{2}\right) \partial_{t} \boldsymbol{u}=-\frac{1}{3} \boldsymbol{u}^{T} M D_{1} \boldsymbol{u}^{2}-\frac{1}{3} \boldsymbol{u}^{T} M \boldsymbol{u} D_{1} \boldsymbol{u}-\boldsymbol{u}^{T} M D_{1} \boldsymbol{u}=0 .
$$


Remark 4.2. Conservative linearly- and nonlinearly-implicit second-order finite difference schemes for the BBM equation based on the discrete variational derivative method were proposed in [54]. $\triangleleft$

Remark 4.3. Starting from the conservative semidiscretization (4.5), energy-dissipative semidiscretizations can be constructed as well. For example, the linear term $\left(I-D_{2}\right)^{-1} D_{1} \boldsymbol{u}$ can be replaced by $\left(\mathrm{I}-D_{1,-} D_{1,+}\right)^{-1} D_{1,-}$. Then, the linear invariant $J_{1}^{\mathrm{BBM}}$ is still conserved and the contribution to the energy rate becomes $-\boldsymbol{u}^{T} M\left(\mathrm{I}-D_{1,-} D_{1,+}\right)^{-1} D_{1,-} \boldsymbol{u} \leq 0$ because of Lemma 2.33. Additional dissipation can be introduced in DG schemes by applying a dissipative numerical flux such as Godunov's flux at interfaces for the nonlinear term. Another possibility is to add artificial dissipation proportional to $D_{1,-}-D_{1,+}$ to the nonlinear term on left hand side of (4.5).

\subsubsection{Convergence study in space}

To study convergence, the method of manufactured solutions [91] is applied to

$$
u(t, x)=\mathrm{e}^{t / 2} \sin (2 \pi(x-t / 2)), \quad(x, t) \in[0,1] \times[0,1],
$$

with periodic boundary conditions. Results for the semidiscretization (4.5) are shown in Figure 1.

For central finite difference methods with order of accuracy $p$, we observe an order of convergence approximately equal to $p$. The results for wide-stencil and narrow-stencil second-derivative operators are similar but the narrow-stencil operators result in errors that are smaller by up to an order of magnitude.

For nodal continuous Galerkin methods using Lobatto-Legendre bases, the results depend on the choice of the second-derivative operator. Wide-stencil operators $D_{2}=D_{1}^{2}$ with polynomial degree $p$ yield $\mathrm{EOC} \approx p+1$ for $p$ odd and $\mathrm{EOC} \approx p$ for $p$ even. In contrast, the usual narrow-stencil approximation (Theorem 2.23) results in an $\mathrm{EOC} \approx p+1$ for $p=1$ and an $\mathrm{EOC} \approx p+2$ for $p>1$.

A similar observation can be made for nodal discontinuous Galerkin methods. There, widestencil operators $D_{2}=D_{1}^{2}$ yield EOC $\approx p+1$ for even polynomial degrees $p$ and EOC $\approx p$ for odd $p$. The narrow-stencil LDG operator $D_{2}=D_{1,+} D_{1,-}$ results in an EOC $\approx p+1$ for all $p$.

\subsubsection{Conservation of invariants}

To test the conservation properties of the scheme, we use the traveling wave solution

$$
u(t, x)=A \cosh (K(x-c t)), \quad A=3(c-1), \quad K=\frac{1}{2} \sqrt{1-1 / c},
$$

with speed $c=1.2$ in the periodic domain $[-90,90]$. The classical fourth-order Runge-Kutta method RK4 [59] is used with relaxation (as described in Section 3) to enforce conservation of $J_{2}^{\mathrm{BBM}}$. Results for all spatial discretizations show conservation of the linear and quadratic invariants, to within roundoff error. Interestingly, applying relaxation to conserve the quadratic invariant (4.3b) improves the evolution of the cubic invariant (4.3c) as well. An example showing this behavior, with a Fourier pseudospectral method in space, can be seen in Figure 2. Results for other spatial discretizations are similar.

Moreover, the deviation of the cubic invariant seems to be bounded and approximately constant. This indicates that the remaining error of the energy-conservative relaxation method is mainly a phase error and not an amplitude/shape error.

\subsection{Fornberg-Whitham equation}

Consider the Fornberg-Whitham equation [101]

$$
\begin{aligned}
& \left(\mathrm{I}-\partial_{x}^{2}\right) \partial_{t} u(t, x)+\left(\mathrm{I}-\partial_{x}^{2}\right) \partial_{x} f(u(t, x))+\partial_{x} u(t, x)=0, \quad t \in(0, T), x \in\left(x_{\min }, x_{\max }\right), \\
& u(0, x)=u^{0}(x), \quad x \in\left[x_{\min }, x_{\max }\right], \\
& f(u)=\frac{u^{2}}{2}
\end{aligned}
$$




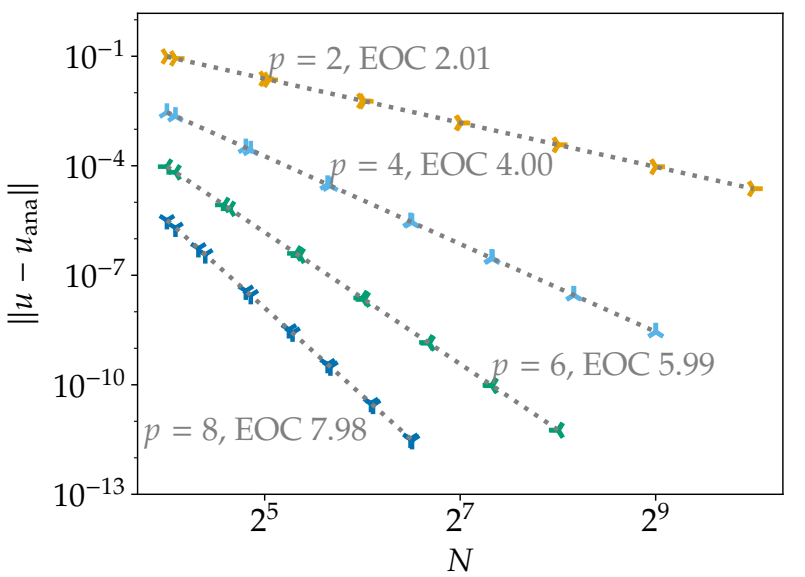

(a) Finite difference methods, $D_{2}=D_{1}^{2}$.

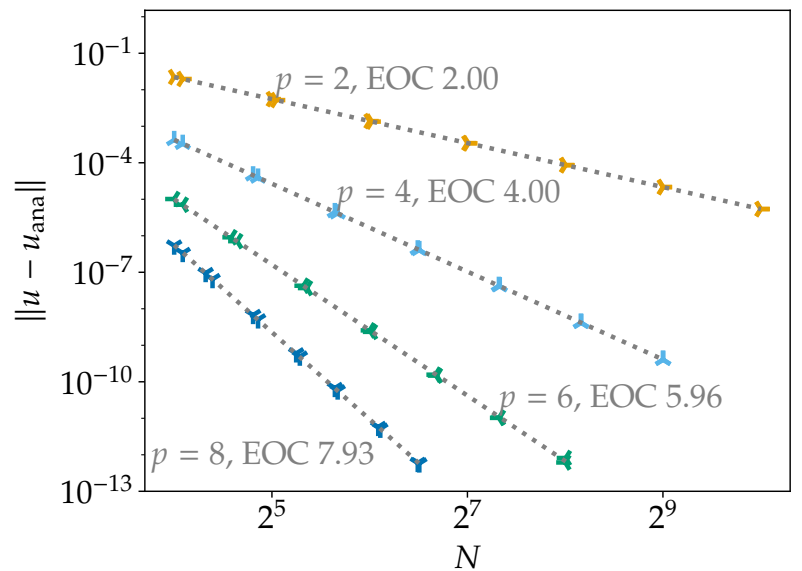

(b) FD methods with narrow-stencil $D_{2}$.

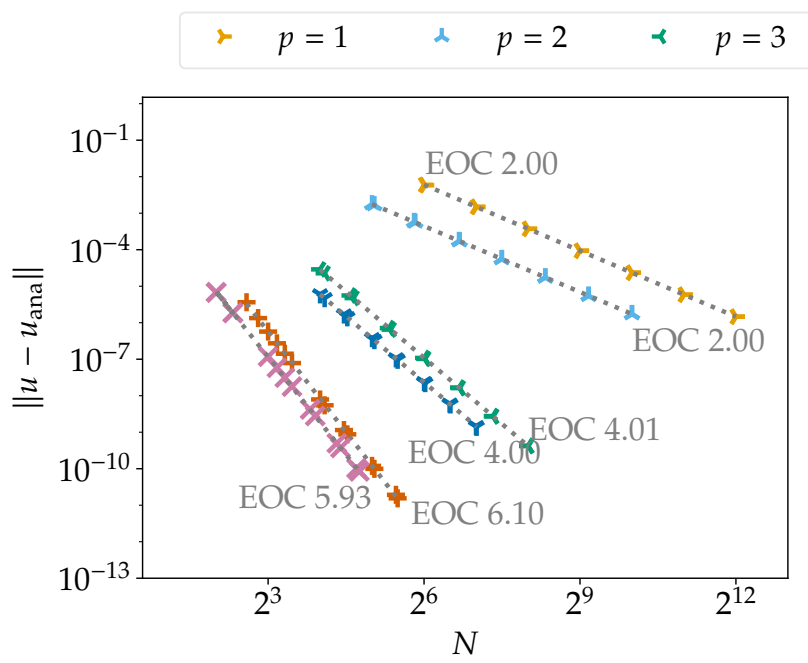

(c) Continuous Galerkin methods, $D_{2}=D_{1}^{2}$.

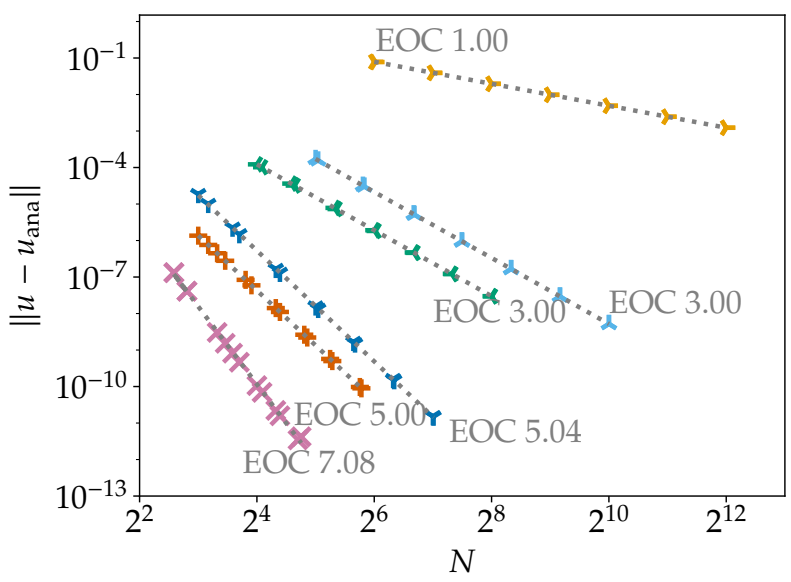

(e) Discontinuous Galerkin methods, $D_{2}=D_{1}^{2}$.

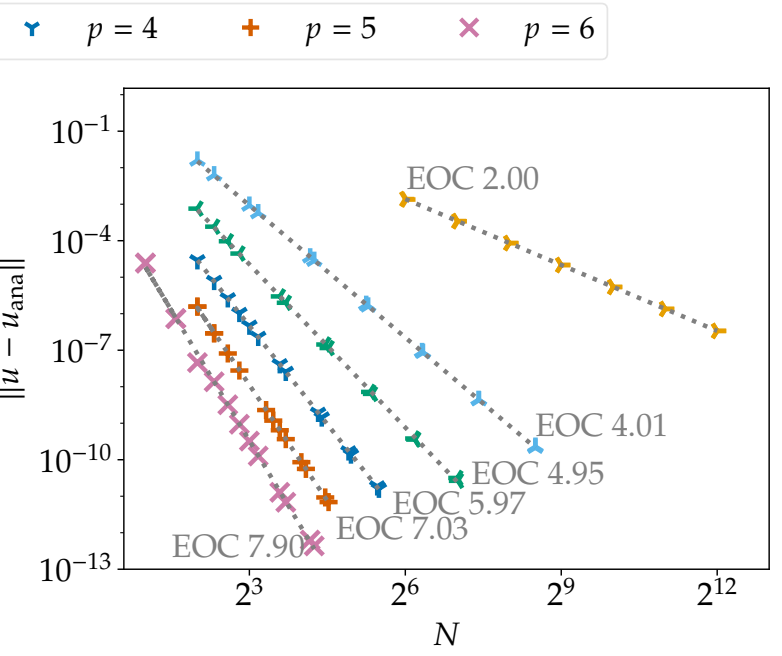

(d) CG methods, narrow-stencil $D_{2}$.

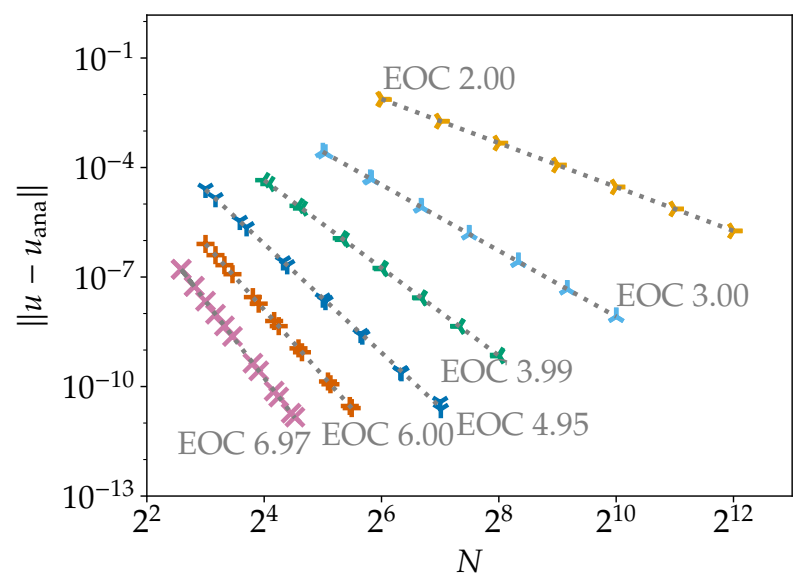

(f) DG methods, $D_{2}=D_{1,+} D_{1,-}$.

Figure 1: Convergence results of the spatial semidiscretizations (4.5) for the manufactured solution (4.8) of the BBM equation. All of these semidiscretizations conserve the linear and quadratic invariants (4.3) of the BBM equation (4.1). 


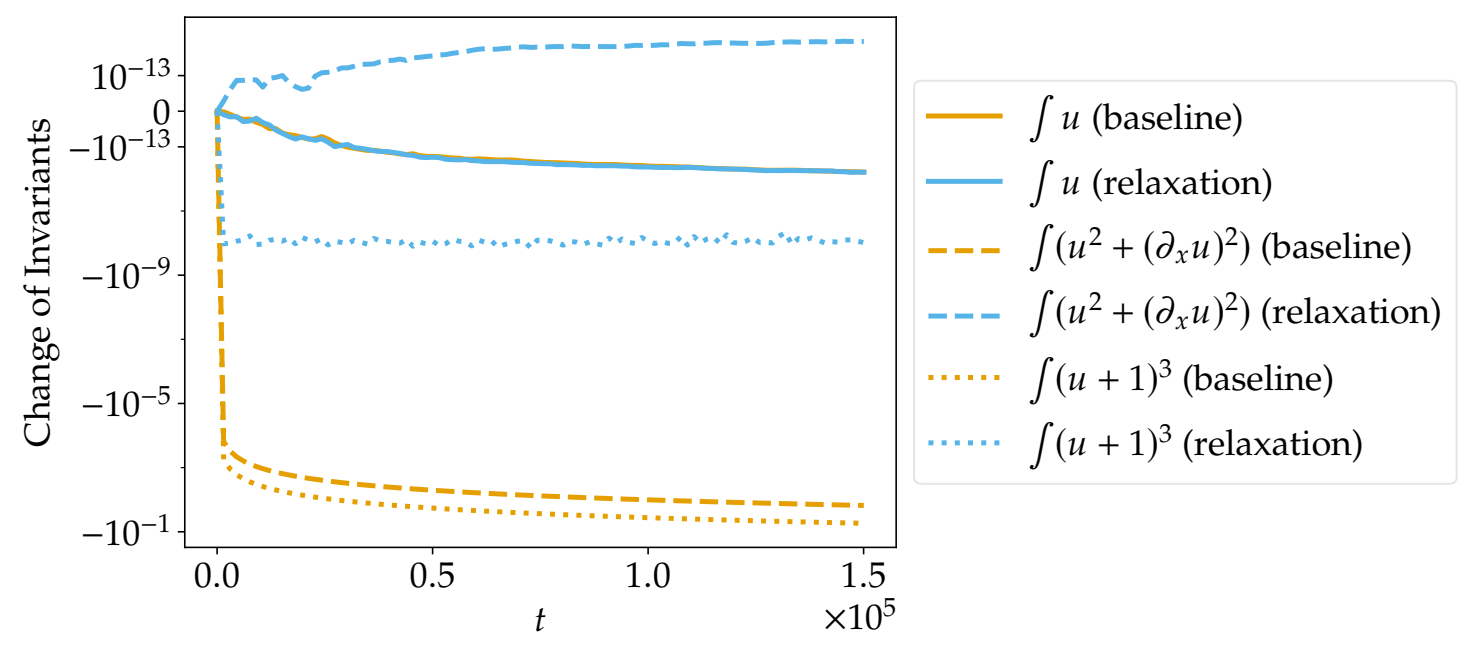

Figure 2: Temporal change in the invariants computed using RK4 with and without relaxation to preserve the quadratic invariant (4.3b) for energy-conservative Fourier collocation semidiscretizations of the traveling wave solution (4.9) of the BBM equation (4.1).

with periodic boundary conditions, which can also be written as

$$
\partial_{t} u(t, x)+\partial_{x} f(u(t, x))+\left(\mathrm{I}-\partial_{x, P}^{2}\right)^{-1} \partial_{x} u(t, x)=0,
$$

where $\left(\mathrm{I}-\partial_{x, P}^{2}\right)^{-1}$ is the inverse of the elliptic operator $\mathrm{I}-\partial_{x}^{2}$ with periodic boundary conditions. The functionals

$$
\begin{aligned}
& J_{1}^{\mathrm{FW}}(u)=\int_{x_{\text {min }}}^{x_{\max }} u, \\
& J_{2}^{\mathrm{FW}}(u)=\int_{x_{\min }}^{x_{\max }}\left(u-\partial_{x}^{2} u\right), \\
& J_{3}^{\mathrm{FW}}(u)=\int_{x_{\text {min }}}^{x_{\text {max }}} u^{2},
\end{aligned}
$$

are invariants of solutions. In the following, we will construct numerical methods that conserve all invariants (4.12).

\subsubsection{Conservative numerical methods}

The form of the nonlinearity is very similar to the BBM equation. The only difference is the additional pre-multiplication by the elliptic operator $\mathrm{I}-\partial_{x}^{2}$, which results in different invariants. Hence, basically the same split form

$$
\partial_{t} \boldsymbol{u}+\frac{1}{3} D_{1} \boldsymbol{u}^{2}+\frac{1}{3} \boldsymbol{u} D_{1} \boldsymbol{u}+\left(\mathrm{I}-D_{2}\right)^{-1} D_{1} \boldsymbol{u}=\mathbf{0}
$$

for $q=2$ can be used to conserve the invariants (4.12). For general $q \in \mathbb{N}$, similar splittings can be used [80, Section 4.5]. By applying the relaxation approach to enforce conservation of $J_{3}^{\mathrm{FW}}$ in time, we obtain a fully-discrete scheme that conserves all three invariants (4.12).

Theorem 4.4. If $D_{1}$ is a periodic first-derivative SBP operator with diagonal mass matrix $M$ and $D_{2}$ is a periodic second-derivative SBP operator, then the semidiscretization (4.13) conserves the linear invariants (4.12a) and (4.12b) of the Fornberg-Whitham equation (4.10) with $q=2$. If $D_{1} \mathcal{E} D_{2}$ commute, the quadratic invariant $(4.12 \mathrm{c})$ is also conserved. 
Proof. The first invariant (total mass) is conserved, since

$$
\begin{aligned}
\mathbf{1}^{T} M \partial_{t} \boldsymbol{u} & =-\frac{1}{3} \mathbf{1}^{T} M D_{1} \boldsymbol{u}^{2}-\frac{1}{3} \mathbf{1}^{T} M \boldsymbol{u} D_{1} \boldsymbol{u}-\mathbf{1}^{T} M\left(\mathrm{I}-D_{2}\right)^{-1} D_{1} \boldsymbol{u} \\
& =-\frac{1}{3} \mathbf{1}^{T} M D_{1} \boldsymbol{u}^{2}-\frac{1}{3} \boldsymbol{u}^{T} M D_{1} \boldsymbol{u}-\mathbf{1}^{T} M D_{1} \boldsymbol{u} \\
& =-\frac{1}{6} \boldsymbol{u}^{T} M D_{1} \boldsymbol{u}+\frac{1}{6} \boldsymbol{u}^{T} D_{1}^{T} M \boldsymbol{u}=0
\end{aligned}
$$

where we have used that $M$ is diagonal and have applied Lemma 2.28 in the second line. Lemma 2.27 has been applied in the third line. The conservation of the second invariant can be obtained similarly by applying Lemma 2.27 , resulting in

$$
\mathbf{1}^{T} M\left(\mathrm{I}-D_{2}\right) \partial_{t} \boldsymbol{u}=-\frac{1}{3} \mathbf{1}^{T} M\left(\mathrm{I}-D_{2}\right) D_{1} \boldsymbol{u}^{2}-\frac{1}{3} \mathbf{1}^{T} M\left(\mathrm{I}-D_{2}\right) \boldsymbol{u} D_{1} \boldsymbol{u}-\mathbf{1}^{T} M D_{1} \boldsymbol{u}=0 .
$$

To prove conservation of the third invariant (total energy), compute

$$
\begin{aligned}
\boldsymbol{u}^{T} M \partial_{t} \boldsymbol{u} & =-\frac{1}{3} \boldsymbol{u}^{T} M D_{1} \boldsymbol{u}^{2}-\frac{1}{3} \boldsymbol{u}^{T} M \boldsymbol{u} D_{1} \boldsymbol{u}-\boldsymbol{u}^{T} M\left(\mathrm{I}-D_{2}\right)^{-1} D_{1} \boldsymbol{u} \\
& =-\frac{1}{3} \boldsymbol{u}^{T} M D_{1} \boldsymbol{u}^{2}-\frac{1}{3}\left(\boldsymbol{u}^{2}\right)^{T} M D_{1} \boldsymbol{u}=0
\end{aligned}
$$

where Lemma 2.29 has been applied in the second line. Here, we need that $D_{1} \& D_{2}$ commute.

To test conservation, we use a smooth traveling wave solution with speed $c=1.2$ computed numerically using the Petviashvili method [77] in the periodic domain $[-80,80]$. As expected the scheme conserves all linear and the chosen nonlinear invariant up to roundoff errors.

\subsubsection{Convergence study in space}

To study convergence, the method of manufactured solutions is applied, again with the prescribed solution (4.8) with periodic boundary conditions. The results are shown in Figure 3.

Here, central finite difference methods with order of accuracy $p$ yield an EOC between $p-1 / 2$ and $p$. For other test problems such as traveling wave profiles, the EOC is closer to $p$. The results for wide-stencil and narrow-stencil second-derivative operators are similar but the narrow-stencil operators result in smaller errors (smaller by less than an order of magnitude).

In contrast to results for the BBM equation, the choice of the second-derivative operator does not influence the EOC for CG methods significantly. Both wide and narrow-stencil operators $D_{2}$ yield EOC between $p+1 / 2$ and $p+1$ for odd polynomial degrees $p$ and EOC $\approx p$ for even $p$. However, only the wide-stencil operators conserve the quadratic invariant.

A similar observation can be made for nodal discontinuous Galerkin methods. There, both types of operators $D_{2}$ yield EOC $\approx p+1$ for even polynomial degrees $p$ and EOC $\approx p$ for odd $p$. Again, only the wide-stencil operators conserve the quadratic invariant.

\subsection{Camassa-Holm equation}

Consider the Camassa-Holm equation [21]

$$
\begin{aligned}
\left(\mathrm{I}-\partial_{x}^{2}\right) \partial_{t} u(t, x)+\partial_{x}\left(\frac{3}{2} u(t, x)^{2}-\frac{1}{2}\left(\partial_{x} u(t, x)\right)^{2}-u(t, x) \partial_{x}^{2} u(t, x)\right) & =0 \\
t & \in(0, T), x \in\left(x_{\min }, x_{\max }\right) \\
u(0, x) & =u^{0}(x) \\
x & \in\left[x_{\min }, x_{\max }\right]
\end{aligned}
$$




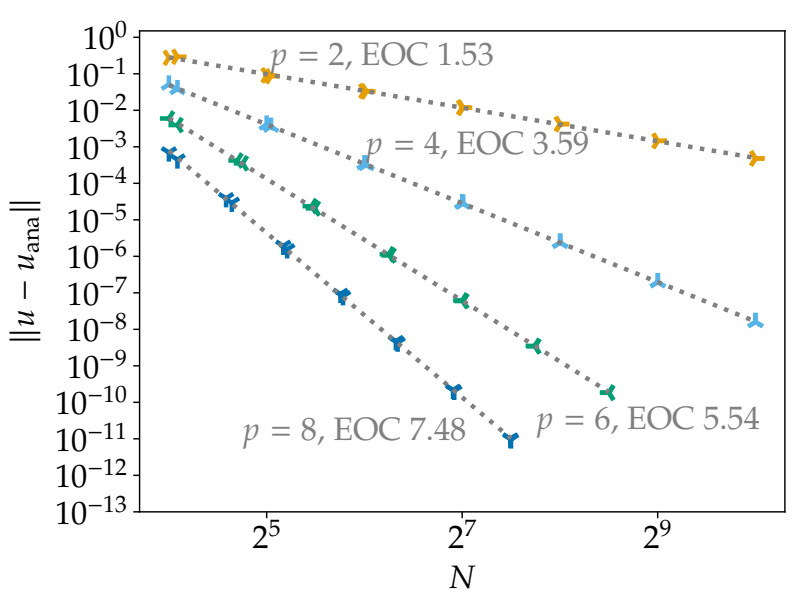

(a) Finite difference methods, wide stencil $D_{2}=D_{1}^{2}$.

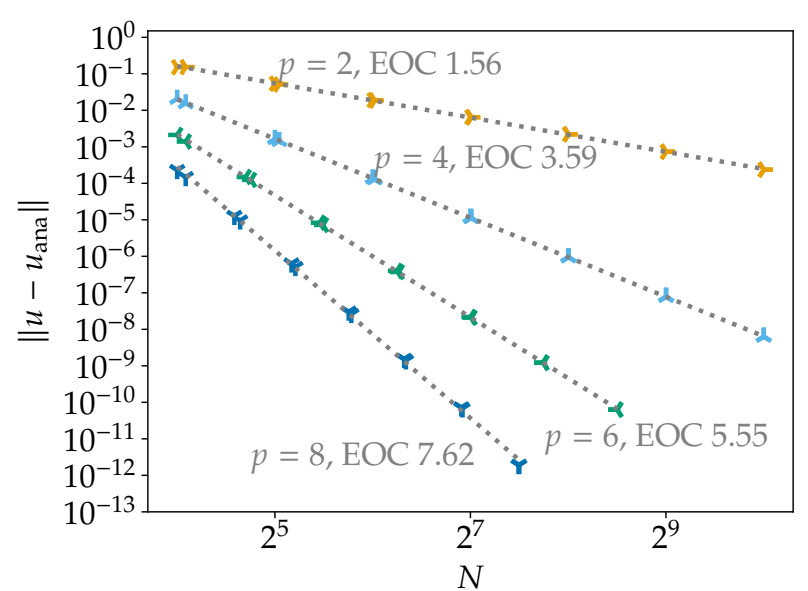

(b) Finite difference methods, narrow stencil $D_{2}$.

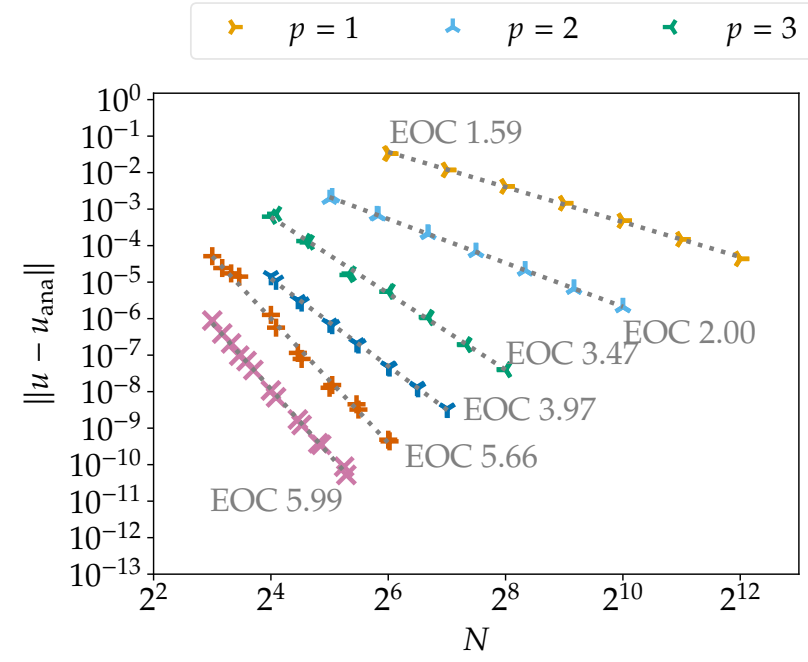

` $p=4+p=5 \quad \times \quad p=6$

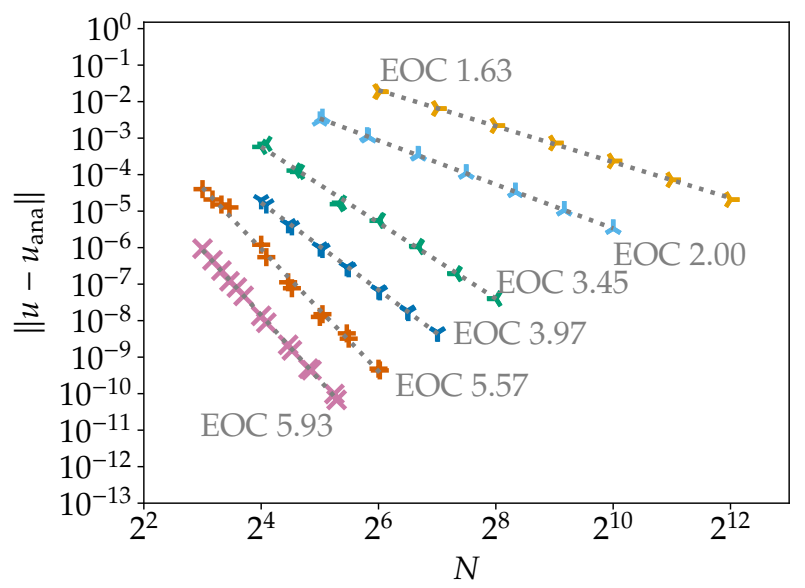

(c) Continuous Galerkin methods, $D_{2}=D_{1}^{2}$.

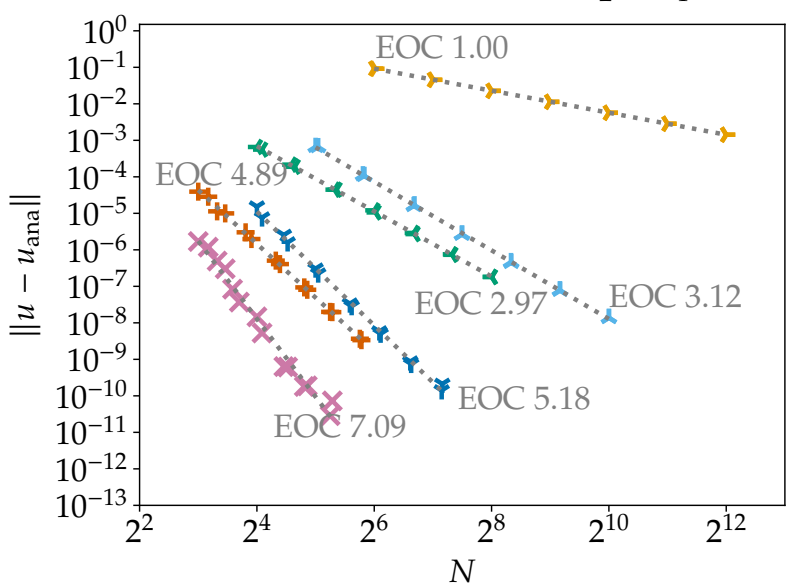

(e) Discontinuous Galerkin methods, $D_{2}=D_{1}^{2}$.

(d) Continuous Galerkin methods, narrow stencil $D_{2}$.

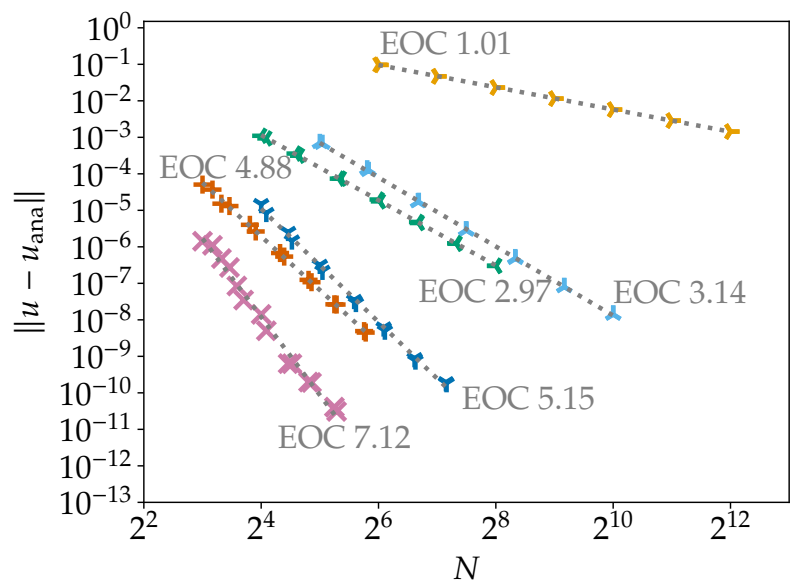

(f) Discontinuous Galerkin methods, $D_{2}=D_{1,+} D_{1,-}$.

Figure 3: Convergence results of the spatial semidiscretizations (4.13) for the manufactured solution (4.8) of the FW equation. All of these semidiscretizations conserve the linear invariants (4.12) of the FW equation (4.10). The FD methods and the Galerkin methods with wide stencil $D_{2}$ conserve the quadratic invariant as well. 
with periodic boundary conditions, which can also be written as

$$
\partial_{t} u+\left(\mathrm{I}-\partial_{x, P}^{2}\right)^{-1}\left(\partial_{x} u^{2}+u \partial_{x} u-\alpha \partial_{x}\left(u \partial_{x}^{2} u\right)-(1-\alpha) \partial_{x}^{2}\left(u \partial_{x} u\right)-(2 \alpha-1)\left(\partial_{x} u\right)\left(\partial_{x}^{2} u\right)\right)=0,
$$

where $\left(\mathrm{I}-\partial_{x, P}^{2}\right)^{-1}$ is the inverse of the elliptic operator $\mathrm{I}-\partial_{x}^{2}$ with periodic boundary conditions and $\alpha \in \mathbb{R}$ is a parameter determining the split form. The splitting of the quadratic term $\frac{3}{2} \partial_{x} u^{2}$ is the same as for Burgers' equation (2.45). The one-parameter split form of the third-derivative terms has been constructed using similar manipulations.

Important invariants of solutions are

$$
\begin{aligned}
& J_{1}^{\mathrm{CH}}(u)=\int_{x_{\text {min }}}^{x_{\text {max }}} u, \\
& J_{2}^{\mathrm{CH}}(u)=\frac{1}{2} \int_{x_{\text {min }}}^{x_{\text {max }}}\left(u^{2}+\left(\partial_{x} u\right)^{2}\right)=\frac{1}{2} \int_{x_{\text {min }}}^{x_{\max }} u\left(\mathrm{I}-\partial_{x}^{2}\right) u, \\
& J_{3}^{\mathrm{CH}}(u)=\int_{x_{\text {min }}}^{x_{\text {max }}}\left(u^{3}+u\left(\partial_{x} u\right)^{2}\right) .
\end{aligned}
$$

In the following, we will construct numerical methods that conserve the linear (4.19a) and quadratic (4.19b) invariants but not necessarily the cubic invariant (4.19c).

\subsubsection{Conservative numerical methods}

Using the splitting as in (4.18), semidiscretizations that conserve the linear and quadratic invariant can be obtained as

$$
\partial_{t} \boldsymbol{u}+\left(\mathrm{I}-D_{2, a}\right) \not \boldsymbol{C}_{1} \boldsymbol{u}^{2}+\boldsymbol{u} D_{1} \boldsymbol{u}-\alpha D_{1}\left(\boldsymbol{u} D_{2, b} \boldsymbol{u}\right)-(1-\alpha) D_{2, b}\left(\boldsymbol{u} D_{1} \boldsymbol{u}\right)-(2 \alpha-1)\left(D_{1} \boldsymbol{u}\right) D_{2, b} \boldsymbol{u}=\mathbf{0} \text {. }
$$

Theorem 4.5. If $D_{1}$ is a periodic first-derivative SBP operator with diagonal mass matrix $M$ and $D_{2, a}, D_{2, b}$ are periodic second-derivative SBP operator, then the semidiscretization (4.20) conserves the quadratic invariant (4.19b) of (4.17). If $D_{1}$ and $D_{2, b}$ commute or $\alpha=1 / 2$, the linear invariant (4.19a) is conserved as well.

Proof. The rate of change of the linear invariant (4.19a) is

$$
\begin{aligned}
& \frac{\mathrm{d}}{\mathrm{d} t} J_{1}^{\mathrm{CH}}(\boldsymbol{u})=\frac{\mathrm{d}}{\mathrm{d} t} \mathbf{1}^{T} M \boldsymbol{u}=\mathbf{1}^{T} M \partial_{t} \boldsymbol{u}=-\mathbf{1}^{T} M\left(\mathrm{I}-D_{2, a}\right)^{-1}\left(\left(\mathrm{I}-D_{2, a}\right) \partial_{t} \boldsymbol{u}\right) \\
= & -\mathbf{1}^{T} M\left(D_{1} \boldsymbol{u}^{2}+\boldsymbol{u} D_{1} \boldsymbol{u}-\alpha D_{1}\left(\boldsymbol{u} D_{2, b} \boldsymbol{u}\right)-(1-\alpha) D_{2, b}\left(\boldsymbol{u} D_{1} \boldsymbol{u}\right)-(2 \alpha-1)\left(D_{1} \boldsymbol{u}\right) D_{2, b} \boldsymbol{u}\right) \\
= & (2 \alpha-1) \boldsymbol{u}^{T} D_{1}^{T} M D_{2, b} \boldsymbol{u},
\end{aligned}
$$

where Lemma 2.28 has been used in the second line and Lemma 2.27 has been used in the last step. If $D_{1}$ and $D_{2, b}$ commute,

$$
\frac{\mathrm{d}}{\mathrm{d} t} J_{1}^{\mathrm{CH}}(\boldsymbol{u})=(2 \alpha-1) \frac{1}{2} \boldsymbol{u}^{T} D_{1}^{T} D_{2, b}^{T} M \boldsymbol{u}-(2 \alpha-1) \frac{1}{2} \boldsymbol{u}^{T} M D_{1} D_{2, b} \boldsymbol{u}=0 .
$$

Since $\mathrm{I}-D_{2, a}$ is a symmetric operator, the semidiscrete rate of change of the quadratic invariant $(4.19 b)$ is

$$
\begin{aligned}
& \frac{\mathrm{d}}{\mathrm{d} t} J_{2}^{\mathrm{CH}}(\boldsymbol{u})=\frac{1}{2} \frac{\mathrm{d}}{\mathrm{d} t} \boldsymbol{u}^{T} M\left(\mathrm{I}-D_{2, a}\right) \boldsymbol{u}=\boldsymbol{u}^{T} M\left(\mathrm{I}-D_{2, a}\right) \partial_{t} \boldsymbol{u} \\
= & -\boldsymbol{u}^{T} M\left(D_{1} \boldsymbol{u}^{2}+\boldsymbol{u} D_{1} \boldsymbol{u}-\alpha D_{1}\left(\boldsymbol{u} D_{2, b} \boldsymbol{u}\right)-(1-\alpha) D_{2, b}\left(\boldsymbol{u} D_{1} \boldsymbol{u}\right)-(2 \alpha-1)\left(D_{1} \boldsymbol{u}\right) D_{2, b} \boldsymbol{u}\right) \\
= & \alpha \boldsymbol{u}^{T} M\left(D_{1}\left(\boldsymbol{u} D_{2, b} \boldsymbol{u}\right)+\left(D_{1} \boldsymbol{u}\right) D_{2, b} \boldsymbol{u}\right)+(1-\alpha) \boldsymbol{u}^{T} M\left(D_{2, b}\left(\boldsymbol{u} D_{1} \boldsymbol{u}\right)-\left(D_{1} \boldsymbol{u}\right) D_{2, b} \boldsymbol{u}\right)=0 .
\end{aligned}
$$


Remark 4.6. A dissipative LDG method based on exact integration instead of split forms and equivalents of $D_{2, a}=D_{2, b}=D_{1,-} D_{1,+}$ or $D_{2, a}=D_{2, b}=D_{1,+} D_{1,-}$ has been proposed in [106]. The split form semidiscretization (4.20) with $\alpha=1$ and $D_{2, a}=D_{2, b}=D_{1}^{2}$ has been used in [63] for second order FD methods and in [64] for DG methods. The same split form with $D_{2, a}=D_{2, b}$ has been used in [50] for Fourier collocation methods. Without applying a splitting, a Fourier collocation method conserving the cubic invariant (4.19c) has been used in [20].

Remark 4.7. Similarly to the FW equation and in contrast to the BBM equation, an additional restriction on the first- and second-derivative operators arises for the $\mathrm{CH}$ equation: They need to commute to conserve the quadratic invariant (4.19b) unless the splitting parameter is chosen as $\alpha=1 / 2$. The existence of such a splitting and the potential possibility to use different secondderivative operators provides interesting possibilities. In preliminary numerical studies, using $D_{2, a}=D_{2, b}$ seems to be a good choice.

To verify the conservation properties of the semidiscretization (4.20), we used a smooth traveling wave solution with speed $c=1.2$ computed numerically using the Petviashvili method in the periodic domain $[-40,40]$. This traveling wave solution has been computed for the PDE

$$
\left(\mathrm{I}-\partial_{x}^{2}\right) \partial_{t} u(t, x)+2 \kappa \partial_{x} u(t, x)+\partial_{x}\left(\frac{3}{2} u(t, x)^{2}-\frac{1}{2}\left(\partial_{x} u(t, x)\right)^{2}-u(t, x) \partial_{x}^{2} u(t, x)\right)=0,
$$

which can be transformed to a solution of the $\mathrm{CH}$ equation (with $\kappa=0$ ) by the transformation

$$
x \rightarrow x+\kappa t, \quad u \rightarrow u+\kappa .
$$

\subsubsection{Convergence study in space}

For the following convergence study, the method of manufactured solutions is applied to (4.8) with periodic boundary conditions. The results are shown in Figure 4.

Similarly to the BBM equation, central finite difference methods with order of accuracy $p$ yield an $\mathrm{EOC} \approx p$. The results for wide-stencil and narrow-stencil second-derivative operators are again similar and the narrow-stencil operators result in smaller errors (less than an order of magnitude).

For CG methods, the choice of the second-derivative operator does not influence the EOC significantly, similarly to the FW equation and in contrast to the BBM equation. Both wide and narrow-stencil operators $D_{2}$ yield EOC $\approx p+1$ for odd polynomial degrees $p$ and EOC $\approx p$ for even $p$. In contrast to the other examples discussed before, the error depends on the parity of the number of elements for odd polynomial degrees and the wide stencil second-derivative operator $D_{2}=D_{1}^{2}$. In these cases, the error is smaller if an odd number of elements is used. By just adding one element to go from even $N$ to odd $N$, the error can be reduced up to an order of magnitude. The method using $p=3$ results in approximately the same error as the one for $p=4$ if $N$ is odd while its error is up to an order of magnitude bigger for even $N$. Such a behavior cannot be observed for the narrow-stencil operator or even polynomial degrees.

Similar observations can be made for nodal discontinuous Galerkin methods. There, both types of operators $D_{2}$ yield EOC $\approx p+1$ for even polynomial degrees $p$ and EOC $\approx p$ for odd $p$. The only exception to this rule is the narrow-stencil second-derivative operator $D_{2}=D_{1,+} D_{1,-}$ with $p=1$, which doesn't converge. Since this phenomenon occurs only for this specific parameter combination and convergence can be obtained, e.g. for $\alpha=1$, it is not studied in detail here. As for CG methods, there is a dependence of the error on the parity of the number of elements. For DG methods, this dependence manifests only for wide-stencil operators $D_{2}=D_{1}^{2}$ and even polynomial degrees (while it occurs for odd polynomial degrees for CG methods). Again, the error is up to an order of magnitude smaller for odd $N$. 


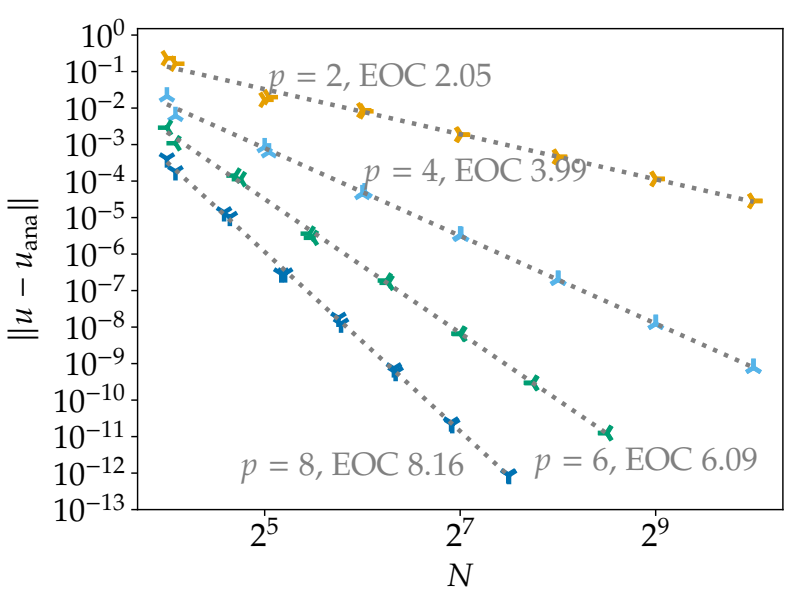

(a) Finite difference methods, wide stencil $D_{2}=D_{1}^{2}$.

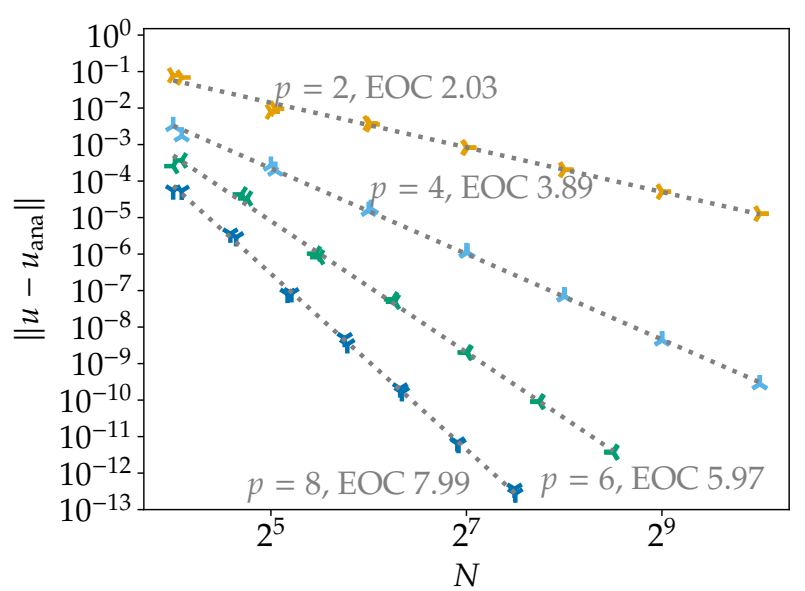

(b) Finite difference methods, narrow stencil $D_{2}$.

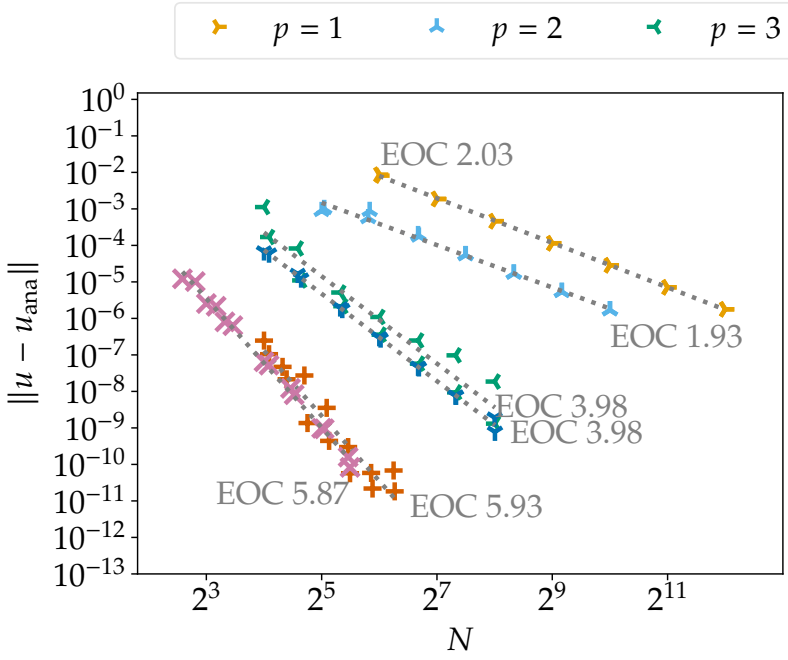

ч $p=4+p=5 \quad \times \quad p=6$

(c) Continuous Galerkin methods, $D_{2}=D_{1}^{2}$.

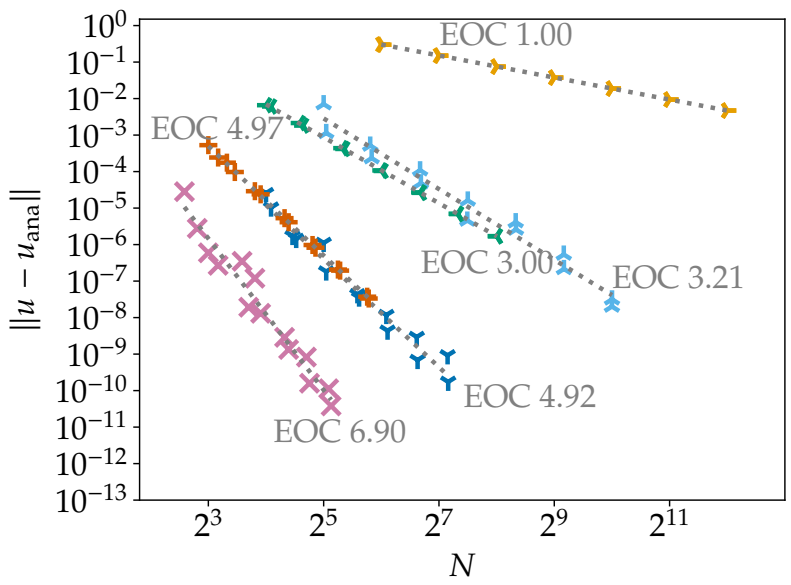

(e) Discontinuous Galerkin methods, $D_{2}=D_{1}^{2}$.

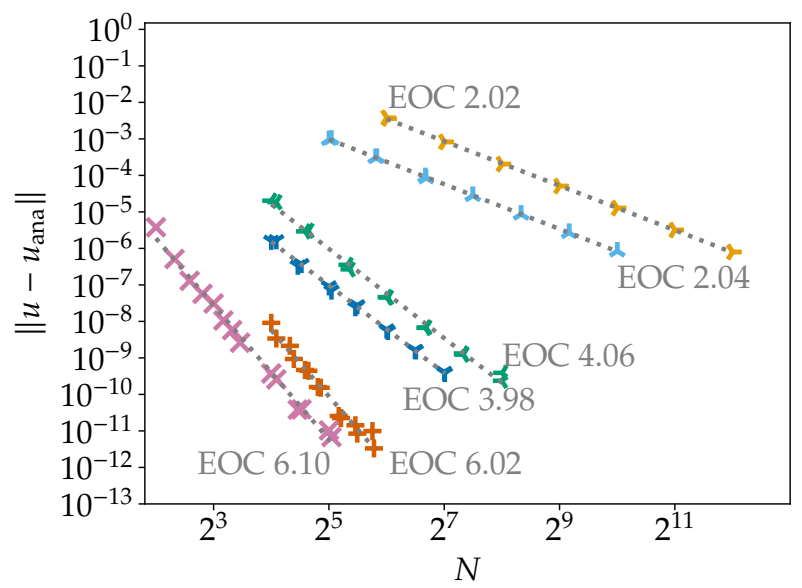

(d) Continuous Galerkin methods, narrow stencil $D_{2}$.

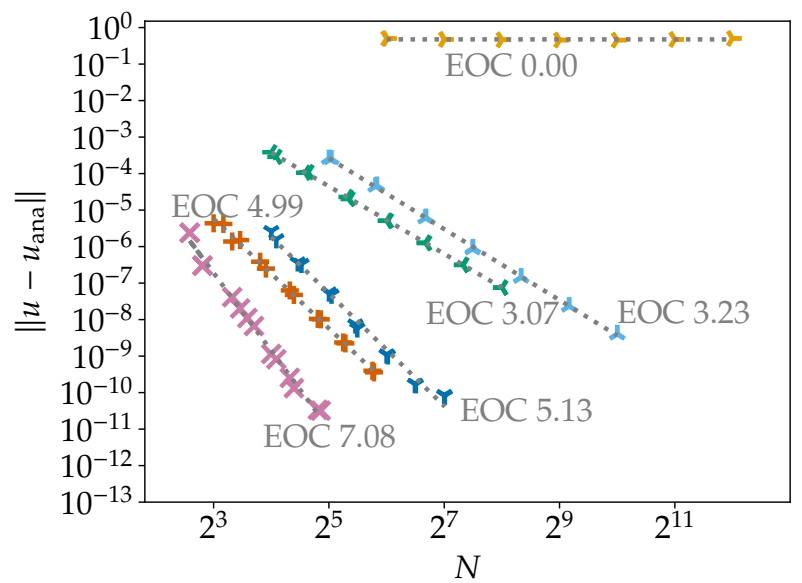

(f) Discontinuous Galerkin methods, $D_{2}=D_{1,+} D_{1,-}$.

Figure 4: Convergence results of the spatial semidiscretizations (4.20) with $\alpha=1 / 2$ and $D_{2, a}=D_{2, b}=D_{2}$ for the manufactured solution (4.8) of the $\mathrm{CH}$ equation. All of these semidiscretizations conserve the linear and quadratic invariants (4.19) of the $\mathrm{CH}$ equation (4.17). 


\subsection{Degasperis-Procesi equation}

Consider the Degasperis-Procesi equation [31]

$$
\begin{aligned}
& \left(\mathrm{I}-\partial_{x}^{2}\right) \partial_{t} u(t, x)+4 \partial_{x} f(u(t, x))-\partial_{x}^{3} f(u(t, x))=0, \quad t \in(0, T), x \in\left(x_{\min }, x_{\max }\right), \\
& u(0, x)=u^{0}(x), \quad x \in\left[x_{\min }, x_{\max }\right], \\
& f(u)=\frac{u^{2}}{2}
\end{aligned}
$$

with periodic boundary conditions, which can also be written as

$$
\partial_{t} u(t, x)+\left(\mathrm{I}-\partial_{x, P}^{2}\right)^{-1}\left(4 \mathrm{I}-\partial_{x}^{2}\right) \partial_{x} f(u(t, x))=0,
$$

where $\left(\mathrm{I}-\partial_{x, P}^{2}\right)^{-1}$ is the inverse of the elliptic operator $\mathrm{I}-\partial_{x}^{2}$ with periodic boundary conditions. The functionals

$$
\begin{aligned}
& J_{1}^{\mathrm{DP}}(u)=\int_{x_{\text {min }}}^{x_{\text {max }}}\left(u-\partial_{x}^{2} u\right), \\
& \left.J_{2}^{\mathrm{DP}}(u)=\frac{1}{2} \int_{x_{\text {min }}}^{x_{\text {max }}}\left(\left(u-\partial_{x}^{2} u\right) v\right)\right), \quad v=\left(4 \mathrm{I}-\partial_{x, P}^{2}\right)^{-1} u, \\
& J_{3}^{\mathrm{DP}}(u)=\int_{x_{\text {min }}}^{x_{\text {max }}} u^{3},
\end{aligned}
$$

are invariants of solutions. We would like to emphasize that (4.17) and (4.26) can be written using the same linear and nonlinear terms multiplied by different constant coefficients. These result in different invariants and other split forms available/necessary for conservative methods. In the following, we will construct numerical methods that conserve the linear (4.28a) and quadratic (4.28b) invariants but not necessarily the cubic invariant (4.28c).

\subsubsection{Conservative numerical methods}

The rate of change of the quadratic invariant (4.28b) results basically in the same integral terms as the energy rate of Burgers' equation (2.43). Hence, the same kind of splitting can be used to obtain semidiscretizations

$$
\partial_{t} \boldsymbol{u}+\frac{1}{3}\left(\mathrm{I}-D_{2}\right)^{-1}\left(4 \mathrm{I}-D_{2}\right)\left(D_{1} \boldsymbol{u}^{2}+\boldsymbol{u} D_{1} \boldsymbol{u}\right)=\mathbf{0}
$$

that conserve the linear and quadratic invariant.

Theorem 4.8. If $D_{1}$ is a periodic first-derivative SBP operator with diagonal mass matrix $M$ and $D_{2}$ is a periodic second-derivative SBP operator, then the semidiscretization (4.29) conserves the invariants (4.28a) and (4.28b) of (4.26).

Proof. The linear invariant (4.28a) is conserved since

$$
\begin{aligned}
\frac{\mathrm{d}}{\mathrm{d} t} J_{1}^{\mathrm{DP}}(\boldsymbol{u})=\frac{\mathrm{d}}{\mathrm{d} t} \mathbf{1}^{T} M\left(\mathrm{I}-D_{2}\right) \boldsymbol{u}=\mathbf{1}^{T} M\left(\mathrm{I}-D_{2}\right) \partial_{t} \boldsymbol{u}= & -\frac{1}{3} \mathbf{1}^{T} M\left(4 \mathrm{I}-D_{2}\right)\left(D_{1} \boldsymbol{u}^{2}+\boldsymbol{u} D_{1} \boldsymbol{u}\right) \\
& =-\frac{1}{3} \mathbf{1}^{T} M\left(4 \mathrm{I}-D_{2}\right)\left(D_{1} \boldsymbol{u}^{2}+\boldsymbol{u} D_{1} \boldsymbol{u}\right)=0
\end{aligned}
$$

where Lemma 2.28 and Lemma 2.27 have been used.

Since I $-D_{2}$ and $4 \mathrm{I}-D_{2}$ are commuting symmetric operators, the semidiscrete rate of change of the quadratic invariant $(4.28 \mathrm{~b})$ is

$$
\begin{aligned}
& \frac{1}{2} \frac{\mathrm{d}}{\mathrm{d} t} \boldsymbol{u}^{T}\left(4 \mathrm{I}-D_{2}\right)^{-T} M\left(\mathrm{I}-D_{2}\right) \boldsymbol{u}=\boldsymbol{u}^{T}\left(4 \mathrm{I}-D_{2}\right)^{-T} M\left(\mathrm{I}-D_{2}\right) \partial_{t} \boldsymbol{u} \\
& \quad=-\frac{1}{3} \boldsymbol{u}^{T}\left(4 \mathrm{I}-D_{2}\right)^{-T} M\left(4 \mathrm{I}-D_{2}\right)\left(D_{1} \boldsymbol{u}^{2}+\boldsymbol{u} D_{1} \boldsymbol{u}\right)=-\frac{1}{3} \boldsymbol{u}^{T} M\left(D_{1} \boldsymbol{u}^{2}+\boldsymbol{u} D_{1} \boldsymbol{u}\right)=0 .
\end{aligned}
$$


Remark 4.9. The split form discretization (4.29) is used in [105] for Fourier collocation methods. Substituting the split form in (4.29) by the conservative form results in a semidiscretization that conserves both the linear invariant (4.28a) and the cubic invariant (4.28c). This has been used for Fourier collocation methods in [20].

Remark 4.10. Conservation of the quadratic invariant $(4.28 \mathrm{~b})$ yields the estimate $\|\boldsymbol{u}(t)\|_{M}^{2} \leq$ $4\left\|\boldsymbol{u}^{0}\right\|_{M}^{2}$, cf. [105]. Indeed, setting $\boldsymbol{v}=\left(4 \mathrm{I}-D_{2}\right)^{-1} \boldsymbol{u}$,

$$
\|\boldsymbol{u}\|_{M}^{2}=\left\|\left(4 \mathrm{I}-D_{2}\right) \boldsymbol{v}\right\|_{M}^{2}=16\|\boldsymbol{v}\|_{M}^{2}+8\|\boldsymbol{v}\|_{A_{2}}^{2}+\left\|D_{2} \boldsymbol{v}\right\|_{M}^{2} .
$$

Using

$$
J_{2}^{\mathrm{DP}}(\boldsymbol{u})=\boldsymbol{v}^{T} M\left(\mathrm{I}-D_{2}\right) \boldsymbol{u}=\boldsymbol{v}^{T} M\left(\mathrm{I}-D_{2}\right)\left(4 \mathrm{I}-D_{2}\right) \boldsymbol{v}=4\|\boldsymbol{v}\|_{M}^{2}+5\|\boldsymbol{v}\|_{A_{2}}^{2}+\left\|D_{2} \boldsymbol{v}\right\|_{M}^{2} .
$$

yields the bounds $\|\boldsymbol{u}\|_{M}^{2} \leq 4 J_{2}^{\mathrm{DP}}(\boldsymbol{u})$ and $\|\boldsymbol{u}\|_{M}^{2} \geq J_{2}^{\mathrm{DP}}(\boldsymbol{u})$. Hence, $\|\boldsymbol{u}(t)\|_{M}^{2} \leq 4 J_{2}^{\mathrm{DP}}(\boldsymbol{u}(t))=4 J_{2}^{\mathrm{DP}}\left(\boldsymbol{u}^{0}\right) \leq$ $4\left\|\boldsymbol{u}^{0}\right\|_{M}^{2}$.

A smooth traveling wave solution with speed $c=1.2$ computed numerically using the Petviashvili method in the periodic domain $[-40,40]$ was used to verify the conservation properties of the semidiscretization (4.20). This traveling wave solution has been computed for the PDE

$$
\left(\mathrm{I}-\partial_{x}^{2}\right) \partial_{t} u(t, x)+3 \kappa \partial_{x} u(t, x)+2 \partial_{x} u(t, x)^{2}-\frac{1}{2} \partial_{x}^{3} u(t, x)^{2}=0
$$

which can be transformed to a solution of the DP equation (with $\kappa=0$ ) by the transformation

$$
x \rightarrow x+\kappa t, \quad u \rightarrow u+\kappa .
$$

\subsubsection{Convergence study in space}

For the following convergence study, the method of manufactured solutions is applied to (4.8) with periodic boundary conditions. The results are shown in Figure 5.

Similarly to the FW equation, central finite difference methods with order of accuracy $p$ yield an EOC between $p-1 / 2$ and $p$. For other test problems such as traveling wave profiles, the EOC is closer to $p$. The results for wide-stencil and narrow-stencil second-derivative operators are similar but the narrow-stencil operators result again in smaller errors (up to an order of magnitude).

As for the FW and $\mathrm{CH}$ equations and in contrast to the BBM equation, the choice of the secondderivative operator does not influence the EOC significantly for nodal continuous Galerkin methods. Both wide and narrow-stencil operators $D_{2}$ yield an EOC between $p+1 / 2$ and $p+1$ for odd polynomial degrees $p$ and EOC $\approx p$ for even $p$. For traveling wave solutions and odd polynomial degrees, the EOC is closer to $p+1$.

Similar observations can be made for nodal discontinuous Galerkin methods. There, both types of operators $D_{2}$ yield EOC $\approx p+1$ for even polynomial degrees $p$ and EOC $\approx p$ for odd $p$.

\subsection{Holm-Hone equation}

Consider the Holm-Hone equation [49]

$$
\begin{aligned}
\left(4-5 \partial_{x}^{2}+\partial_{x}^{4}\right) \partial_{t} u+u \partial_{x}^{5} u+2\left(\partial_{x} u\right) \partial_{x}^{4} u-5 u \partial_{x}^{3} u-10\left(\partial_{x} u\right) \partial_{x}^{2} u+12 u \partial_{x} u & =0, \\
t & \in(0, T), x \in\left(x_{\min }, x_{\max }\right), \\
u(0, x) & =u^{0}(x), \\
x & \in\left[x_{\min }, x_{\max }\right],
\end{aligned}
$$

with periodic boundary conditions, which can also be written as

$$
\partial_{t} u+\left(4 \mathrm{I}-5 \partial_{x, P}^{2}+\partial_{x, P}^{4}\right)^{-1}\left(\partial_{x}\left(u\left(4 \mathrm{I}-5 \partial_{x}^{2}+\partial_{x}^{4}\right) u\right)+\left(\partial_{x} u\right)\left(4 \mathrm{I}-5 \partial_{x}^{2}+\partial_{x}^{4}\right) u\right)=0,
$$




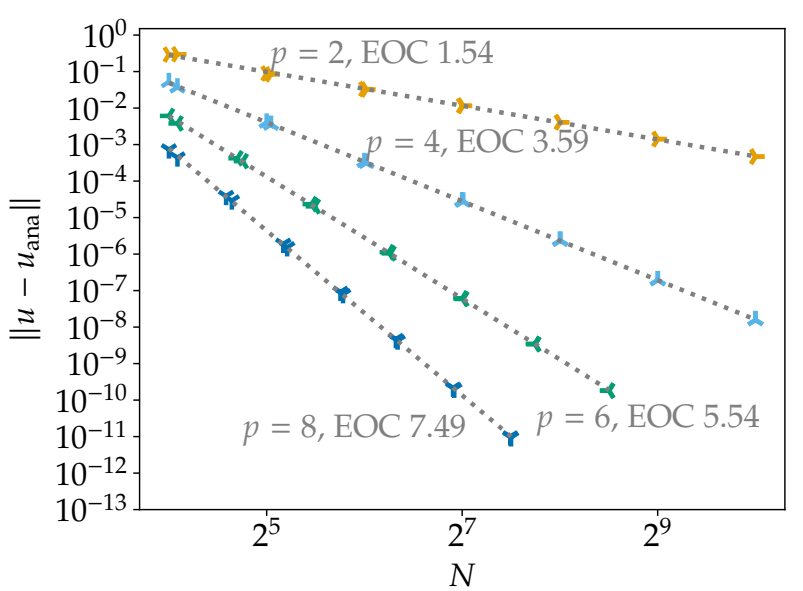

(a) Finite difference methods, wide stencil $D_{2}=D_{1}^{2}$.

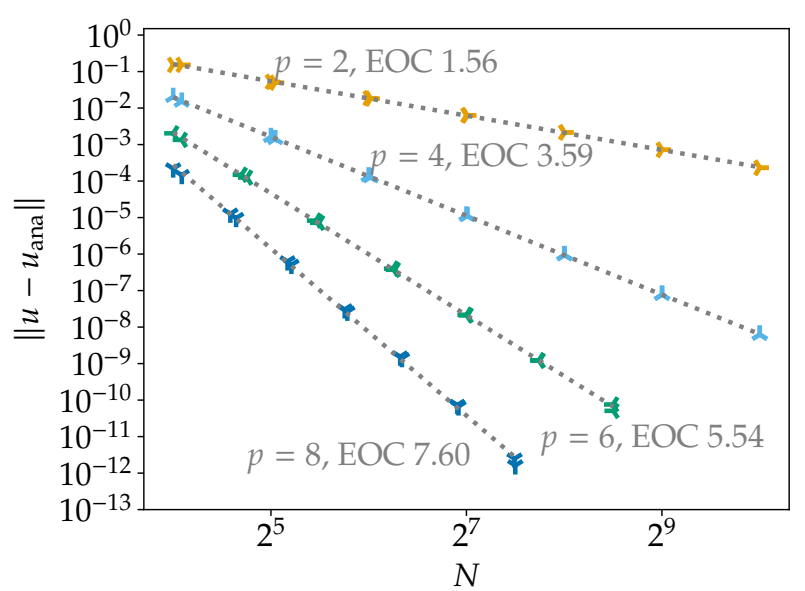

(b) Finite difference methods, narrow stencil $D_{2}$.

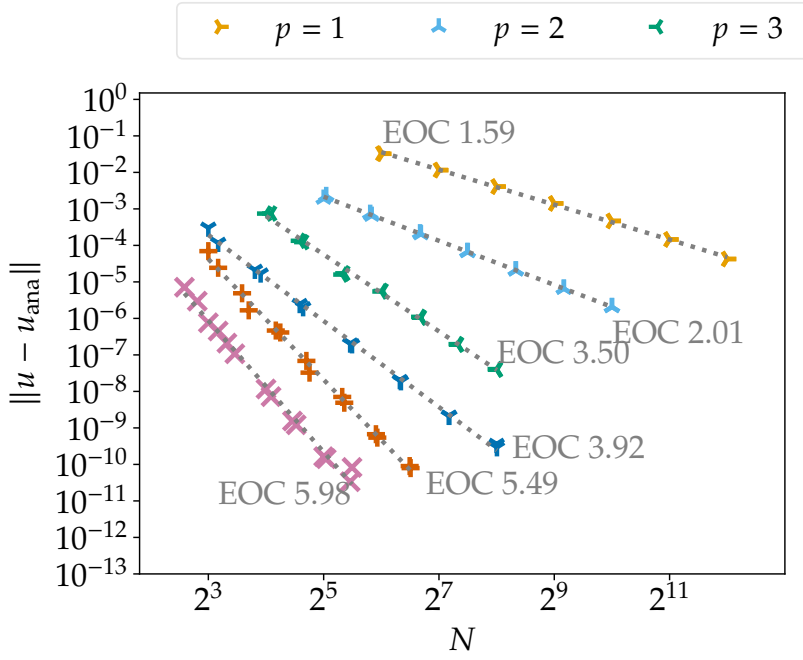

ฯ $p=4+p=5 \quad \times \quad p=6$

(c) Continuous Galerkin methods, $D_{2}=D_{1}^{2}$.

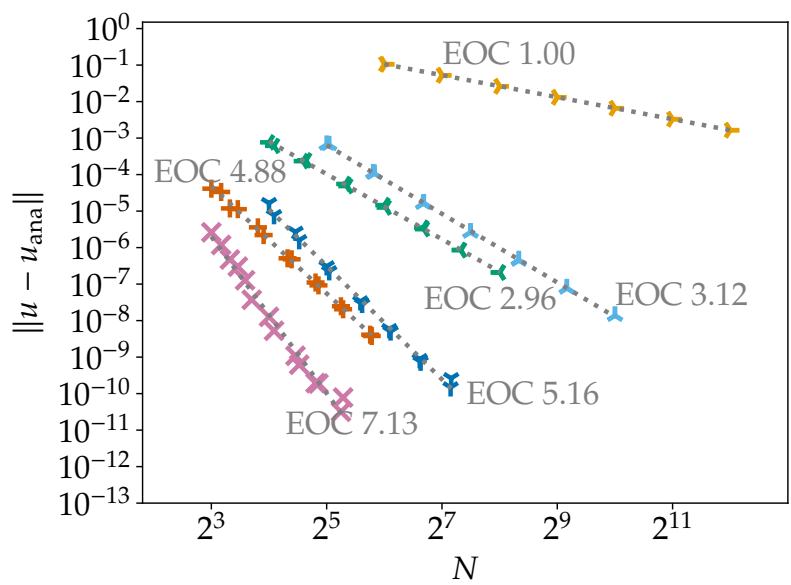

(e) Discontinuous Galerkin methods, $D_{2}=D_{1}^{2}$.

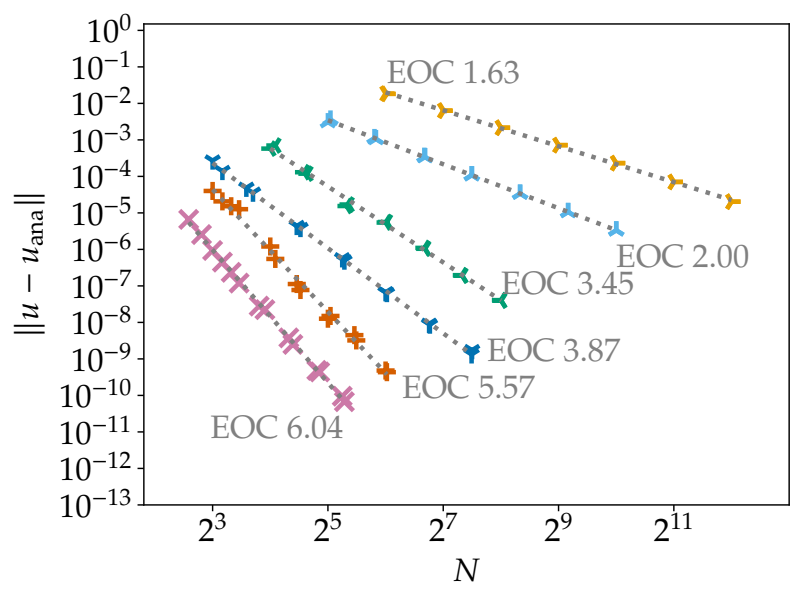

(d) Continuous Galerkin methods, narrow stencil $D_{2}$.

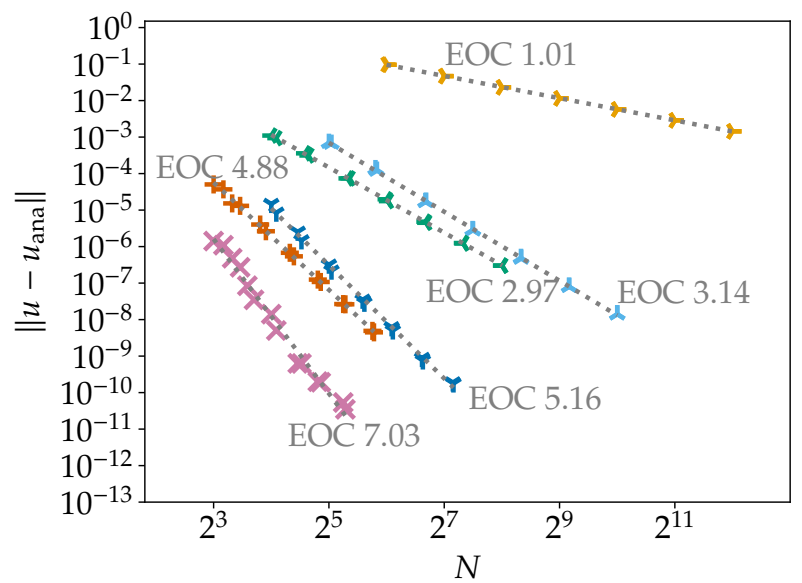

(f) Discontinuous Galerkin methods, $D_{2}=D_{1,+} D_{1,-}$.

Figure 5: Convergence results of the spatial semidiscretizations (4.29) for the manufactured solution (4.8) of the DP equation. All of these semidiscretizations conserve the linear and quadratic invariants (4.28) of the DP equation (4.26). 
where $\left(4 \mathrm{I}-5 \partial_{x, P}^{2}+\partial_{x, P}^{4}\right)^{-1}$ is the inverse of the elliptic operator $4 \mathrm{I}-5 \partial_{x}^{2}+\partial_{x}^{4}$ with periodic boundary conditions. The functionals

$$
\begin{aligned}
& J_{1}^{\mathrm{HH}}(u)=\int_{x_{\min }}^{x_{\max }} u, \\
& J_{2}^{\mathrm{HH}}(u)=\int_{x_{\min }}^{x_{\max }}\left(4 \mathrm{I}-\partial_{x}^{2}\right)\left(\mathrm{I}-\partial_{x}^{2}\right) u=\int_{x_{\min }}^{x_{\max }}\left(4 \mathrm{I}-5 \partial_{x}^{2}+\partial_{x}^{4}\right) u, \\
& J_{3}^{\mathrm{HH}}(u)=\frac{1}{2} \int_{x_{\min }}^{x_{\max }} u\left(4 \mathrm{I}-\partial_{x}^{2}\right)\left(\mathrm{I}-\partial_{x}^{2}\right) u=\frac{1}{2} \int_{x_{\min }}^{x_{\max }}\left(4 u^{2}+5\left(\partial_{x} u\right)^{2}+\left(\partial_{x}^{2} u\right)^{2}\right),
\end{aligned}
$$

are invariants of solutions. In the following, we will construct numerical methods that conserve all invariants (4.38).

\subsubsection{Conservative numerical methods}

Using the splitting in (4.37), semidiscretizations

$$
\partial_{t} \boldsymbol{u}=-\left(4 \mathrm{I}-5 D_{2, a}+D_{4, a}\right)^{-1}\left(D_{1}\left(\boldsymbol{u}\left(4 \mathrm{I}-5 D_{2, b}+D_{4, b}\right) \boldsymbol{u}\right)+\left(D_{1} \boldsymbol{u}\right)\left(4 \mathrm{I}-5 D_{2, b}+D_{4, b}\right) \boldsymbol{u}\right)
$$

that conserve the linear and quadratic invariant can be obtained.

Theorem 4.11. If $D_{1}$ is a periodic first-derivative SBP operator with diagonal mass matrix $M, D_{2, a} \mathcal{E} D_{2, b}$ are periodic second-derivative $S B P$ operators, and $D_{4, a} \mathcal{E} D_{4, b}$ are periodic fourth-derivative $S B P$ operators, then the semidiscretization (4.39) conserves the quadratic invariant (4.38c). If $D_{1}$ commutes with $D_{2, b} \mathcal{E}$ $D_{4, b}$, the linear invariants (4.38a) and (4.38b) are also conserved.

Proof. Because of Lemma 2.27, (4.38a) is conserved if and only if (4.38b) is conserved. For (4.38b), consider

$$
\begin{aligned}
& \frac{\mathrm{d}}{\mathrm{d} t} J_{1}^{\mathrm{HH}}(\boldsymbol{u})=\frac{\mathrm{d}}{\mathrm{d} t} \mathbf{1}^{T} M\left(4 \mathrm{I}-5 D_{2, a}+D_{4, a}\right) \boldsymbol{u}=\mathbf{1}^{T} M\left(4 \mathrm{I}-5 D_{2, a}+D_{4, a}\right) \partial_{t} \boldsymbol{u} \\
= & -\mathbf{1}^{T} M\left(D_{1}\left(\boldsymbol{u} D_{4, b} \boldsymbol{u}\right)+\left(D_{1} \boldsymbol{u}\right)\left(D_{4, b} \boldsymbol{u}\right)-5 D_{1}\left(\boldsymbol{u} D_{2, b} \boldsymbol{u}\right)-5\left(D_{1} \boldsymbol{u}\right)\left(D_{2, b} \boldsymbol{u}\right)+4 D_{1} \boldsymbol{u}^{2}+4 \boldsymbol{u} D_{1} \boldsymbol{u}\right) \\
= & -\mathbf{1}^{T} M\left(\left(D_{1} \boldsymbol{u}\right)\left(D_{4, b} \boldsymbol{u}\right)-5\left(D_{1} \boldsymbol{u}\right)\left(D_{2, b} \boldsymbol{u}\right)\right),
\end{aligned}
$$

where Lemma 2.28 and Lemma 2.27 have been used. If $D_{1}$ commutes with $D_{2, b}$ and $D_{4, b}$,

$$
\frac{\mathrm{d}}{\mathrm{d} t} J_{1}^{\mathrm{HH}}(\boldsymbol{u})=-\boldsymbol{u}^{T} D_{1}^{T} M D_{4} \boldsymbol{u}+5 \boldsymbol{u}^{T} D_{1}^{T} M D_{2} \boldsymbol{u}=0 .
$$

Since $4 \mathrm{I}-5 D_{2, a}+D_{4, a}$ is a symmetric operator, the semidiscrete rate of change of the quadratic invariant $(4.38 \mathrm{c})$ is

$$
\begin{aligned}
\frac{\mathrm{d}}{\mathrm{d} t} J_{2}^{\mathrm{HH}}(\boldsymbol{u})=\frac{1}{2} \frac{\mathrm{d}}{\mathrm{d} t} \boldsymbol{u}^{T} M\left(4 \mathrm{I}-5 D_{2, a}+D_{4, a}\right) \boldsymbol{u}=\boldsymbol{u}^{T} M\left(4 \mathrm{I}-5 D_{2, a}+D_{4, a}\right) \partial_{t} \boldsymbol{u} \\
\quad=-\boldsymbol{u}^{T} M\left(D_{1}\left(\boldsymbol{u}\left(4 \mathrm{I}-5 D_{2, b}+D_{4, b}\right) \boldsymbol{u}\right)+\left(D_{1} \boldsymbol{u}\right)\left(4 \mathrm{I}-5 D_{2, b}+D_{4, b}\right) \boldsymbol{u}\right)=0 .
\end{aligned}
$$

A smooth traveling wave solution with speed $c=1.2$ computed numerically using the Petviashvili method in the periodic domain $[-40,40]$ was used to verify the conservation properties of the semidiscretization (4.39). This traveling wave solution has been computed for the PDE

$$
\left(4-5 \partial_{x}^{2}+\partial_{x}^{4}\right) \partial_{t} u+8 \kappa \partial_{x} u+u \partial_{x}^{5} u+2\left(\partial_{x} u\right) \partial_{x}^{4} u-5 u \partial_{x}^{3} u-10\left(\partial_{x} u\right) \partial_{x}^{2} u+12 u \partial_{x} u=0
$$

which can be transformed to a solution of the $\mathrm{HH}$ equation (with $\kappa=0$ ) by the transformation

$$
x \rightarrow x+\kappa t, \quad u \rightarrow u+\kappa .
$$




\subsubsection{Convergence study in space}

For the following convergence study, the method of manufactured solutions is applied to (4.8) with periodic boundary conditions and the semidiscretization (4.39) with $D_{2, a}=D_{2, b}=D_{2}$ and $D_{4, a}=D_{4, b}=D_{2}^{2}$. The results are shown in Figure 6 .

Similarly to the $\mathrm{BBM}$ and $\mathrm{CH}$ equations, central finite difference methods with order of accuracy $p$ result in an $\mathrm{EOC} \approx p$. Wide-stencil operators $D_{2}=D_{1}^{2}$ for $p \in\{6,8\}$ show a strong dependence of the final error on the parity of the number of nodes. Again, the error is up to an order of magnitude smaller for odd $N$. For $p \in\{2,4\}$ or narrow-stencil operators $D_{2}$, such a dependence is not visible for this test case.

As for the BBM equation and in contrast to the FW, $\mathrm{CH}$, and DP equations, nodal continuous Galerkin methods using Lobatto-Legendre bases with wide stencil operator $D_{2}=D_{1}^{2}$ yield EOC $\approx$ $p+1$ for odd polynomial degrees $p$ and EOC $\approx p$ for even $p$. The error is approximately an order of magnitude smaller for $p=2$ than for $p=1$ for the same number of elements $N$. In contrast, $p=3$ can result in a similar error as $p=4$ for odd numbers of elements $N$ while the error is up to an order of magnitude bigger for even $N$.

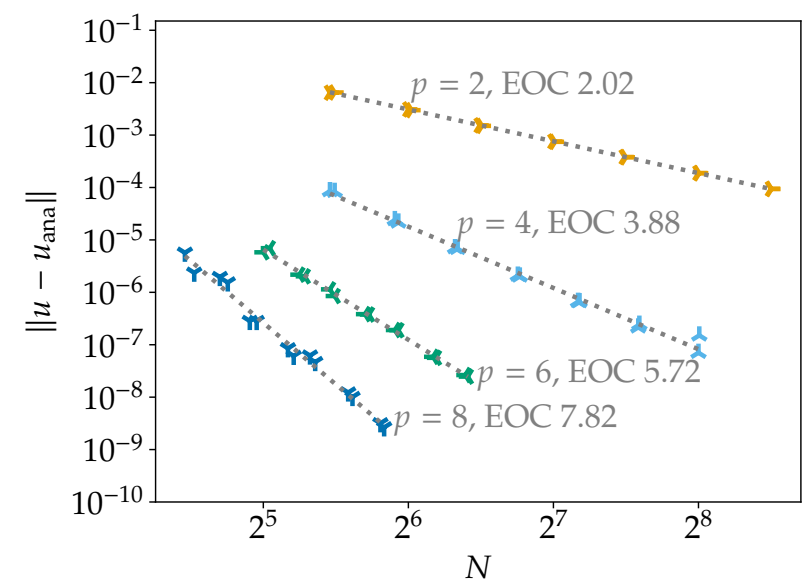

(a) Finite difference methods, narrow stencil $D_{2}$.

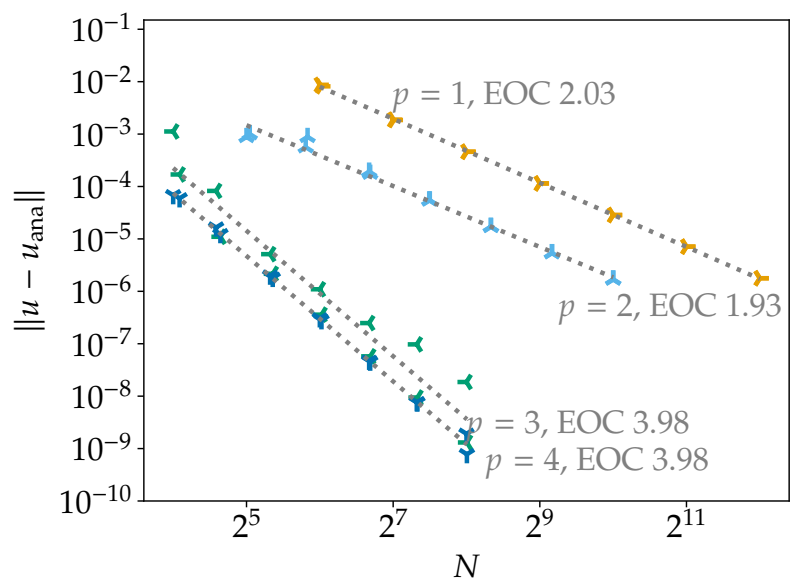

(b) Continuous Galerkin methods, $D_{2}=D_{1}^{2}$.

Figure 6: Convergence results of the spatial semidiscretizations (4.39) with $D_{4}=D_{2}^{2}$ for the manufactured solution (4.8) of the HH equation. All of these semidiscretizations conserve the linear and quadratic invariants (4.38) of the HH equation (4.36).

\subsection{BBM-BBM system}

Consider the system $[6,7,15,18]$

$$
\begin{array}{rlrl}
\partial_{t} \eta(t, x)+\partial_{x} u(t, x)+\partial_{x}(\eta(t, x) u(t, x))-\partial_{t} \partial_{x}^{2} \eta(t, x) & =0, & & t \in(0, T), x \in\left(x_{\min }, x_{\max }\right), \\
\partial_{t} u(t, x)+\partial_{x} \eta(t, x)+\partial_{x} \frac{u(t, x)^{2}}{2}-\partial_{t} \partial_{x}^{2} u(t, x) & =0, & & t \in(0, T), x \in\left(x_{\min }, x_{\max }\right), \\
\eta(0, x) & =\eta^{0}(x), & x \in\left[x_{\min }, x_{\max }\right], \\
u(0, x) & =u^{0}(x), & x \in\left[x_{\min }, x_{\max }\right],
\end{array}
$$

with periodic boundary conditions, which can also be written as

$$
\begin{array}{r}
\partial_{t} \eta(t, x)+\left(\mathrm{I}-\partial_{x, P}^{2}\right)^{-1} \partial_{x}(u(t, x)+\eta(t, x) u(t, x))=0 \\
\partial_{t} u(t, x)+\left(\mathrm{I}-\partial_{x, P}^{2}\right)^{-1} \partial_{x}\left(\eta(t, x)+\frac{u(t, x)^{2}}{2}\right)=0,
\end{array}
$$


where $\left(\mathrm{I}-\partial_{x, P}^{2}\right)^{-1}$ is the inverse of the elliptic operator $\mathrm{I}-\partial_{x}^{2}$ with periodic boundary conditions. The functionals

$$
\begin{aligned}
& J_{1}^{\text {BBM-BBM }}(\eta, u)=\int_{x_{\text {min }}}^{x_{\max }} \eta, \\
& J_{2}^{\text {BBM-BBM }_{\text {max }}}(\eta, u)=\int_{x_{\text {min }}}^{x_{\text {max }}} u, \\
& J_{3}^{\text {BBM-BBM }}(\eta, u)=\int_{x_{\text {min }}}^{x_{\text {max }}}\left(\eta^{2}+(1+\eta) u^{2}\right),
\end{aligned}
$$

are invariants of solutions of (4.45).

Interestingly, only integration by parts but no chain or product rule is necessary to prove conservation of the energy $(4.47 \mathrm{c})$. Hence, all three invariants are conserved semidiscretely if periodic SBP operators are employed and the semidiscretization uses the conservative form

$$
\begin{aligned}
\partial_{t} \boldsymbol{\eta}+\left(\mathrm{I}-D_{2}\right)^{-1} D_{1}(\boldsymbol{u}+\boldsymbol{\eta} \boldsymbol{u}) & =\mathbf{0}, \\
\partial_{t} \boldsymbol{u}+\left(\mathrm{I}-D_{2}\right)^{-1} D_{1}\left(\boldsymbol{\eta}+\frac{1}{2} \boldsymbol{u}^{2}\right) & =\mathbf{0} .
\end{aligned}
$$

Theorem 4.12. If $D_{1}$ is a periodic first-derivative $S B P$ operator and $D_{2}$ is a periodic second-derivative $S B P$ operator, then the semidiscretization (4.48) conserves the linear invariants (4.47a) and (4.47b) of (4.45). If $D_{1} \mathcal{E} D_{2}$ commute, then the quadratic invariant $(4.47 \mathrm{c})$ is also conserved.

Proof. Conservation of the linear invariants $J_{1}^{\mathrm{BBM}-\mathrm{BBM}}, J_{2}^{\mathrm{BBM}-\mathrm{BBM}}$ follows immediately from Lemmas 2.27 and 2.28. Given a matrix $A$, set $\langle\boldsymbol{x}, \boldsymbol{y}\rangle_{A}=\boldsymbol{x}^{T} A \boldsymbol{y}$. Conservation of $J_{3}^{\text {BBM-BBM }}$ follows from Lemmas 2.27 and 2.29, since (letting $L=M\left(\mathrm{I}-D_{2}\right)^{-1} D_{1}$ )

$$
\begin{aligned}
\frac{\mathrm{d}}{\mathrm{d} t} J_{3}^{\mathrm{BBM}-\mathrm{BBM}}(\boldsymbol{\eta}, \boldsymbol{u})= & \frac{\mathrm{d}}{\mathrm{d} t}\left(\langle\boldsymbol{\eta}, \boldsymbol{\eta}\rangle_{M}+\left\langle\boldsymbol{u}^{2}, \boldsymbol{1}+\boldsymbol{\eta}\right\rangle_{M}\right)=\left\langle\partial_{t} \boldsymbol{\eta}, 2 \boldsymbol{\eta}+\boldsymbol{u}^{2}\right\rangle_{M}+\left\langle\partial_{t} \boldsymbol{u}, 2(1+\boldsymbol{\eta}) \boldsymbol{u}\right\rangle_{M} \\
= & -\left\langle\boldsymbol{u}+\boldsymbol{\eta} \boldsymbol{u}, 2 \boldsymbol{\eta}+\boldsymbol{u}^{2}\right\rangle_{L}-2\left\langle\boldsymbol{\eta}+\frac{1}{2} \boldsymbol{u}^{2},(1+\boldsymbol{\eta}) \boldsymbol{u}\right\rangle_{L} \\
= & -2\langle\boldsymbol{u}, \boldsymbol{\eta}\rangle_{L}-2\langle\boldsymbol{\eta} \boldsymbol{u}, \boldsymbol{\eta}\rangle_{L}-\left\langle\boldsymbol{u}, \boldsymbol{u}^{2}\right\rangle_{L}-\left\langle\boldsymbol{\eta} \boldsymbol{u}, \boldsymbol{u}^{2}\right\rangle_{L}-2\langle\boldsymbol{\eta}, \boldsymbol{u}\rangle_{L}-\left\langle\boldsymbol{u}^{2}, \boldsymbol{u}\right\rangle_{L} \\
& -2\langle\boldsymbol{\eta}, \boldsymbol{\eta} \boldsymbol{u}\rangle_{L}-\left\langle\boldsymbol{u}^{2}, \boldsymbol{\eta} \boldsymbol{u}\right\rangle_{L}=0,
\end{aligned}
$$

because $L$ is skew-symmetric by Lemma 2.29.

\section{Reflecting boundary conditions}

Another interesting set of boundary conditions is given by reflecting boundary conditions, i.e.

$$
\begin{array}{rlrl}
\partial_{t} \eta(t, x)+\partial_{x} u(t, x)+\partial_{x}(\eta(t, x) u(t, x))-\partial_{t} \partial_{x}^{2} \eta(t, x) & =0, & & t \in(0, T), x \in\left(x_{\min }, x_{\max }\right), \\
\partial_{t} u(t, x)+\partial_{x} \eta(t, x)+\partial_{x} \frac{u(t, x)^{2}}{2}-\partial_{t} \partial_{x}^{2} u(t, x) & =0, & & t \in(0, T), x \in\left(x_{\min }, x_{\max }\right), \\
\partial_{x} \eta(t, x) & =0, & & t \in(0, T), x \in\left\{x_{\min }, x_{\max }\right\}, \\
u(t, x) & =0, & & t \in(0, T), x \in\left\{x_{\min }, x_{\max }\right\}, \\
\eta(0, x) & =\eta^{0}(x), & x \in\left[x_{\min }, x_{\max }\right], \\
u(0, x) & =u^{0}(x), & x \in\left[x_{\min }, x_{\max }\right] .
\end{array}
$$

Such boundary conditions occur in the study of two-way dispersive waves in fluids subject to solid wall boundary conditions [6]. For this system, the total mass of $\eta$ (4.47a) and the total energy $(4.47 \mathrm{c})$ are still conserved, but the total mass of $u(4.47 \mathrm{~b})$ is not necessarily conserved. 
To create a conservative semidiscretization, the following operators will be used. The projection operator $P_{D}=\operatorname{diag}(0,1, \ldots, 1,0)$ maps onto the space of grid functions with homogeneous Dirichlet boundary conditions. For an SBP first-derivative operator $D_{1}$, denote the second derivative operator induced by $\mathrm{I}-D_{1}^{2}$

- with strong imposition of homogeneous Dirichlet boundary conditions as $I-D_{2, D}$. The corresponding solution operator satisfies

$$
\boldsymbol{v}=\left(\mathrm{I}-D_{2, D}\right)^{-1} \boldsymbol{w} \Longleftrightarrow P_{D}\left(\mathrm{I}-D_{2, D}\right) \boldsymbol{v}=P_{D} \boldsymbol{w} \wedge\left(\mathrm{I}-P_{D}\right) \boldsymbol{v}=\mathbf{0} .
$$

- with weak-strong imposition of homogeneous Neumann boundary conditions as $\mathrm{I}-D_{2, N}$. The corresponding solution operator satisfies

$$
\boldsymbol{v}=\left(\mathrm{I}-D_{2, N}\right)^{-1} \boldsymbol{w} \Longleftrightarrow\left(\mathrm{I}+M^{-1} D_{1}^{T} M P_{D} D_{1}\right) \boldsymbol{v}=\boldsymbol{w} .
$$

This discretization uses neither a strong imposition of boundary conditions (setting $\boldsymbol{e}_{L / R}^{T} D_{1}\left(\mathrm{I}-D_{2, N}\right)^{-1}=$ $0^{T}$ and solving the PDE in the interior) nor the usual weak imposition (where (4.51) is used without the additional projection $P_{D}$ ).

These operators correspond to I $-D_{1}^{2}$ in the interior and are modified near the boundaries to impose the boundary conditions. Using these operators results in the semidiscretization

$$
\begin{aligned}
\partial_{t} \boldsymbol{\eta}+\left(\mathrm{I}-D_{2, N}\right)^{-1} D_{1}(\boldsymbol{u}+\boldsymbol{\eta} \boldsymbol{u}) & =\mathbf{0}, \\
\partial_{t} \boldsymbol{u}+\left(\mathrm{I}-D_{2, D}\right)^{-1} D_{1}\left(\boldsymbol{\eta}+\frac{1}{2} \boldsymbol{u}^{2}\right) & =0 .
\end{aligned}
$$

Theorem 4.13. If $D_{1}$ is a first-derivative SBP operator and the initial condition for $\boldsymbol{u}$ satisfies the homogeneous Dirichlet boundary condition, then the semidiscretization (4.52) conserves the two invariants of (4.49), i.e. the total mass of $\eta$ (4.47a) and the total energy (4.47c).

Proof. The semidiscretization (4.52) can be written as

$$
\begin{aligned}
\left(\mathrm{I}-D_{2, N}\right) \partial_{t} \boldsymbol{\eta}+D_{1}(\boldsymbol{u}+\boldsymbol{\eta} \boldsymbol{u}) & =\mathbf{0}, \\
\left(\mathrm{I}-D_{2, D}\right) \partial_{t} \boldsymbol{u}+D_{1}\left(\boldsymbol{\eta}+\frac{1}{2} \boldsymbol{u}^{2}\right) & =\mathbf{0} .
\end{aligned}
$$

Since $\boldsymbol{e}_{L / R}^{T} \partial_{t} \boldsymbol{u}=0$, the homogeneous Dirichlet boundary condition for $\boldsymbol{u}$ is satisfied for all times if it is satisfied initially.

The total mass of $\eta$ is conserved, since the strong imposition of homogeneous Dirichlet boundary conditions for $u$ yields

$$
\begin{aligned}
\mathbf{1}^{T} M \partial_{t} \boldsymbol{\eta} & =-\mathbf{1}^{T} M D_{1}(\boldsymbol{u}+\boldsymbol{\eta} \boldsymbol{u})+\mathbf{1}^{T} M D_{2, N} \partial_{t} \boldsymbol{\eta} \\
& =-\mathbf{1}^{T} M D_{1}(\boldsymbol{u}+\boldsymbol{\eta} \boldsymbol{u})-\mathbf{1}^{T} D_{1}^{T} M P_{D} D_{1} \partial_{t} \boldsymbol{\eta} \\
& =-\mathbf{1}^{T}\left(\boldsymbol{e}_{R} \boldsymbol{e}_{R}^{T}-\boldsymbol{e}_{L} \boldsymbol{e}_{L}^{T}\right)(\boldsymbol{u}+\boldsymbol{\eta} \boldsymbol{u})=0 .
\end{aligned}
$$

To compute the semidiscrete rate of change of the energy, observe that

$$
\left(-D_{1} \partial_{t} \boldsymbol{u}\right)^{T} M \partial_{t} \boldsymbol{\eta}+\left(-D_{1} \partial_{t} \boldsymbol{\eta}\right)^{T} M \partial_{t} \boldsymbol{u}=0 .
$$

Hence,

$$
\begin{aligned}
\frac{\mathrm{d}}{\mathrm{d} t} J_{3}^{\text {BBM-BBM }}(\boldsymbol{\eta}, \boldsymbol{u}) & =\left(2 \boldsymbol{\eta}+\boldsymbol{u}^{2}\right)^{T} M \partial_{t} \boldsymbol{\eta}+2(\boldsymbol{u}+\boldsymbol{\eta} \boldsymbol{u})^{T} M \partial_{t} \boldsymbol{u} \\
& =\left(2 \boldsymbol{\eta}+\boldsymbol{u}^{2}-2 D_{1} \partial_{t} \boldsymbol{u}\right)^{T} M \partial_{t} \boldsymbol{\eta}+2\left(\boldsymbol{u}+\boldsymbol{\eta} \boldsymbol{u}-2 D_{1} \partial_{t} \boldsymbol{\eta}\right)^{T} M \partial_{t} \boldsymbol{u} \\
& =\left(2 \boldsymbol{\eta}+\boldsymbol{u}^{2}-2 D_{1} \partial_{t} \boldsymbol{u}\right)^{T} M \partial_{t} \boldsymbol{\eta}+2\left(\boldsymbol{u}+\boldsymbol{\eta} \boldsymbol{u}-2 D_{1} \partial_{t} \boldsymbol{\eta}\right)^{T} P_{D}^{T} M \partial_{t} \boldsymbol{u},
\end{aligned}
$$


because of the strong imposition of the homogeneous Dirichlet boundary conditions for $\partial_{t} \boldsymbol{u}$. Inserting the semidiscretization (4.53) and using again the homogeneous Dirichlet boundary conditions for $\boldsymbol{u}$ results in

$$
\begin{aligned}
\frac{\mathrm{d}}{\mathrm{d} t} J_{3}^{\text {BBM-BBM }}(\boldsymbol{\eta}, \boldsymbol{u})= & -\left(2 \boldsymbol{\eta}+\boldsymbol{u}^{2}-2 D_{1} \partial_{t} \boldsymbol{u}\right)^{T} M\left(D_{1}(\boldsymbol{u}+\boldsymbol{\eta} \boldsymbol{u})-D_{2, N} \partial_{t} \boldsymbol{\eta}\right) \\
& -\left(\boldsymbol{u}+\boldsymbol{\eta} \boldsymbol{u}-2 D_{1} \partial_{t} \boldsymbol{\eta}\right)^{T} P_{D}^{T} M\left(D_{1}\left(2 \boldsymbol{\eta}+\boldsymbol{u}^{2}\right)-D_{2, D} \partial_{t} \boldsymbol{u}\right) \\
= & +\left(2 \boldsymbol{\eta}+\boldsymbol{u}^{2}-2 D_{1} \partial_{t} \boldsymbol{u}\right)^{T} D_{1}^{T} M P_{D}\left((\boldsymbol{u}+\boldsymbol{\eta} \boldsymbol{u})+D_{1} \partial_{t} \boldsymbol{\eta}\right) \\
& -\left(\boldsymbol{u}+\boldsymbol{\eta} \boldsymbol{u}-2 D_{1} \partial_{t} \boldsymbol{\eta}\right)^{T} P_{D}^{T} M D_{1}\left(\left(2 \boldsymbol{\eta}+\boldsymbol{u}^{2}\right)-D_{1} \partial_{t} \boldsymbol{u}\right)=0 .
\end{aligned}
$$

Remark 4.14. A smooth traveling wave solution with speed $c=1.2$ computed numerically using the Petviashvili method in the periodic domain $[-90,90]$ was used to verify the conservation properties of the semidiscretizations (4.48) and (4.52). As expected, relaxation methods in time conserve all linear and the chosen nonlinear invariant up to roundoff errors; cf. Section 4.6.3.

\subsubsection{Convergence study in space}

For the following convergence study, the method of manufactured solutions is applied to

$$
\eta(t, x)=\mathrm{e}^{t} \cos (2 \pi(x-2 t)), \quad u(t, x)=\mathrm{e}^{t / 2} \sin (2 \pi(x-t / 2)),
$$

with periodic boundary conditions in the domain $[0,1]$ for $t \in[0,1]$. The results are shown in Figure 7.

Similarly to the single BBM equation, central finite difference methods with order of accuracy $p$ yield an $\mathrm{EOC} \approx p$. Again, the results for wide-stencil and narrow-stencil second-derivative operators are similar but the narrow-stencil operators result again in smaller errors (up to an order of magnitude).

The results for nodal continuous Galerkin methods using Lobatto-Legendre bases are very similar to the ones for the single BBM equation. Wide-stencil operators $D_{2}=D_{1}^{2}$ yield EOC $\approx p+1$ for odd polynomial degrees $p$ and $\mathrm{EOC} \approx p$ for even $p$. In contrast, the usual narrow-stencil approximation results in an EOC $\approx p+1$ for $p=1$ and an EOC $\approx p+2$ for $p>1$. However, in contrast to the single BBM equation, only the wide-stencil operators yield energy-conservative methods.

A similar observation can be made for nodal discontinuous Galerkin methods. There, widestencil operators $D_{2}=D_{1}^{2}$ yield EOC $\approx p+1$ for even polynomial degrees $p$ and EOC $\approx p$ for odd $p$. The narrow-stencil LDG operator $D_{2}=D_{1,+} D_{1,-}$ results in an EOC $\approx p+1$ for all $p$. Again, only the narrow-stencil operators result in energy-conservative methods.

\section{Reflecting boundary conditions}

A similar procedure is used for reflecting boundary conditions. The method of manufactured solutions is applied to

$$
\eta(t, x)=\mathrm{e}^{2 t} \cos (\pi x), \quad u(t, x)=\mathrm{e}^{t} x \sin (\pi x),
$$

in the domain $[0,1]$ for $t \in[0,1]$. Results of a convergence study for the semidiscretization (4.53) are shown in Figure 8.

The FD methods can be expected to converge with an EOC $\approx p / 2+1$ because of the order of accuracy $p / 2$ near the boundary. However, there is a clear influence of the parity of the number of nodes $N$ for some operators: For $p=6$, odd $N$ yield more than an order of magnitude smaller errors and a slightly bigger EOC. For $p=8$, the behavior for even and odd $N$ is the other way round. For $p=4$, there is no significant influence. In particular, it is noteworthy that using one node less or more can result in an increase or decrease of the order of magnitude of the error. 


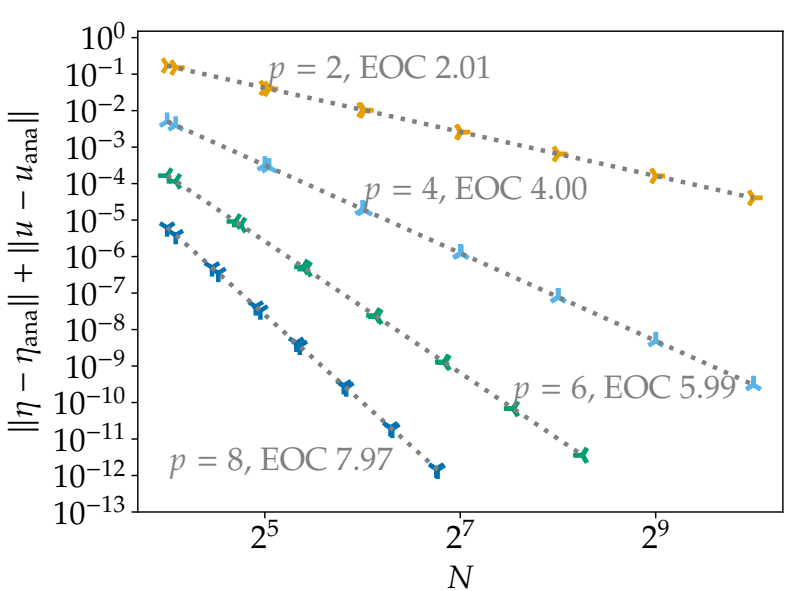

(a) Finite difference methods, wide stencil $D_{2}=D_{1}^{2}$.

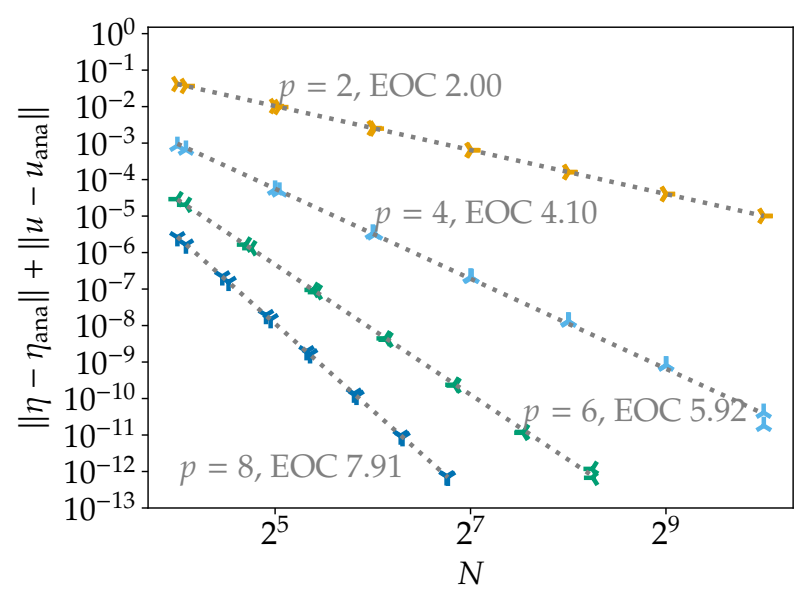

(b) Finite difference methods, narrow stencil $D_{2}$.

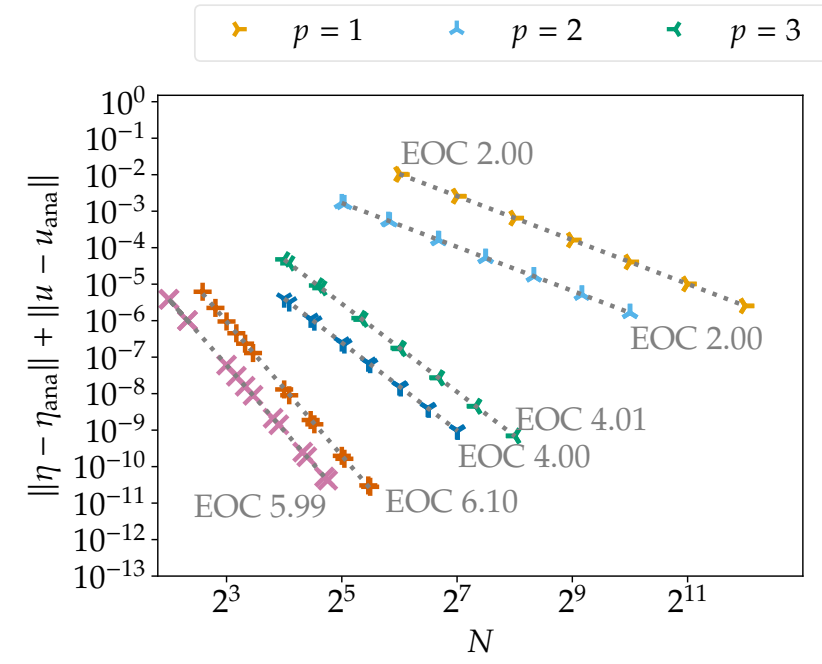

ฯ $p=4+p=5 \quad$ × $p=6$

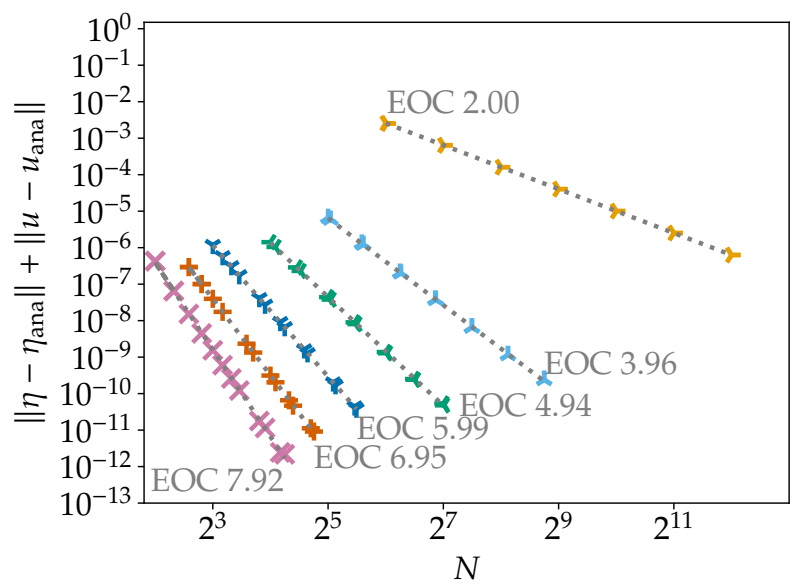

(c) Continuous Galerkin methods, $D_{2}=D_{1}^{2}$.

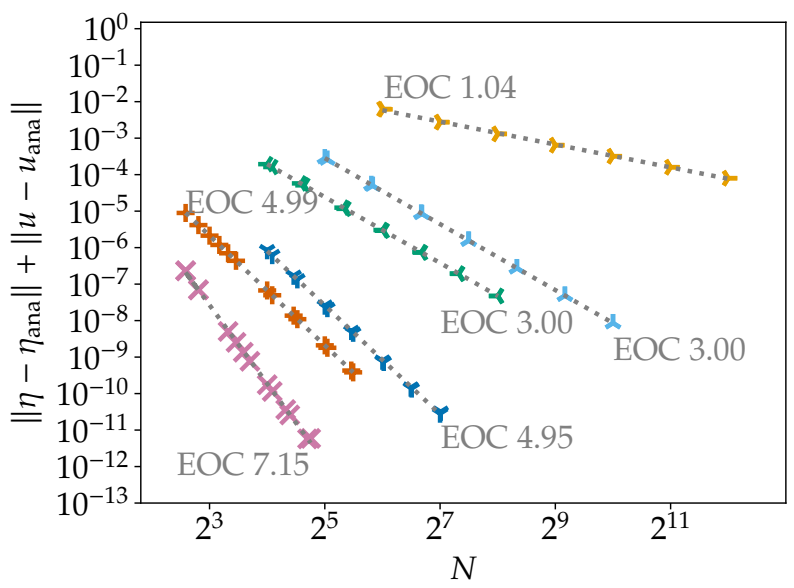

(d) Continuous Galerkin methods, narrow stencil $D_{2}$.

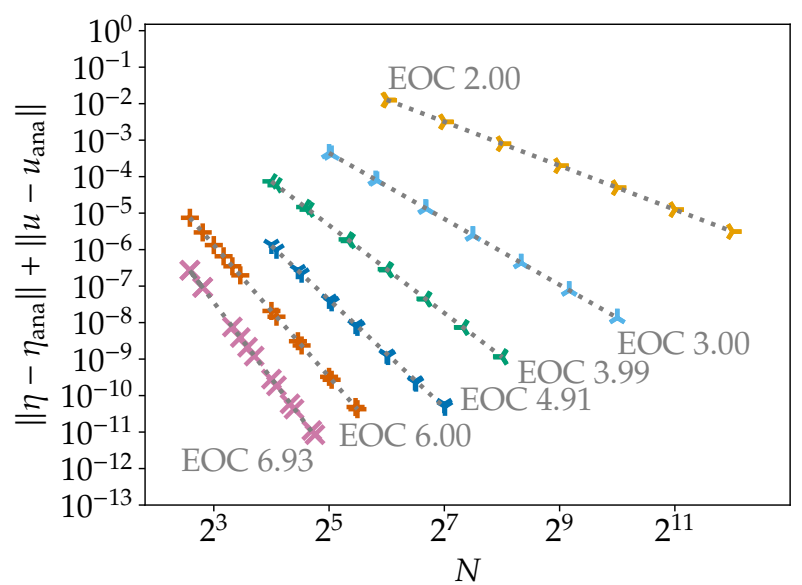

(e) Discontinuous Galerkin methods, $D_{2}=D_{1}^{2}$.

(f) Discontinuous Galerkin methods, $D_{2}=D_{1,+} D_{1,-}$.

Figure 7: Convergence results of the spatial semidiscretizations (4.48) for the manufactured solution (4.58) of the BBM-BBM system. All of these semidiscretizations conserve the linear invariants (4.47) of the BBM-BBM system (4.45). The FD methods and the Galerkin methods with wide stencil $D_{2}$ conserve the quadratic invariant as well. 


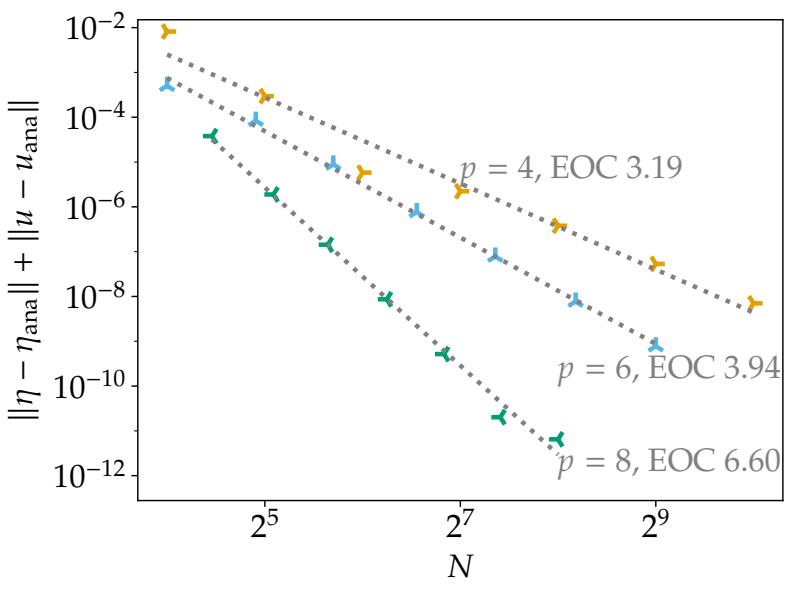

(a) Accurate FD operators of [68], even N.

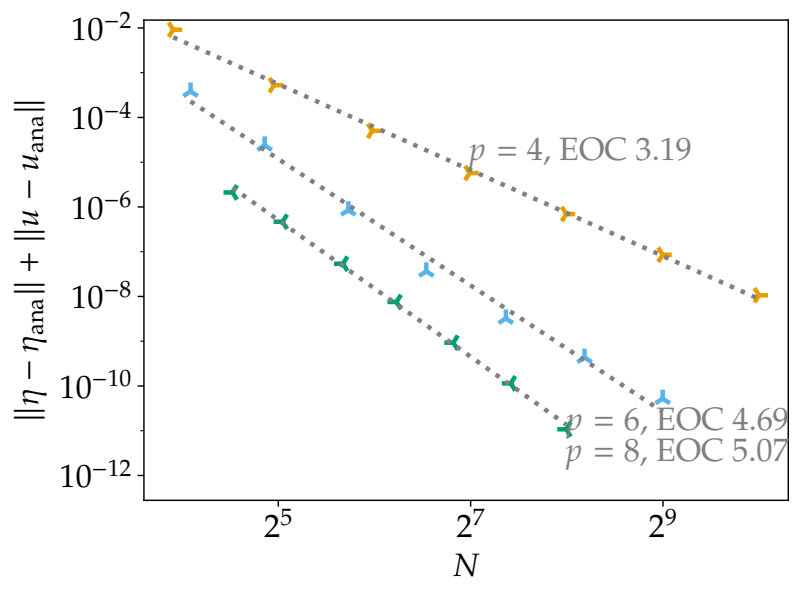

(b) Accurate FD operators of [68], odd N.

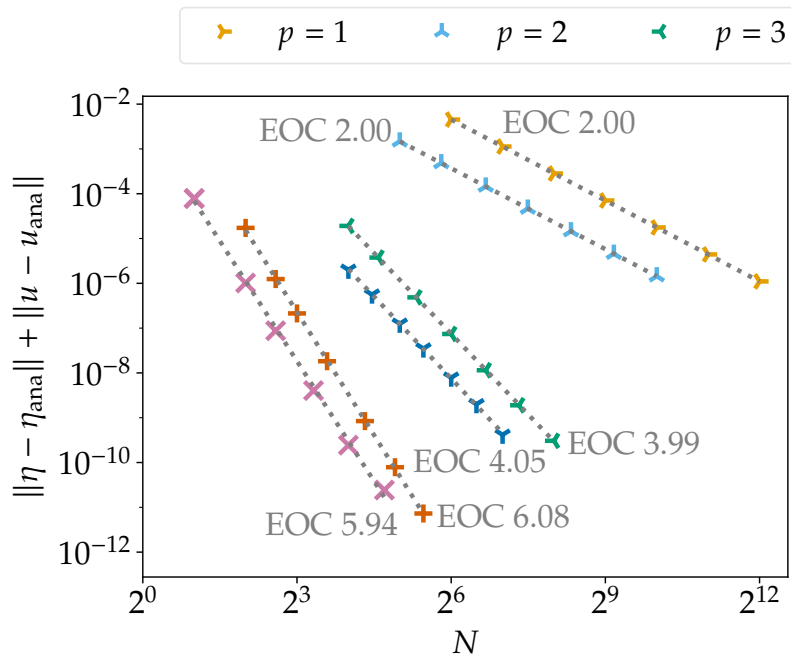

(c) Continuous Galerkin methods, even $N$.

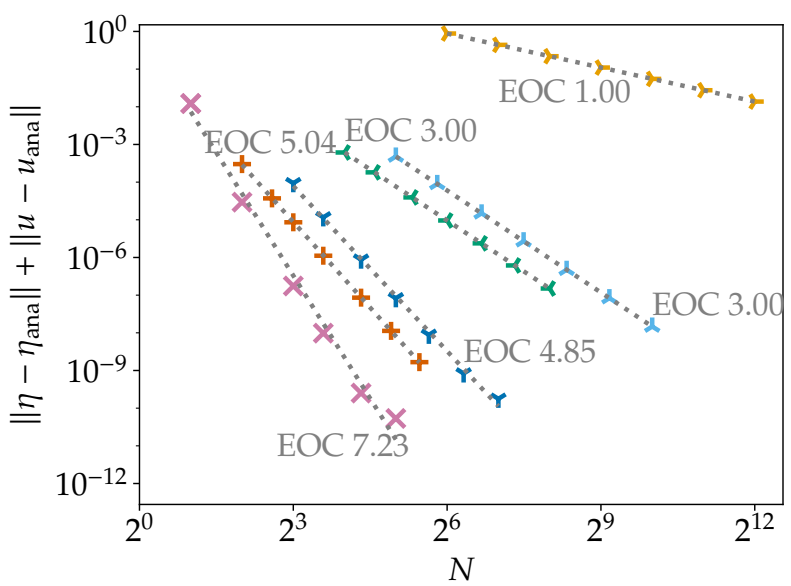

(e) Discontinuous Galerkin methods, even N.

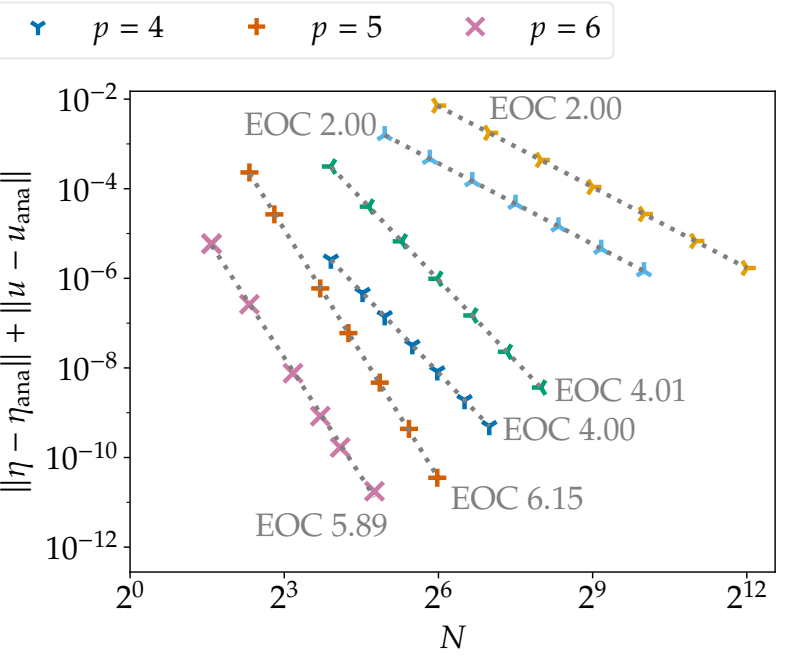

(d) Continuous Galerkin methods, odd N.

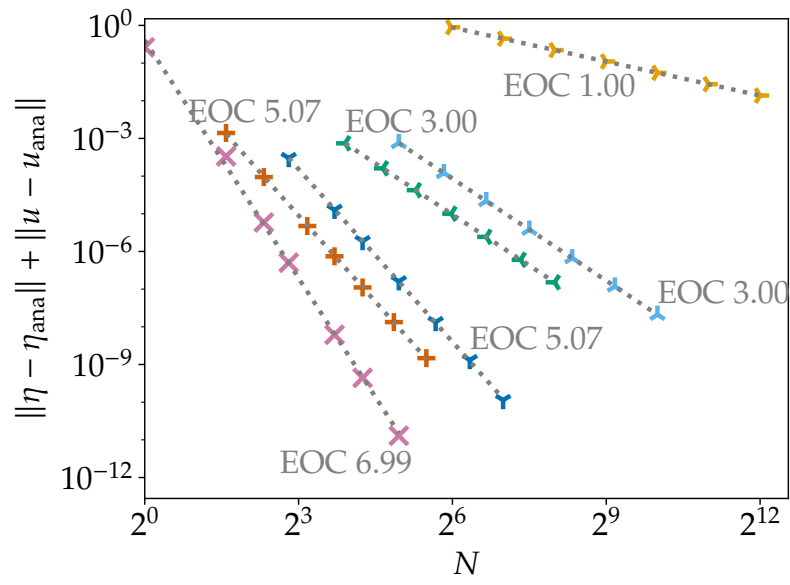

(f) Discontinuous Galerkin methods, odd N.

Figure 8: Convergence results of the spatial semidiscretizations (4.53) for the manufactured solution (4.59) of the BBM-BBM equation (4.49) with reflecting boundary conditions. 
For continuous Galerkin methods with wide-stencil operator $D_{2}=D_{1}^{2}$, EOC $\approx p+1$ for odd $p$ and $\mathrm{EOC} \approx p$ for even $p$ as for periodic $\mathrm{BCs}$. There is a significant influence of the parity of the number of elements $N$ for the odd polynomial degrees $p \in\{3,5\}$. An even number of elements can reduce the error by an order of magnitude.

Finally, discontinuous Galerkin methods reproduce the EOC $\approx p+1$ for even $p$ and EOC $\approx p$ for odd $p$ as for periodic BCs and wide-stencil second-derivative operators. The influence of the parity of the number of elements is much less pronounced than for CG methods.

\subsubsection{Convergence study for long-time simulations of traveling waves}

For long time simulations, structure-preserving methods such as the conservative semidiscretizations (4.48) with wide-stencil operators $D_{2}=D_{1}^{2}$ coupled with relaxation methods in time can yield both qualitative and quantitative improvements over standard methods. To demonstrate this, we consider the traveling wave solutions described in Remark 4.14 with a final time $t=7500$, corresponding to 50 periods.

In the following, CG methods with $p=4$ and DG methods with $p=3$ are used. The sixthorder accurate Runge-Kutta method of [98] is used with a time step $\Delta t \propto \Delta x$ for a space-time convergence study. The standard schemes use the narrow-stencil second-derivative operators and the baseline time integration method. For the conservative schemes, the wide-stencil secondderivative operators $D_{2}=D_{1}^{2}$ are combined with relaxation in time.

As can be seen in Figure 9, the EOC of the standard methods is higher but the absolute error of the conservative methods is still lower for the applied range of parameters. Increasing the resolution even further, it can be expected that the error will be smaller for the standard methods. On the other hand, the conservative methods will probably still result in a smaller error for even longer simulation times. Moreover, they are more efficient at reasonably small error tolerances.

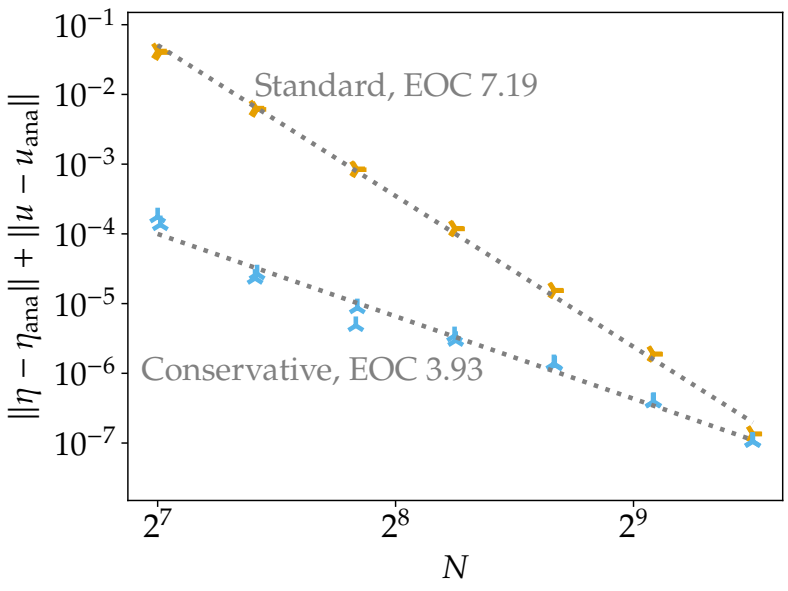

(a) Continuous Galerkin methods, $p=4$.

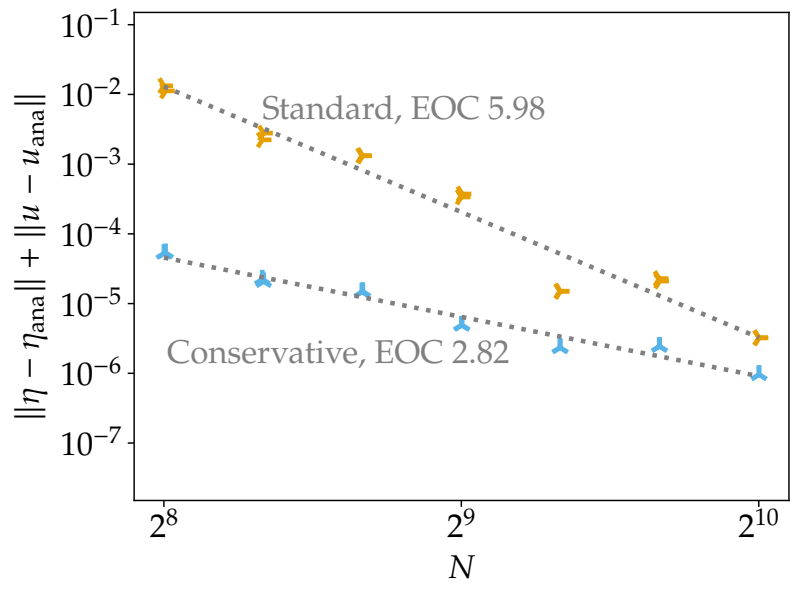

(b) Discontinuous Galerkin methods, $p=3$.

Figure 9: Convergence results of the spatial semidiscretizations (4.48) for a long time simulation of a traveling wave solution of the BBM-BBM equation (4.45).

\subsubsection{Conservation of invariants for reflecting boundary conditions}

To test the method for reflecting boundary conditions, the traveling wave initial condition described in Remark 4.14 is used. For SBP finite difference methods with interior order of accuracy $p=6$, results are visualized in Figure 10. The classical operators of [69] result in undesired oscillations of small amplitude at the final time $t=3050$ which vanish under grid refinement. These are generated by the interaction of the wave with the boundary and can also be reduced significantly by applying operators with improved accuracy near the boundary, e.g. the ones of [67] or [68]. 
Again, applying relaxation to conserve the energy improves the accuracy of the fully-discrete methods. Choosing $\Delta t=0.1$ for the accurate operators of [68], the solutions with and without relaxation are visually indistinguishable and further refinement causes no visible change. Increasing the time step by a factor of ten, the solution of the baseline method is clearly distorted with changes of the amplitude and phase while the relaxation solution is barely affected.

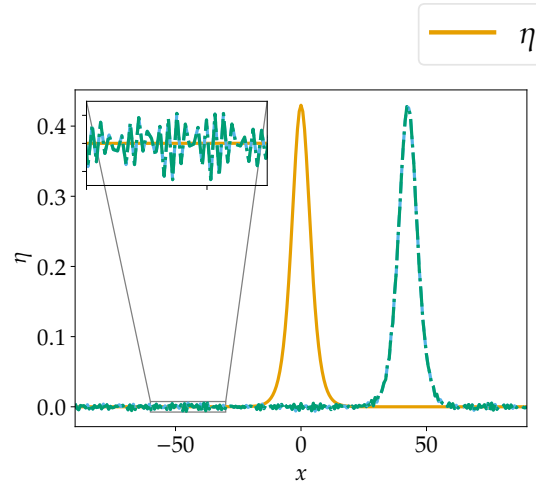

(a) Classical operator of [69], $\Delta t=0.1$.

$\eta^{0} \quad---\eta$ (baseline) $\quad-\cdot-\eta$ (relaxation)

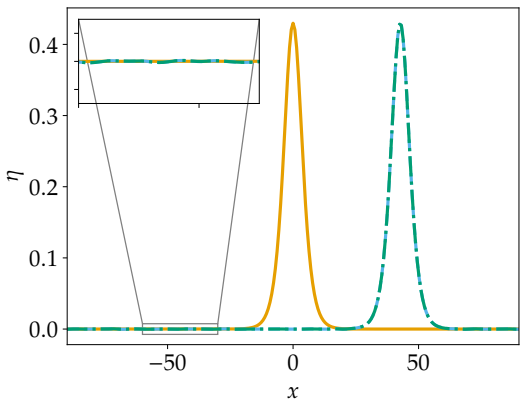

(b) Accurate operator of [68], $\Delta t=0.1$

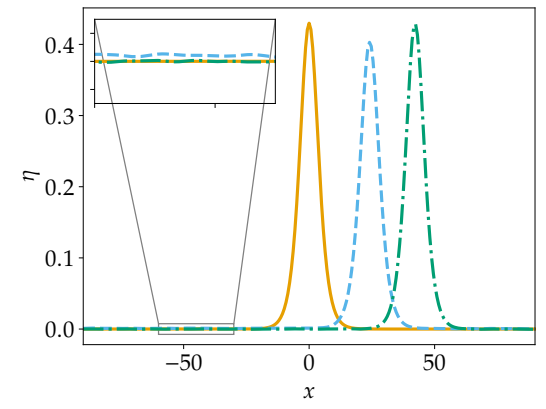

(c) Accurate operator of [68], $\Delta t=1.0$.

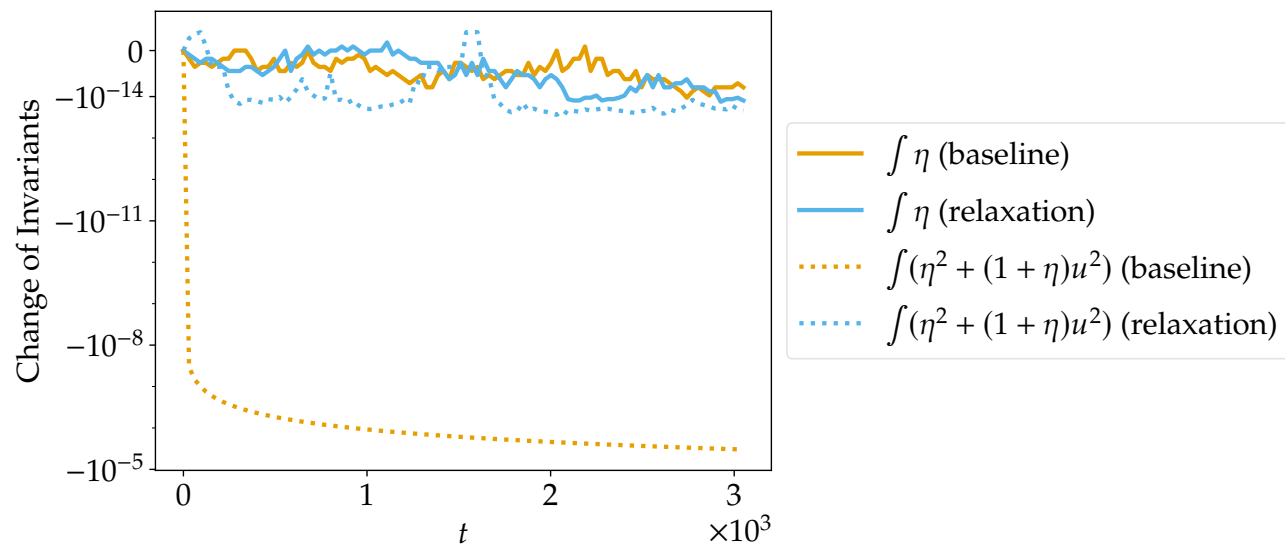

(d) Change of the invariants (4.47) for the accurate operator of [68] with $\Delta t=0.1$.

Figure 10: Numerical solutions of the BBM-BBM system with reflecting boundary conditions (4.49) obtained by finite difference methods and RK4 with and without relaxation to preserve the energy (4.47c).

\section{Summary and conclusions}

We have further developed general tools to construct conservative methods and applied them to a broad range of dispersive wave equation models. These fully-discrete schemes combine summation by parts operators in space with relaxation methods in time to conserve all linear and one nonlinear invariant for each model. Because of the generality of the SBP framework, the conservation properties of four different classes of schemes, namely Fourier collocation, finite difference, continuous Galerkin, and discontinuous Galerkin methods, can be analyzed simultaneously for six different dispersive wave models studied in this article. Instead of requiring $4 \cdot 6=24$ ad hoc approaches, we have established general results first, allowing a unified analysis and relatively simple proofs of conservation for all methods. The proposed schemes do not require exact integration (as long as the mass matrix is diagonal) and use time discretizations that are explicit except for the solution of a scalar equation at each step.

While the application of the relaxation approach is straightforward given the established results, the construction of conservative spatial semidiscretizations requires some tuning for each model. 
Nevertheless, the only required techniques are the application of split forms and the special choice of higher-derivative operators. In bounded domains, the carefully developed imposition of boundary conditions is also crucial.

Having developed a broad framework of conservative numerical methods for dispersive wave equations, the present work will be extended in the future. While the analysis of conservation properties for different classes of methods can be conducted in the unifying SBP framework, the detailed study of error estimates still seems to require specializations on the schemes and does not fit into this manuscript. Estimates of the order of convergence resulting from numerical experiments are summarized in Table 1.

As alluded to in the introduction, conservative fully-discrete numerical methods can have improved properties concerning the error growth in time. This is related to the results shown in Section 4.6.2, where standard numerical methods can have a higher order of convergence but result still in bigger errors than conservative numerical methods. We will focus on these aspects in the future.

Table 1: Summary of the experimental order of convergence (EOC) and conservation properties (Cons.) for various semidiscretizations of the dispersive wave equations considered in this manuscript. The methods conserve either the linear invariant(s) only (lin.), a chosen nonlinear invariant only (nonl.), or both. For FD methods, $p$ is the interior order of accuracy. For CG and DG methods, $p$ is the polynomial degree. If no EOC is given, the results of numerical experiments were not clear enough.

\begin{tabular}{|c|c|c|c|c|c|c|c|c|c|}
\hline \multirow{2}{*}{ Class } & \multirow{2}{*}{$\begin{array}{r}\text { Method } \\
D_{2} \text { stencil }\end{array}$} & \multirow[b]{2}{*}{$p$} & \multicolumn{2}{|c|}{ BBM (4.5) } & \multicolumn{3}{|c|}{ FW (4.13) } & \multicolumn{2}{|c|}{$\mathrm{CH}(4.20), \alpha=1 / 2$} \\
\hline & & & Cons. & EOC & Cons. & EOC & & Cons. & $\mathrm{EOC}$ \\
\hline FD & & & both & $\approx p$ & both & $\in[p-$ & $/ 2, p]$ & both & $\approx p$ \\
\hline \multirow[t]{4}{*}{ CG } & \multirow[t]{2}{*}{ wide } & \multirow{4}{*}{$\begin{array}{l}\text { odd } p \\
\text { even } p \\
\text { odd } p \\
\text { even } p\end{array}$} & both & $\approx p+1$ & both & \multirow{4}{*}{\multicolumn{2}{|c|}{$\begin{array}{l}\in[p+1 / 2, p+1] \\
\approx p+1 \\
\in[p+1 / 2, p+1] \\
\approx p+1\end{array}$}} & both & $\approx p+1$ \\
\hline & & & & & & & & & \\
\hline & narrow & & both & $\approx p+2^{\mathrm{a}}$ & lin. & & & both & $\approx p+1$ \\
\hline & & & & $\approx p+2$ & & & & & $\approx p$ \\
\hline \multirow[t]{4}{*}{ DG } & wide & odd $p$ & both & $\approx p$ & both & \multirow{2}{*}{\multicolumn{2}{|c|}{$\begin{array}{l}\approx p \\
\approx p+1\end{array}$}} & both & $\approx p$ \\
\hline & & even $p$ & & $\approx p+1$ & & & & & $\approx p+1$ \\
\hline & narrow & odd $p$ & both & $\approx p+1$ & lin. & \multirow{2}{*}{\multicolumn{2}{|c|}{$\begin{array}{l}\approx p \\
\approx p+1\end{array}$}} & both & $\approx p^{\mathrm{b}}$ \\
\hline & & even $p$ & & $\approx p+1$ & & & & & $\approx p+1$ \\
\hline & \multicolumn{2}{|l|}{ Method } & \multicolumn{3}{|c|}{$\mathrm{DP}(4.29)$} & \multicolumn{2}{|c|}{ c $\mathrm{HH}(4.39)$} & \multicolumn{2}{|c|}{ "BBM-BBM (4.48) } \\
\hline Class & $D_{2}$ stencil & $p$ & Cons. & EOC & & Cons. & EOC & Cons. & EOC \\
\hline FD & & & both & $\in[p-1]$ & & both & $\approx p$ & both & $\approx p$ \\
\hline \multirow[t]{4}{*}{ CG } & \multirow[t]{2}{*}{ wide } & \multirow{4}{*}{$\begin{array}{l}\text { odd } p \\
\text { even } p \\
\text { odd } p \\
\text { even } p\end{array}$} & both & \multirow{4}{*}{\multicolumn{2}{|c|}{$\begin{array}{l}\in[p+1 / 2, p+1] \\
\approx p \\
\in[p+1 / 2, p+1] \\
\approx p\end{array}$}} & both & $\approx p+1$ & both & $\approx p+1$ \\
\hline & & & & & & & $\approx p$ & & $\approx p$ \\
\hline & narrow & & both & & & nonl. & & lin. & $\approx p+2^{\mathrm{a}}$ \\
\hline & & & & & & & & & $\approx p+2$ \\
\hline \multirow[t]{4}{*}{ DG } & wide & odd $p$ & both & $\approx p$ & & both & & both & $\approx p$ \\
\hline & & even $p$ & & $\approx p+1$ & & & & & $\approx p+1$ \\
\hline & narrow & odd $p$ & both & $\approx p$ & & nonl. & & lin. & $\approx p+1$ \\
\hline & & even $p$ & & $\approx p+1$ & & & & & $\approx p+1$ \\
\hline
\end{tabular}

${ }^{\mathrm{a}} p+1=2$ for $p=1$.

${ }^{\mathrm{b}}$ For $p=1$ and $\alpha=1 / 2$, this DG method does not converge for the manufactured solution. However, it converges for other $p$, other $\alpha$ such as $\alpha=1$, and a traveling wave solution. 


\section{Acknowledgments}

Research reported in this publication was supported by the King Abdullah University of Science and Technology (KAUST). DM expresses his gratitude to KAUST for the hospitality and financial support especially during his last visit where he met DK and HR.

\section{References}

[1] R. Abgrall, J. Nordström, P. Öffner, and S. Tokareva. Analysis of the SBP-SAT Stabilization for Finite Element Methods Part I: Linear problems. Dec. 2019. arXiv: 1912.08108 [math. NA].

[2] R. Abgrall, J. Nordström, P. Öffner, and S. Tokareva. Analysis of the SBP-SAT Stabilization for Finite Element Methods Part II: Entropy Stability. Dec. 2019. arXiv: 1912.08390 [math.NA].

[3] G. Alefeld, F. A. Potra, and Y. Shi. "Algorithm 748: Enclosing Zeros of Continuous Functions." In: ACM Transactions on Mathematical Software (TOMS) 21.3 (1995), pp. 327-344. Dor: 10.1145/210089.210111.

[4] D. Antonopoulos, V. Dougalis, and D. Mitsotakis. "Galerkin approximations of periodic solutions of Boussinesq systems." In: Bull. Greek Math. Soc 57.13-30 (2010), p. 1.

[5] D. C. Antonopoulos, V. A. Dougalis, and D. E. Mitsotakis. "Error estimates for Galerkin finite element methods for the Camassa-Holm equation." In: Numerische Mathematik 142.4 (2019), pp. 833-862. DOr: 10.1007/s00211-019-01045-7.

[6] D. C. Antonopoulos, V. A. Dougalis, and D. E. Mitsotakis. "Initial-boundary-value problems for the Bona-Smith family of Boussinesq systems." In: Advances in Differential Equations 14.1/2 (2009), pp. 27-53.

[7] D. C. Antonopoulos, V. A. Dougalis, and D. E. Mitsotakis. "Numerical solution of Boussinesq systems of the Bona-Smith family." In: Applied Numerical Mathematics 60.4 (2010), pp. 314336. Dor: $10.1016 / \mathrm{j}$. apnum.2009.03.002.

[8] A Araújo and A. Durán. "Error propagation in the numerical integration of solitary waves. The regularized long wave equation." In: Applied Numerical Mathematics 36.2-3 (2001), pp. 197-217. Dor: 10.1016/S0168-9274(99)00148-8.

[9] D. N. Arnold, F. Brezzi, B. Cockburn, and L. D. Marini. “Unified analysis of discontinuous Galerkin methods for elliptic problems." In: SIAM Journal on Numerical Analysis 39.5 (2002), pp. 1749-1779. DOI: 10.1137/S0036142901384162.

[10] F. Bassi and S. Rebay. "A high-order accurate discontinuous finite element method for the numerical solution of the compressible Navier-Stokes equations." In: Journal of Computational Physics 131.2 (1997), pp. 267-279. Dor: 10.1006/jcph. 1996. 5572.

[11] T. B. Benjamin, J. L. Bona, and J. J. Mahony. "Model equations for long waves in nonlinear dispersive systems." In: Philosophical Transactions of the Royal Society of London. Series A, Mathematical and Physical Sciences 272.1220 (1972), pp.47-78. Dor: 10. 1098/rsta . 1972.0032.

[12] J. Bezanson, A. Edelman, S. Karpinski, and V. B. Shah. "Julia: A Fresh Approach to Numerical Computing." In: SIAM Review 59.1 (2017), pp. 65-98. Dor: 10 . 1137/141000671. arXiv: 1411.1607 [CS.MS].

[13] J. L. Bona, H. Chen, O. Karakashian, and M. M. Wise. “Finite Element Methods for a System of Dispersive Equations." In: Journal of Scientific Computing 77.3 (2018), pp. 1371-1401. Dor: 10.1007/s10915-018-0767-x.

[14] J. L. Bona and M. Chen. "A Boussinesq system for two-way propagation of nonlinear dispersive waves." In: Physica D: Nonlinear Phenomena 116.1-2 (1998), pp. 191-224. Dor: 10.1016/S0167-2789(97)00249-2. 
[15] J. L. Bona, M. Chen, and J.-C. Saut. "Boussinesq Equations and Other Systems for SmallAmplitude Long Waves in Nonlinear Dispersive Media. I: Derivation and Linear Theory." In: Journal of Nonlinear Science 12.4 (2002). Dor: 10.1007/s00332-002-0466-4.

[16] J. L. Bona, V. A. Dougalis, O. Karakashian, W. McKinney, and F. Smith. "Conservative, highorder numerical schemes for the generalized Korteweg—de Vries equation." In: Philosophical Transactions of the Royal Society of London. Series A: Physical and Engineering Sciences 351.1695 (1995), pp. 107-164. Dor: 10.1098/rsta.1995.0027.

[17] J. L. Bona, W. Pritchard, and L. R. Scott. "Numerical schemes for a model for nonlinear dispersive waves." In: Journal of Computational Physics 60.2 (1985), pp. 167-186.

[18] J. L. Bona, M. Chen, and J.-C. Saut. "Boussinesq equations and other systems for smallamplitude long waves in nonlinear dispersive media: II. The nonlinear theory." In: Nonlinearity 17.3 (2004), p. 925. DoI: 10.1088/0951-7715/17/3/010.

[19] J. Buli and Y. Xing. "Local discontinuous Galerkin methods for the Boussinesq coupled BBM systemalerkin methods for the Boussinesq coupled BBM system." In: Journal of Scientific Computing 75.1 (2018), pp. 536-559. Dor: 10.1007/s10915-017-0546-0.

[20] W. Cai, Y. Sun, and Y. Wang. "Geometric numerical integration for peakon $b$-family equations." In: Communications in Computational Physics 19.1 (2016), pp. 24-52. Dor: 10 . 4208 / cicp.171114.140715a.

[21] R. Camassa and D. D. Holm. "An integrable shallow water equation with peaked solitons." In: Physical Review Letters 71.11 (1993), p. 1661. Dor: 10.1103/PhysRevLett .71.1661.

[22] M. H. Carpenter and D. Gottlieb. "Spectral Methods on Arbitrary Grids." In: Journal of Computational Physics 129.1 (1996), pp. 74-86. Dor: 10.1006/jcph.1996.0234.

[23] M. H. Carpenter, D. Gottlieb, and S. Abarbanel. "Time-Stable Boundary Conditions for Finite-Difference Schemes Solving Hyperbolic Systems: Methodology and Application to High-Order Compact Schemes." In: Journal of Computational Physics 111.2 (1994), pp. 220236. Dor: $10.1006 / \mathrm{jcph} .1994 .1057$.

[24] M. H. Carpenter, J. Nordström, and D. Gottlieb. "A Stable and Conservative Interface Treatment of Arbitrary Spatial Accuracy." In: Journal of Computational Physics 148.2 (1999), pp. 341-365. Dor: 10.1006/jcph. 1998.6114.

[25] J. Chan. "On discretely entropy conservative and entropy stable discontinuous Galerkin methods." In: Journal of Computational Physics 362 (2018), pp. 346-374. Dor: 10 . 1016/j . jcp. 2018.02 .033 .

[26] J. Chan. "Skew-symmetric entropy stable modal discontinuous Galerkin formulations." In: Journal of Scientific Computing 81.1 (2019), pp. 459-485. Dor: 10. 1007/s10915-019-01026-w.

[27] J. Chan, D. C. D. R. Fernández, and M. H. Carpenter. "Efficient entropy stable Gauss collocation methods." In: SIAM Journal on Scientific Computing 41.5 (2019), A2938-A2966. DOI: $10.1137 / 18 \mathrm{M} 1209234$.

[28] T. Chen and C.-W. Shu. "Review of entropy stable discontinuous Galerkin methods for systems of conservation laws on unstructured simplex meshes." In: CSIAM Transactions on Applied Mathematics 1.1 (2020), pp. 1-52. Dor: 10.4208/csiam-am.2020-0003.

[29] B. Cockburn and C.-W. Shu. "The local discontinuous Galerkin method for time-dependent convection-diffusion systems." In: SIAM Journal on Numerical Analysis 35.6 (1998), pp. 24402463. DOI: $10.1137 /$ S0036142997316712.

[30] J De Frutos and J. M. Sanz-Serna. "Accuracy and conservation properties in numerical integration: the case of the Korteweg-de Vries equation." In: Numerische Mathematik 75.4 (1997), pp. 421-445. DoI: $10.1007 /$ s002110050247. 
[31] A. Degasperis, D. D. Holm, and A. N. Hone. "A new integrable equation with peakon solutions." In: Theoretical and Mathematical Physics 133.2 (2002), pp. 1463-1474. DOI: 10 . 1023/A: 1021186408422.

[32] K. Dekker and J. G. Verwer. Stability of Runge-Kutta methods for stiff nonlinear differential equations. Vol. 2. CWI Monographs. Amsterdam: North-Holland, 1984.

[33] A. Durán, D. Dutykh, and D. Mitsotakis. “On the multi-symplectic structure of Boussinesqtype systems. I: Derivation and mathematical properties." In: Physica D: Nonlinear Phenomena 388 (2019), pp. 10-21. Dor: 10.1016/j . physd.2018.11.007.

[34] A. Durán, D. Dutykh, and D. Mitsotakis. “On the multi-symplectic structure of Boussinesqtype systems. II: Geometric discretization." In: Physica D: Nonlinear Phenomena 397 (2019), pp. 1-16. Dor: $10.1016 / \mathrm{j}$. physd.2019.05.002.

[35] A. Durán and M. López-Marcos. "Conservative numerical methods for solitary wave interactions." In: Journal of Physics A: Mathematical and General 36.28 (2003), p. 7761. Dor: $10.1088 / 0305-4470 / 36 / 28 / 306$.

[36] A. Durán and J. Sanz-Serna. "The numerical integration of relative equilibrium solutions. The nonlinear Schrödinger equation." In: IMA Journal of Numerical Analysis 20.2 (2000), pp. 235-261. Dor: 10.1093/imanum/20.2.235.

[37] D. Dutykh, D. Clamond, P. Milewski, and D. Mitsotakis. "Finite volume and pseudospectral schemes for the fully nonlinear 1D Serre equations." In: European Journal of Applied Mathematics 24.5 (2013), pp. 761-787.

[38] D. Dutykh, T. Katsaounis, and D. Mitsotakis. "Finite volume methods for unidirectional dispersive wave models." In: International Journal for Numerical Methods in Fluids 71.6 (2013), pp. 717-736.

[39] J. Eilbeck and G. McGuire. "Numerical study of the regularized long-wave equation I: numerical methods." In: Journal of Computational Physics 19.1 (1975), pp. 43-57. Dor: 10 . 1016/0021-9991(75)90115-1.

[40] D. C. D. R. Fernández, P. D. Boom, M. H. Carpenter, and D. W. Zingg. “Extension of TensorProduct Generalized and Dense-Norm Summation-by-Parts Operators to Curvilinear Coordinates." In: Journal of Scientific Computing 80.3 (2019), pp. 1957-1996. Dor: 10 . 1007/s10915Q19-01011-3.

[41] D. C. D. R. Fernández, J. E. Hicken, and D. W. Zingg. "Review of summation-by-parts operators with simultaneous approximation terms for the numerical solution of partial differential equations." In: Computers E Fluids 95 (2014), pp. 171-196. Dor: 10 . 1016/ j . compfluid.2014.02.016.

[42] T. C. Fisher and M. H. Carpenter. "High-order entropy stable finite difference schemes for nonlinear conservation laws: Finite domains." In: Journal of Computational Physics 252 (2013), pp. 518-557. Dor: 10.1016/j . jcp.2013.06.014.

[43] T. C. Fisher, M. H. Carpenter, J. Nordström, N. K. Yamaleev, and C. Swanson. “Discretely conservative finite-difference formulations for nonlinear conservation laws in split form: Theory and boundary conditions." In: Journal of Computational Physics 234 (2013), pp. 353375. DOI: $10.1016 / \mathrm{j}$. jcp.2012.09.026.

[44] B. Fornberg. "On a Fourier method for the integration of hyperbolic equations." In: SIAM Journal on Numerical Analysis 12.4 (1975), pp. 509-528. Dor: 10.1137/0712040.

[45] G. J. Gassner. "A Skew-Symmetric Discontinuous Galerkin Spectral Element Discretization and Its Relation to SBP-SAT Finite Difference Methods." In: SIAM Journal on Scientific Computing 35.3 (2013), A1233-A1253. Dor: 10.1137/120890144. 
[46] G. J. Gassner, A. R. Winters, and D. A. Kopriva. "Split Form Nodal Discontinuous Galerkin Schemes with Summation-By-Parts Property for the Compressible Euler Equations." In: Journal of Computational Physics 327 (2016), pp. 39-66. Dor: 10.1016/j . jcp. 2016.09.013.

[47] J. E. Hicken. "Entropy-stable, high-order summation-by-parts discretizations without interface penalties." In: Journal of Scientific Computing 82.2 (2020), p. 50. Dor: 10 . 1007/s10915020-01154-8.

[48] J. E. Hicken, D. C. D. R. Fernández, and D. W. Zingg. "Multidimensional Summation-ByParts Operators: General Theory and Application to Simplex Elements." In: SIAM Journal on Scientific Computing 38.4 (2016), A1935-A1958. Dor: 10.1137/15M1038360.

[49] D. D. Holm and A. N. Hone. "Nonintegrability of a fifth-order equation with integrable two-body dynamics." In: Theoretical and Mathematical Physics 137.1 (2003), pp. 1459-1471. DOI: 10.1023/A: 1026060924520.

[50] Q. Hong, Y. Gong, and Z. Lv. "Linear and Hamiltonian-conserving Fourier pseudo-spectral schemes for the Camassa-Holm equation." In: Applied Mathematics and Computation 346 (2019), pp. 86-95. Dor: 10.1016/j . amc .2018.10.043.

[51] J. D. Hunter. "Matplotlib: A 2D graphics environment." In: Computing in Science \& Engineering 9.3 (2007), pp. 90-95. Dor: 10.1109/MCSE . 2007. 55.

[52] L. Jameson. "On the wavelet based differentiation matrix." In: Journal of Scientific Computing 8.3 (1993), pp. 267-305. DOI: 10.1007/BF01060934.

[53] D. I. Ketcheson. "Relaxation Runge-Kutta Methods: Conservation and Stability for InnerProduct Norms." In: SIAM Journal on Numerical Analysis 57.6 (2019), pp. 2850-2870. Dor: 10.1137/19M1263662. arXiv: 1905.09847 [math.NA].

[54] S. Koide and D. Furihata. "Nonlinear and linear conservative finite difference schemes for regularized long wave equation." In: Japan Journal of Industrial and Applied Mathematics 26.1 (2009), p. 15. DOI: $10.1007 /$ BFO3167544.

[55] D. A. Kopriva. Implementing Spectral Methods for Partial Differential Equations: Algorithms for Scientists and Engineers. New York: Springer Science \& Business Media, 2009. Dor: 10.1007/ 978-90-481-2261-5.

[56] D. A. Kopriva and G. J. Gassner. “On the Quadrature and Weak Form Choices in Collocation Type Discontinuous Galerkin Spectral Element Methods." In: Journal of Scientific Computing 44.2 (2010), pp. 136-155. Dor: 10.1007/s10915-010-9372-3.

[57] H.-O. Kreiss and J. Oliger. "Comparison of accurate methods for the integration of hyperbolic equations." In: Tellus 24.3 (1972), pp. 199-215. Dor: 10.3402/tellusa.v24i3 . 10634.

[58] H.-O. Kreiss and G. Scherer. "Finite Element and Finite Difference Methods for Hyperbolic Partial Differential Equations." In: Mathematical Aspects of Finite Elements in Partial Differential Equations. Ed. by C. de Boor. New York: Academic Press, 1974, pp. 195-212.

[59] W. Kutta. "Beitrag zur näherungsweisen Integration totaler Differentialgleichungen." In: Zeitschrift für Mathematik und Physik 46 (1901), pp. 435-453.

[60] P. G. LeFloch, J.-M. Mercier, and C. Rohde. "Fully Discrete, Entropy Conservative Schemes of Arbitrary Order." In: SIAM Journal on Numerical Analysis 40.5 (2002), pp. 1968-1992. Dor: 10.1137/S003614290240069X.

[61] R. J. LeVeque. Finite Difference Methods for Ordinary and Partial Differential Equations. SteadyState and Time-Dependent Problems. Philadelphia: Society for Industrial and Applied Mathematics, 2007. DoI: 10.1137/1.9780898717839.

[62] X. Li, Y. Xing, and C.-S. Chou. “Optimal Energy Conserving and Energy Dissipative Local Discontinuous Galerkin Methods for the Benjamin-Bona-Mahony Equation." In: Journal of Scientific Computing 83.17 (2020). Dor: 10.1007/s10915-020-01172-6. 
[63] H. Liu and T. Pendleton. "On Invariant-Preserving Finite Difference Schemes for the Camassa-Holm Equation and the Two-Component Camassa-Holm System." In: Communications in Computational Physics 19.4 (2016), pp. 1015-1041. Dor: 10 . 4208/cicp . 130115. $110915 \mathrm{a}$.

[64] H. Liu and Y. Xing. "An Invariant Preserving Discontinuous Galerkin Method for the Camassa-Holm Equation." In: SIAM Journal on Scientific Computing 38.4 (2016), A1919A1934. DOI: 10.1137/15M102705X.

[65] K. Mattsson. "Diagonal-norm summation by parts operators for finite difference approximations of third and fourth derivatives." In: Journal of Computational Physics 274 (2014), pp. 432-454. Dor: 10.1016/j . jcp.2014.06.027.

[66] K. Mattsson. "Diagonal-norm upwind SBP operators." In: Journal of Computational Physics 335 (2017), pp. 283-310. Dor: 10.1016/j . jcp.2017.01.042.

[67] K. Mattsson, M. Almquist, and M. H. Carpenter. "Optimal diagonal-norm SBP operators." In: Journal of Computational Physics 264 (2014), pp. 91-111. Dor: 10.1016/j . j cp . 2013 . 12 . 041.

[68] K. Mattsson, M. Almquist, and E. van der Weide. "Boundary optimized diagonal-norm SBP operators." In: Journal of Computational Physics 374 (2018), pp. 1261-1266. DOI: 10 . 1016/j . jсp. 2018.06.010.

[69] K. Mattsson and J. Nordström. "Summation by parts operators for finite difference approximations of second derivatives." In: Journal of Computational Physics 199.2 (2004), pp. 503-540. DOI: $10.1016 / \mathrm{j} . \mathrm{jcp} .2004 .03 .001$.

[70] K. Mattsson and O. O'Reilly. "Compatible diagonal-norm staggered and upwind SBP operators." In: Journal of Computational Physics 352 (2018), pp. 52-75. Dor: 10 . 1016/j . jcp . 2017. 09.044.

[71] K. Mattsson, M. Svärd, and M. Shoeybi. "Stable and accurate schemes for the compressible Navier-Stokes equations." In: Journal of Computational Physics 227.4 (2008), pp. 2293-2316. DOI: $10.1016 / \mathrm{j} \cdot \mathrm{jcp} .2007 \cdot 10.018$.

[72] J. Nordström. “Conservative Finite Difference Formulations, Variable Coefficients, Energy Estimates and Artificial Dissipation." In: Journal of Scientific Computing 29.3 (2006), pp. 375404. DOI: $10.1007 / \mathrm{s} 10915-005-9013-4$.

[73] J. Nordström and M. Björck. "Finite volume approximations and strict stability for hyperbolic problems." In: Applied Numerical Mathematics 38.3 (2001), pp. 237-255. Dor: 10 . 1016/ SQ168-9274(01)00027-7.

[74] J. Nordström, K. Forsberg, C. Adamsson, and P. Eliasson. "Finite volume methods, unstructured meshes and strict stability for hyperbolic problems." In: Applied Numerical Mathematics 45.4 (2003), pp. 453-473. DOI: 10.1016/S0168-9274(02)00239-8.

[75] P. J. Olver. "Euler operators and conservation laws of the BBM equation." In: Mathematical Proceedings of the Cambridge Philosophical Society. Vol. 85. 1. Cambridge University Press. 1979, pp. 143-160. Dor: 10.1017/S0305004100055572.

[76] B. Pelloni and V. A. Dougalis. "Numerical modelling of two-way propagation of non-linear dispersive waves." In: Mathematics and computers in simulation 55.4-6 (2001), pp. 595-606.

[77] V. Petviashvili. "Equation of an extraordinary soliton (ion acoustic wave packet dispersion in plasma)." In: Soviet Journal of Plasma Physics 2 (1976), p. 257.

[78] C. Rackauckas and Q. Nie. "DifferentialEquations.jl - A Performant and Feature-Rich Ecosystem for Solving Differential Equations in Julia." In: Journal of Open Research Software 5.1 (2017), p. 15. Dor: 10.5334/jors. 151.

[79] H. Ranocha. "Comparison of Some Entropy Conservative Numerical Fluxes for the Euler Equations." In: Journal of Scientific Computing 76.1 (July 2018), pp. 216-242. Dor: 10 . 1007 / s10915-017-0618-1. arXiv: 1701.02264 [math.NA]. 
[80] H. Ranocha. "Generalised Summation-by-Parts Operators and Entropy Stability of Numerical Methods for Hyperbolic Balance Laws." PhD thesis. TU Braunschweig, Feb. 2018.

[81] H. Ranocha. "Generalised Summation-by-Parts Operators and Variable Coefficients." In: Journal of Computational Physics 362 (Feb. 2018), pp. 20-48. Dor: 10 . 1016/j . jcp . 2018 . 02 . Q21. arXiv: 1705.10541 [math.NA].

[82] H. Ranocha. "Mimetic Properties of Difference Operators: Product and Chain Rules as for Functions of Bounded Variation and Entropy Stability of Second Derivatives." In: BIT Numerical Mathematics 59.2 (June 2019), pp. 547-563. Dor: 10 . 1007/s10543-018-0736-7. arXiv: 1805.09126 [math.NA].

[83] H. Ranocha, L. Dalcin, and M. Parsani. "Fully-Discrete Explicit Locally Entropy-Stable Schemes for the Compressible Euler and Navier-Stokes Equations." In: Computers and Mathematics with Applications 80.5 (July 2020), pp. 1343-1359. Dor: 10.1016/j . camwa. 2020.06.016. arXiv: 2003.08831 [math.NA].

[84] H. Ranocha and D. I. Ketcheson. "Relaxation Runge-Kutta Methods for Hamiltonian Problems." In: Journal of Scientific Computing 84.1 (July 2020). Dor: 10. 1007/s10915-020-01277-y. arXiv: 2001.04826 [math.NA].

[85] H. Ranocha, L. Lóczi, and D. I. Ketcheson. General Relaxation Methods for Initial-Value Problems with Application to Multistep Schemes. Mar. 2020. arXiv: 2003.03012 [math.NA].

[86] H. Ranocha, D. Mitsotakis, and D. I. Ketcheson. Dispersive-wave-schemes-notebooks. A Broad Class of Conservative Numerical Methods for Dispersive Wave Equations. https : / / github . com/ranocha/Dispersive-wave-schemes-notebooks. June 2020. Dor: 10 . 5281/zenodo. 3908803.

[87] H. Ranocha, P. Öffner, and T. Sonar. "Extended skew-symmetric form for summation-byparts operators and varying Jacobians." In: Journal of Computational Physics 342 (Apr. 2017), pp. 13-28. Dor: 10.1016/j.jcp.2017.04.044. arXiv: 1511.08408 [math.NA].

[88] H. Ranocha, P. Öffner, and T. Sonar. "Summation-by-parts operators for correction procedure via reconstruction." In: Journal of Computational Physics 311 (Apr. 2016), pp. 299-328. DOI: 10.1016/j.jcp.2016.02.009. arXiv: 1511.02052 [math.NA].

[89] H. Ranocha, M. Sayyari, L. Dalcin, M. Parsani, and D. I. Ketcheson. "Relaxation RungeKutta Methods: Fully-Discrete Explicit Entropy-Stable Schemes for the Compressible Euler and Navier-Stokes Equations." In: SIAM Journal on Scientific Computing 42.2 (Mar. 2020), A612-A638. DoI: 10.1137/19M1263480. arXiv: 1905.09129 [math.NA].

[90] R. D. Richtmyer and K. W. Morton. Difference Methods for Boundary-Value Problems. New York, London, Sydney: John Wiley \& Sons, 1967.

[91] P. J. Roache. "Code verification by the method of manufactured solutions." In: Journal of Fluids Engineering 124.1 (2002), pp. 4-10. DoI: 10.1115/1.1436090.

[92] J. M. Sanz-Serna. "An explicit finite-difference scheme with exact conservation properties." In: Journal of Computational Physics 47.2 (1982), pp. 199-210. Dor: 10 . 1016/0021-9991 (82) 90074-2.

[93] J. M. Sanz-Serna and V. Manoranjan. "A method for the integration in time of certain partial differential equations." In: Journal of Computational Physics 52.2 (1983), pp. 273-289. DOr: 10.1016/0021-9991(83)90031-1.

[94] B. Strand. "Summation by Parts for Finite Difference Approximations for $d / d x$." In: Journal of Computational Physics 110.1 (1994), pp. 47-67. Dor: 10.1006/jcph. 1994.1005.

[95] M. Svärd and J. Nordström. "Review of summation-by-parts schemes for initial-boundaryvalue problems." In: Journal of Computational Physics 268 (2014), pp. 17-38. Dor: 10. 1016/j . jcp.2014.02.031. 
[96] E. Tadmor. "The numerical viscosity of entropy stable schemes for systems of conservation laws. I." In: Mathematics of Computation 49.179 (1987), pp. 91-103. DOr: 10 . 1090 / S00255718-1987-0890255-3.

[97] C. Tsitouras. "Runge-Kutta pairs of order 5 (4) satisfying only the first column simplifying assumption." In: Computers $\mathcal{E}$ Mathematics with Applications 62.2 (2011), pp. 770-775. Dor: $10.1016 / \mathrm{j}$. camwa. 2011.06 .002 .

[98] J. H. Verner. “Numerically optimal Runge-Kutta pairs with interpolants." In: Numerical Algorithms 53.2-3 (2010), pp. 383-396. Dor: 10.1007/s11075-009-9290-3.

[99] M Walkley and M. Berzins. "A finite element method for the one-dimensional extended Boussinesq equations." In: International Journal for Numerical Methods in Fluids 29.2 (1999), pp. 143-157. DOI: 10 . 1002 / (SICI) 1097-0363(19990130) 29: 2<143 : : AID-FLD779>3 . 0 . CO;2-5.

[100] G. Wei, J. T. Kirby, S. T. Grilli, and R. Subramanya. "A fully nonlinear Boussinesq model for surface waves. Part 1. Highly nonlinear unsteady waves." In: Journal of Fluid Mechanics 294 (1995), 71-92. DOI: 10.1017/S0022112095002813.

[101] G. B. Whitham. "Variational methods and applications to water waves." In: Proceedings of the Royal Society of London. Series A. Mathematical and Physical Sciences 299.1456 (1967), pp. 6-25. DOI: $10.1098 / \mathrm{rspa} .1967 .0119$.

[102] A. R. Winters, R. C. Moura, G. Mengaldo, G. J. Gassner, S. Walch, J. Peiro, and S. J. Sherwin. "A comparative study on polynomial dealiasing and split form discontinuous Galerkin schemes for under-resolved turbulence computations." In: Journal of Computational Physics 372 (2018), pp. 1-21. DoI: 10.1016/j . jcp.2018.06.016.

[103] R. Winther. "A conservative finite element method for the Korteweg-de Vries equation." In: Mathematics of computation (1980), pp. 23-43. Dor: 10.2307/2006219.

[104] R. Winther. "A finite element method for a version of the Boussinesq equation." In: SIAM Journal on Numerical Analysis 19.3 (1982), pp. 561-570. Dor: 10.1137/0719037.

[105] Y. Xia. "Fourier spectral methods for Degasperis-Procesi equation with discontinuous solutions." In: Journal of Scientific Computing 61.3 (2014), pp. 584-603. Dor: 10. 1007/s10915Q14-9839-8.

[106] Y. Xu and C.-W. Shu. "A local discontinuous Galerkin method for the Camassa-Holm equation." In: SIAM Journal on Numerical Analysis 46.4 (2008), pp. 1998-2021. Dor: 10.1137/ 070679764.

[107] Y.Xu and C.-W.Shu. "Local discontinuous Galerkin methods for high-order time-dependent partial differential equations." In: Communications in Computational Physics 7.1 (2010), pp. 146. DOI: $10.4208 /$ cicp.2009.09.023.

[108] Y. Xu and C.-W. Shu. "Local discontinuous Galerkin methods for the Degasperis-Procesi equation." In: Communications in Computational Physics 10.2 (2011), pp. 474-508. Dor: 10. 4208/cicp. 300410.300710 a.

[109] J. Yan and C.-W. Shu. "A local discontinuous Galerkin method for KdV type equations." In: SIAM Journal on Numerical Analysis 40.2 (2002), pp. 769-791.

[110] Y. Yang, Y. Wang, and Y. Song. "A new local energy-preserving algorithm for the BBM equation." In: Applied Mathematics and Computation 324 (2018), pp. 119-130. Dor: 10 . 1016/ j . amc. 2017.12 .013$.

[111] Q. Zhang, Y. Xu, and C.-W. Shu. Dissipative and conservative local discontinuous Galerkin methods for the Fornberg-Whitham type equations. 2020. uRL: https: //www . brown. edu/research/ projects/scientific-computing/sites/brown. edu.research.projects. scientificcomputing/files/uploads/Dissipative\%20and\%20conservative\%201ocal\%20discontinuous . pdf. 
[112] H. Zhu, S. Song, and Y. Tang. "Multi-symplectic wavelet collocation method for the nonlinear Schrödinger equation and the Camassa-Holm equation." In: Computer Physics Communications 182.3 (2011), pp. 616-627. DOI: 10.1016/j .cpc.2010.11.003. 